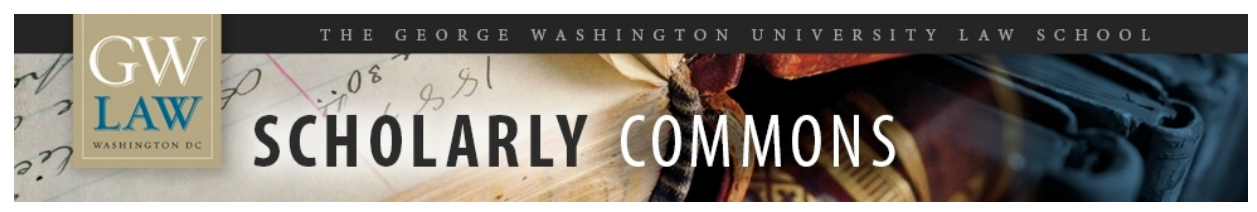

GW Law Faculty Publications \& Other Works

Faculty Scholarship

2011

\title{
Constitutional Law: A Contemporary Approach
}

Gregory E. Maggs

George Washington University Law School, gmaggs@law.gwu.edu

Peter J. Smith

George Washington University Law School, pjsmith@law.gwu.edu

Follow this and additional works at: https://scholarship.law.gwu.edu/faculty_publications

Part of the Law Commons

\section{Recommended Citation}

Gregory E. Maggs \& Peter J. Smith, Constitutional Law: A Contemporary Approach (2d ed. 2011).

This Book Part is brought to you for free and open access by the Faculty Scholarship at Scholarly Commons. It has been accepted for inclusion in GW Law Faculty Publications \& Other Works by an authorized administrator of Scholarly Commons. For more information, please contact spagel@law.gwu.edu. 


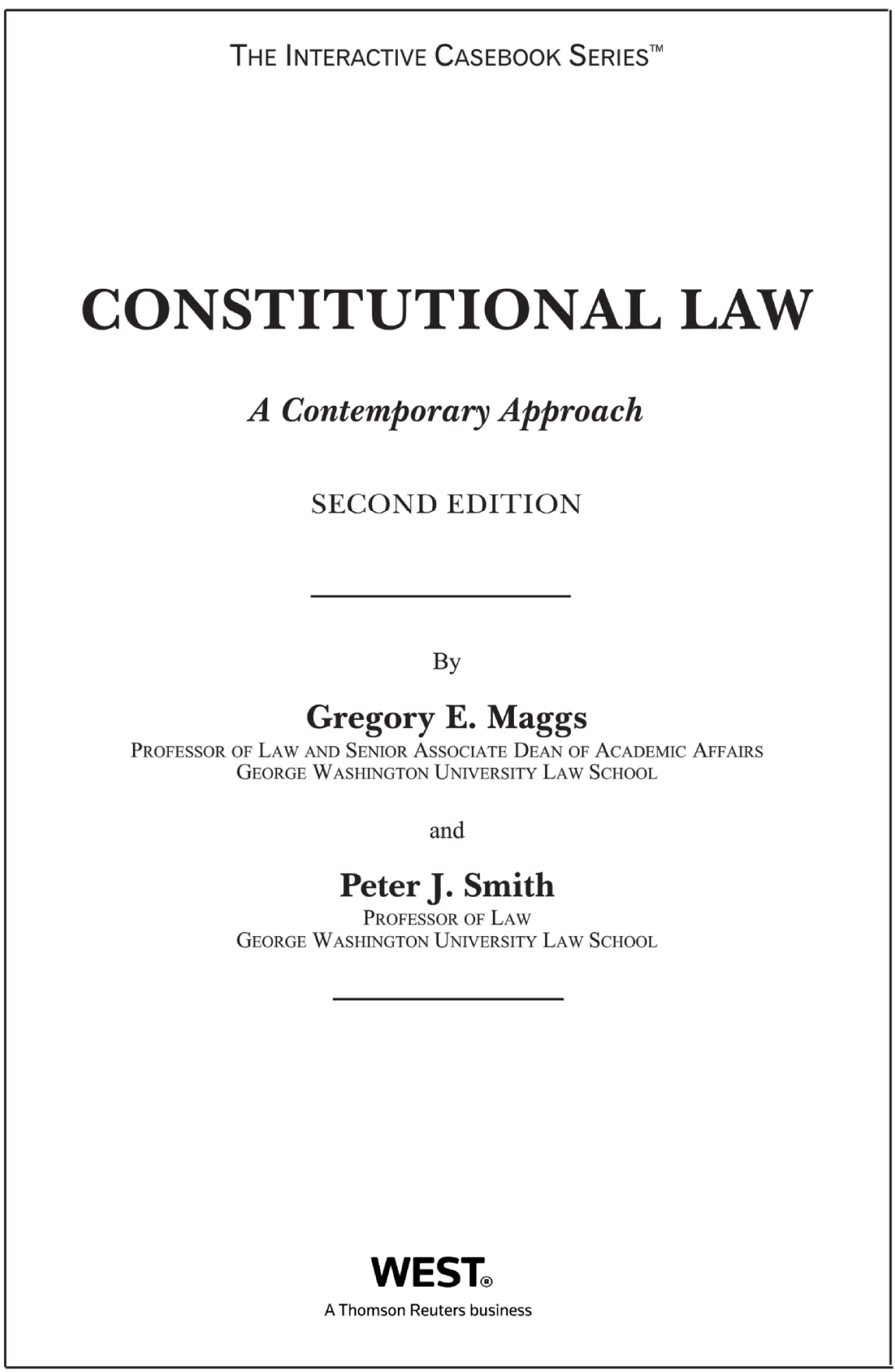

Mat \#41105530 
Thomson Reuters created this publication to provide you with accurate and authoritative information concerning the subject matter covered. However, this publication was not necessarily prepared by persons licensed to practice law in a particular jurisdiction. Thomson Reuters does not render legal or other professional advice, and this publication is not a substitute for the advice of an attorney. If you require legal or other expert advice, you should seek the services of a competent attorney or other professional.

Interactive Casebook Series is a trademark registered in the U.S. Patent and Trademark Office.

(C) 2009 Thomson Reuters

(C) 2011 Thomson Reuters

610 Opperman Drive

St. Paul, MN 55123

$1-800-313-9378$

Printed in the United States of America

ISBN: 978-0-314-27355-0 


\section{Preface}

American Constitutional Law is a sprawling subject. Some law schools cover the topic in one course, others in two or three; some cover it in the first year, others only in the second and third years, and still others teach some of it in the first year and then more in the final two years. The subject is so large that most law schools also teach pieces of it in classes principally focused on Administrative Law, Criminal Procedure, Civil Procedure, Property, and many other topics.

American legal publishers have produced scores of casebooks to fit this range of approaches. It was thus with some trepidation that we decided to add our book to the mix. In the end, we concluded that we had a contribution to make, even to this rich market. There is, of course, something like a "canon" in constitutional law, which means that a book is unlikely to be distinctive for the cases that it chooses to cover; a constitutional law casebook would not be particularly useful, for example, if it did not include Marbury v. Madison, 5 U.S. (1 Cranch) 137 (1803), McCulloch v. Maryland, 17 U.S. (4 Wheat.) 316 (1819), Brown v. Board of Education of Topeka, 347 U.S. 483, 489 (1954), or many others. Instead, we think our book is distinctive-and thus will be especially effective in teaching the subject-for four principal reasons.

First, the book comes with an electronic version that students can access on their computers. The electronic version contains live hyperlinks to cases, statutes, law review articles, and other materials available on Westlaw and other websites. Accordingly, students who wish to explore the material in greater depth have the tools at their fingertips to do so. In addition, students who use laptop computers in the classroom will be able to view the course materials on their computers in class.

Second, we have selected the principal cases very carefully, and we have tried to avoid the problem created by the aggressive editing in many books, which present excerpts so brief that the students in effect read only about what the Supreme Court has decided. But Supreme Court opinions should be presented as more than a series of conclusory assertions that have been stitched together by a space-sensitive editor. We have tried to edit the principal cases to ensure that they are short enough to read, but rich enough to give the students a clear sense of the Court's reasoning. We have also chosen not to create the illusion of breadth that characterizes many books in the field. Rather than provide summaries of dozens of decisions in each area that we take up, we focus on fuller excerpts of the principal cases, which are designed to be illustrative. Our book is self-consciously a casebook, and does not aspire to be a treatise.

Third, rather than follow the principal cases with pages and pages of notes and questions-an ineffective approach that students universally resent-we include multiple 
sidebars in the excerpts of each principal case to focus the students' attention on important questions at the very moment when they are reading the relevant portions of the opinion. Among other things, the sidebars focus attention on particularly salient passages of the opinions; draw connections between the discussion in the case and other topics that the students have explored (or will explore) in the book; supply food for thought; and direct the students to secondary materials to enrich their studies. After each case, we provide brief points for discussion, to focus the students' attention on the central themes in the case. Each Chapter also contains hypothetical problems-often drawn from real cases-to encourage the students to apply the doctrine that they have learned, and ends with an "executive summary" of the material, to identify the main themes and doctrines covered in the Chapter.

Fourth, throughout the book we include "Point-Counterpoints," in which we provide arguments for and against central questions raised by the materials in the book. To be sure, throughout the book-in the points for discussion and in short excerpts from scholarly articles by leading experts in the field-we present a diversity of views on every subject. But the Point-Counterpoint discussions are presented in our own voices and reflect our genuine points of disagreement on the many disputed questions raised in the book. We think that the students will find these discussions rich and stimulating.

The first edition of the book covered decisions through the Supreme Court's October 2007 term, which ended in June 2008. The second edition expands the excerpts of many of the cases and includes cases decided since 2008. The principal cases range from the old chestnuts to significant cases of much more recent vintage; from (to take just a few examples) Barron v. Baltimore, 32 U.S. (7 Pet.) 243 (1833), to McDonald v. City of Chicago, 130 S.Ct. 3020 (2010); from Youngstown Sheet \& Tube Co. v. Sawyer, 343 U.S. 579 (1952), to Hamdi v. Rumsfeld, 542 U.S. 507 (2004); from Dred Scott v. Sandford, 60 U.S. 393 (1857), to Parents Involved in Comm. Schools v. Seattle Sch. Dist. No. 1, 127 S. Ct. 2738 (2007). In addition, Part I, which provides background and an overview, ends with District of Columbia v. Heller, 554 U.S. 570 (2008), the recent Second Amendment decision, as an illustrative case to foreshadow the themes that will recur in the rest of the book.

Although we have attempted to provide fuller excerpts of the principal cases than is perhaps common in casebooks in the field, we of course nevertheless have had to do substantial editing. We have used three asterisks to indicate that text has been omitted within a paragraph, although we have often omitted entire paragraphs without providing a similar indication. (Because the cases are hyperlinked, students can easily read the full opinions, if they so choose.) We have omitted most footnotes from the cases; when we have included them, we have used the original numbering from the cases. Footnotes that we have inserted in the cases, on the other hand, are indicated by an asterisk and conclude with the notation "-Eds." We have also omitted many of the citations, but we have attempted to preserve the most important ones, which are also hyperlinked for the convenience of the students. 
We wish to acknowledge the many people who have helped us to produce this book. Many people helped us with the first edition. Our colleagues Tom Colby, Chip Lupu, and Robert Tuttle gave helpful suggestions, both for organization and content. Jonathan Affleck, Julia Carrano, Bob Chen, Courtney Enlow, and Brad Sauer went above and beyond as research assistants, editing cases and offering insightful commentary. Holly Saari did a terrific job making a dry manuscript come alive on the page. Louis Higgins graciously invited us to write the book and provided constant guidance and aid in seeing it through to completion. Dean Fred Lawrence and the George Washington University Law School also offered generous support.

We have had a similar embarrassment of riches with the second edition. Brad Clark, Tom Colby, Chip Lupu, Jeff Powell, and David Stras gave us invaluable feedback on the first edition (which they were kind enough to use in their classes). James Brower, Jamie Goldman, Elizabeth Gray, Ann Hodulik, Brittany Honeyman, Lindsay Lane, Richard Martinelli, Kyle Noonan, and Corrina Sowden provided outstanding research assistance. And Megan Anderson and Holly Saari again transformed our messy mass of edits into a clean and attractive book.

Gregory Maggs dedicates his contributions to the book to his patient family and to the many generations of law students at the George Washington University who have contributed to his understanding of constitutional law. Peter Smith dedicates his contributions to the book to Eileen and David Smith and Laurie Sparling, who have always provided unconditional love and support, and to Jonah and Sarah, whose already highly refined sense of justice has consistently challenged his own.

We hope that reading this book is as enjoyable for you as writing it was for us.

Gregory E. Maggs

Peter J. Smith

March 2011

Washington, DC 


\section{Table of Contents}

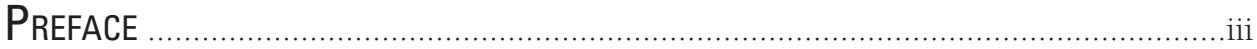

TABLE OF CONTENTS ......................................................................................... vii

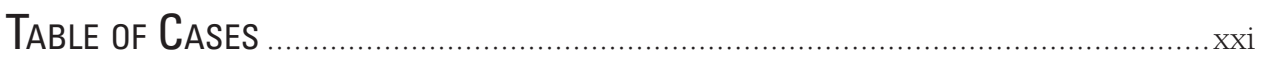

PART I: INTRODUCTION ................................................................................

ChaPTER 1 History and Overview ...........................................................

A. Historical Setting of the Constitution ................................................................

1. Colonial Governance and Events Preceding the Revolution ............................... 2

2. The First and Second Continental Congresses ............................................... 3

3. The Articles of Confederation and the Caluing of a Constitutional

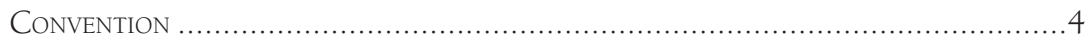

4. Proceedings of the Constitutional Convention …...................................... 5

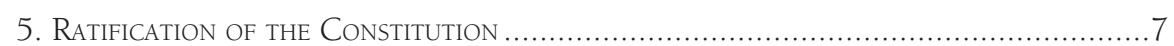

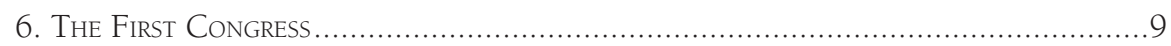

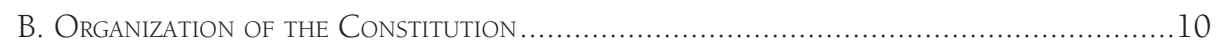

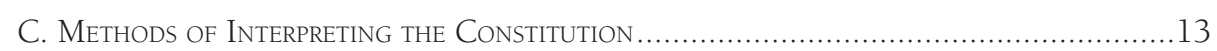

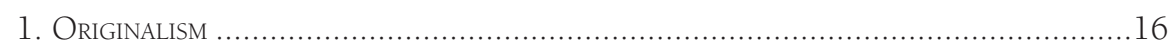

Perspectives and Analysis: Justice Antonin Scalia .......................................... 18

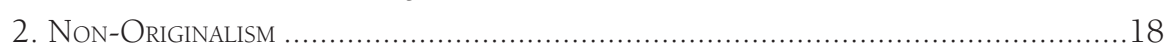

Perspectives and Analysis: Justice William Brennan, Jr. .................................21

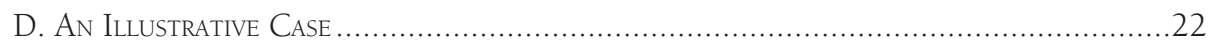

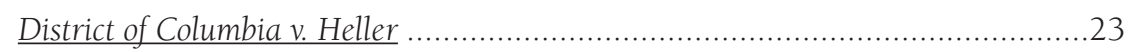




\section{Point-Counterpoint}

Does Heller represent originalism's triumph or its failure?

Part II: The Federal Courts

ChAPTER 2 Judicial Power.

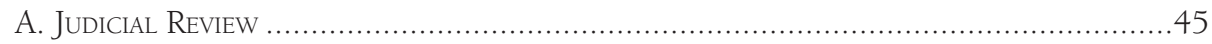

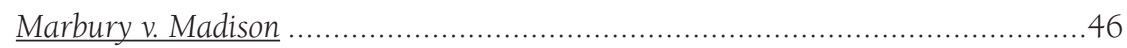

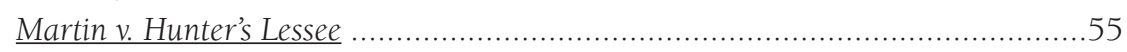

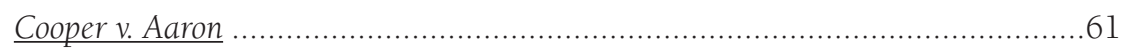

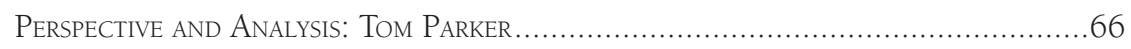

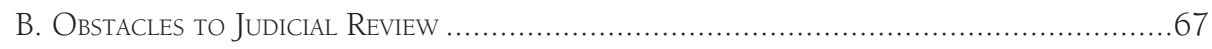

1. The Political Question Doctrine ......................................................................67

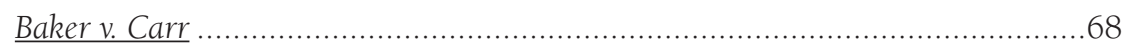

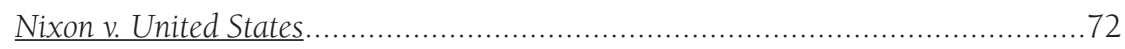

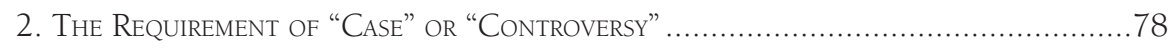

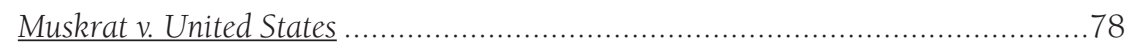

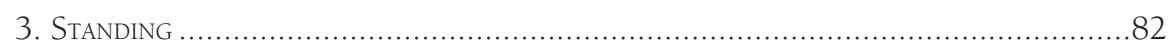

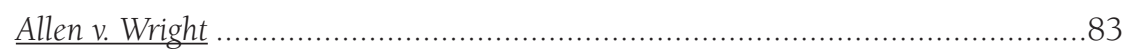

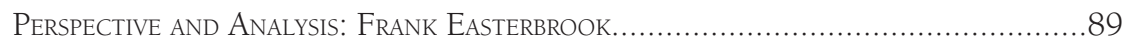

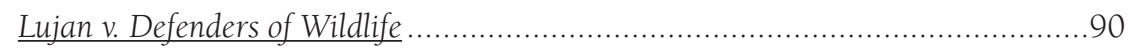

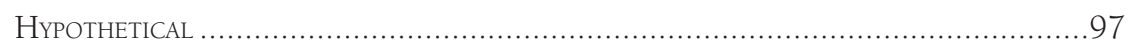

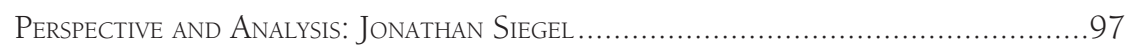

4. Congressional Control Over Federal Court Jurisdiction ...................................98

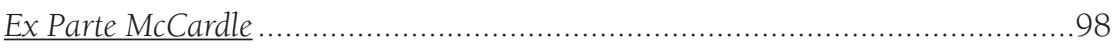

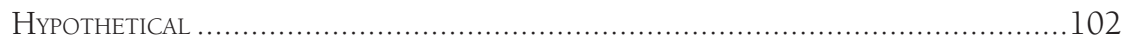

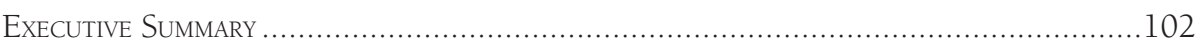

\section{Point-Counterpoint}

What is the role of the federal courts in our constitutional scheme? ...........................102

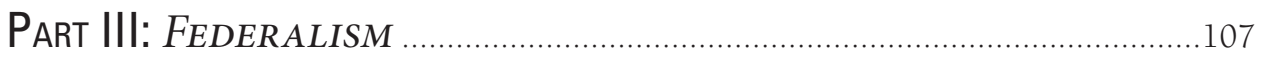

Chapter 3 Federal Legislative Power....................................................109

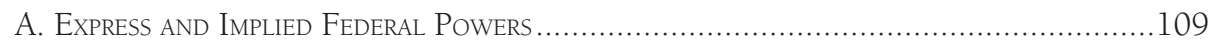

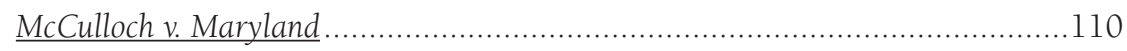

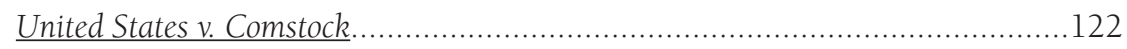




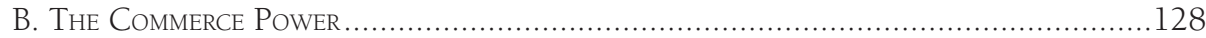

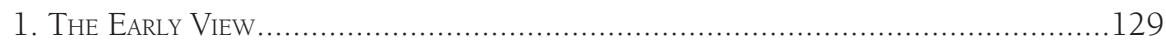

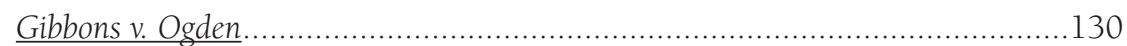

2. The Middle Years: Uncertainty and a Restrictive View..................................134

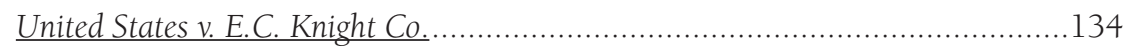

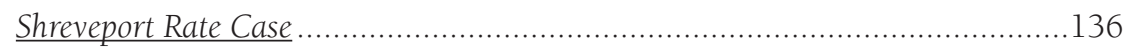

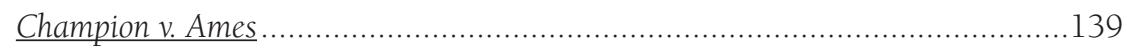

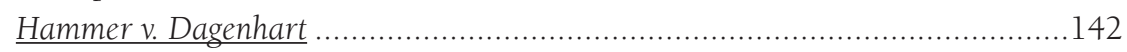

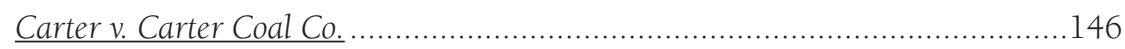

3. The New Deal and Beyond: An Expansive View …...........................................149

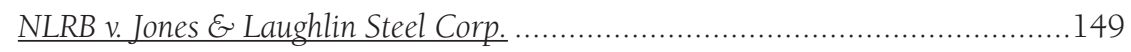

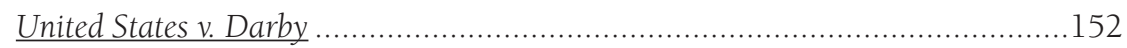

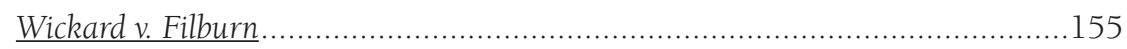

Heart of Atlanta Motel, Inc. v. United States ......................................................158

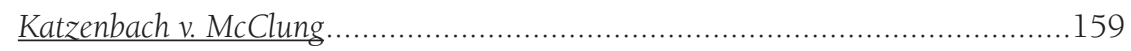

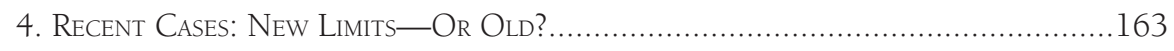

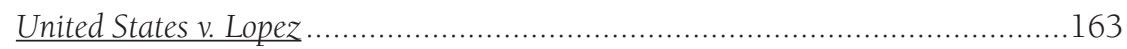

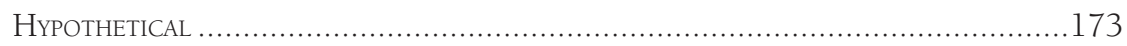

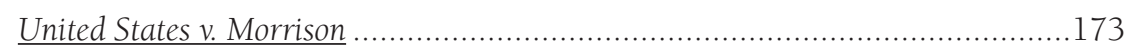

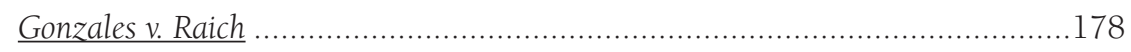

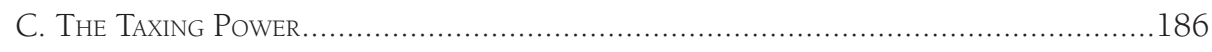

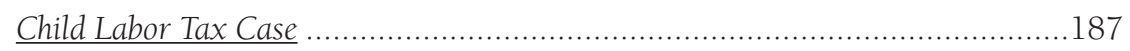

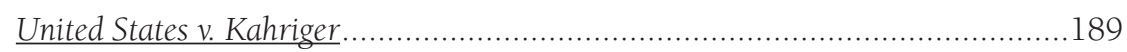

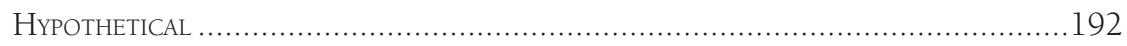

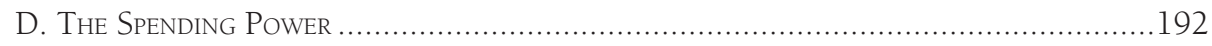

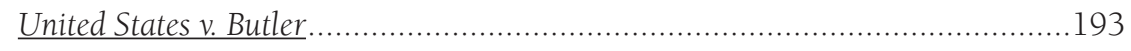

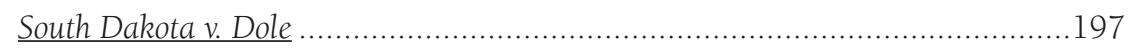

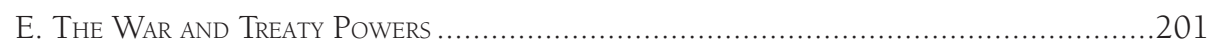

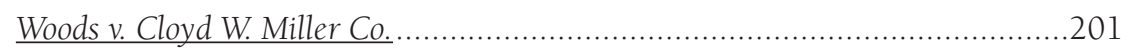

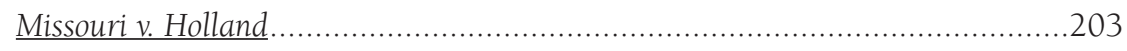

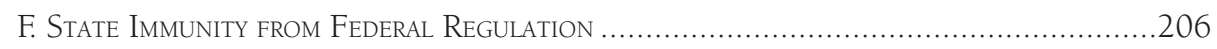

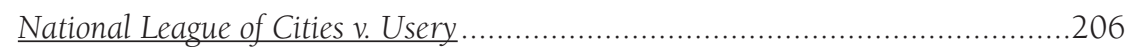

Garcia v. San Antonio Metropolitan Transit Authority ......................................209

New York v. United States ...........................................................................213

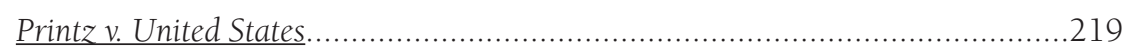

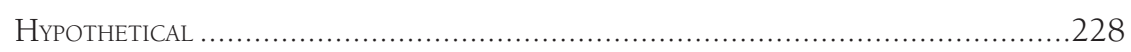

G. State Sovereign Immunity as a Limit on Federal Power …..................................228

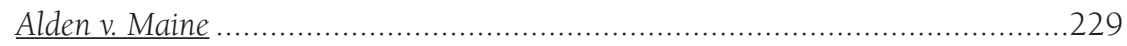

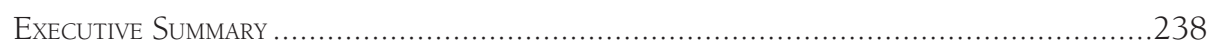




\section{Point-Counterpoint}

Was the expansion of federal powers inevitable? Is it defensible?

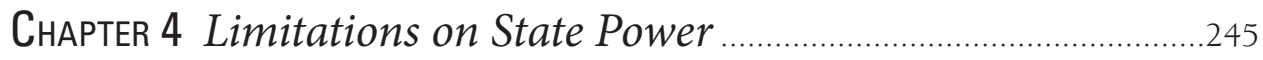

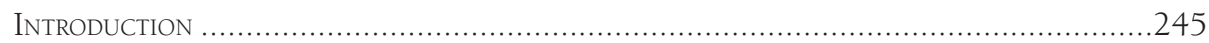

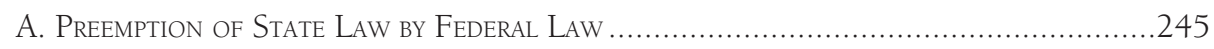

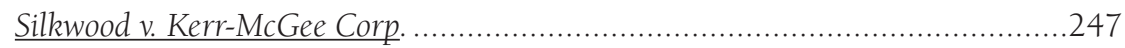

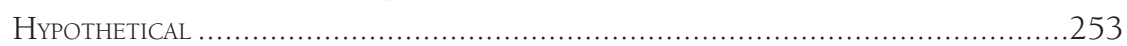

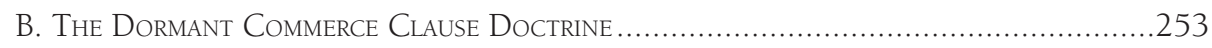

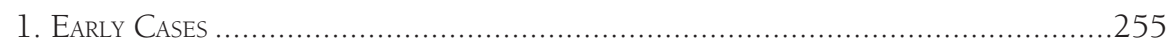

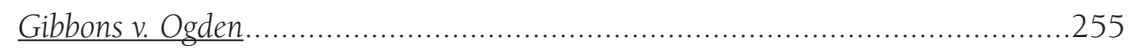

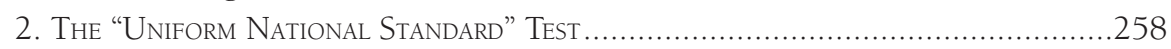

Cooley v. Board of Wardens .........................................................................25

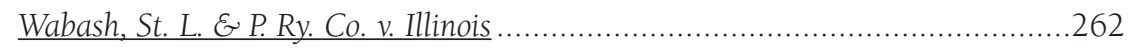

3. The "Discrimination Against Interstate Commerce" Test .................................264

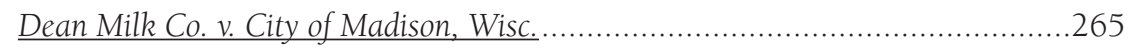

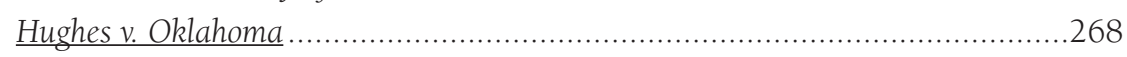

4. The "Excessive Burden on Interstate Commerce" TeSt ......................................22

South Carolina State Highway Dept. v. Barnwell Bros. .....................................22

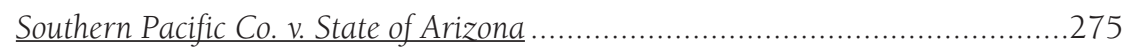

Kassel v. Consolidated Freightways Corp. of Delaware ……..............................279

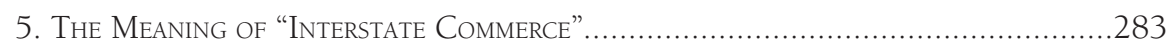

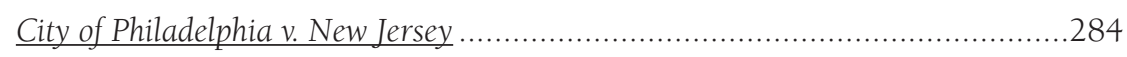

Camps Newfound/Owatonna, Inc. v. Town of Harrison ……............................28

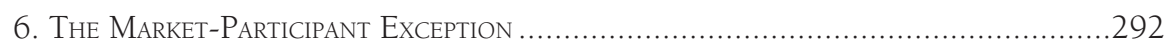

South-Central Timber Development, Inc. v. Wunnicke.....................................292

United Haulers Ass'n, Inc. v. Oneida-Herkimer Solid Waste Management Auth. ...297

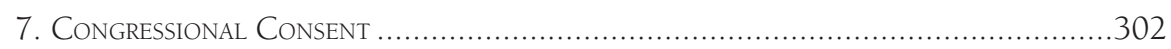

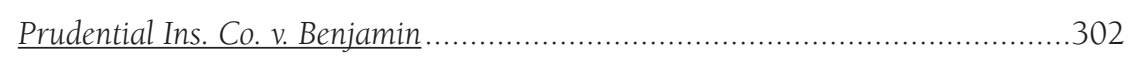

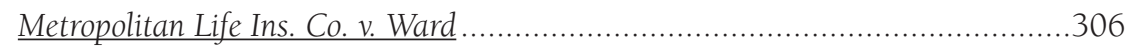

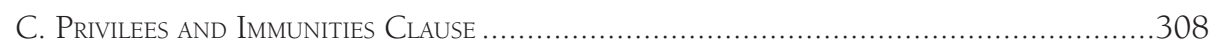

Baldwin v. Fish and Game Commission of Montana .........................................310

D. State Power to Regulate the House and Senate ................................................ 313

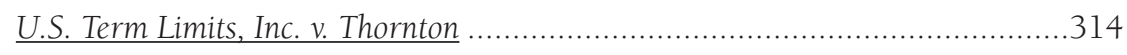

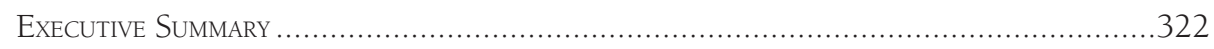

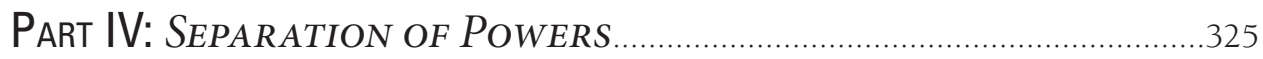




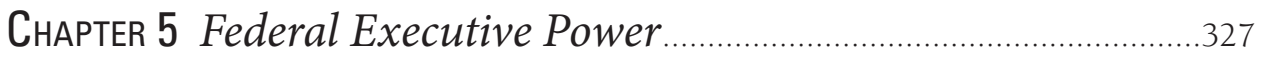

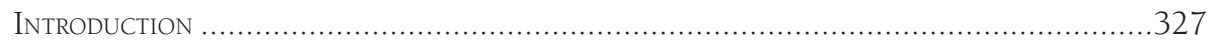

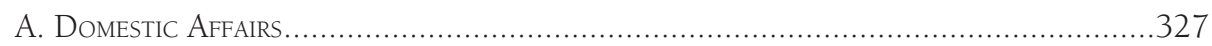

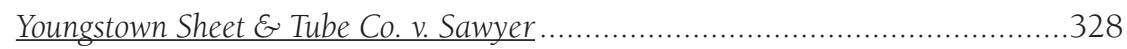

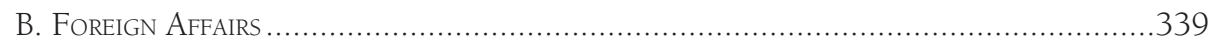

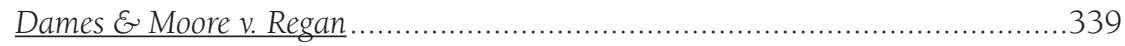

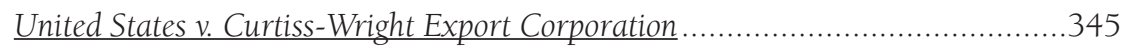

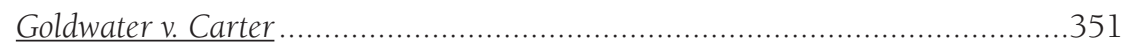

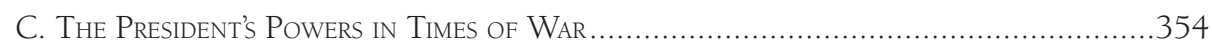

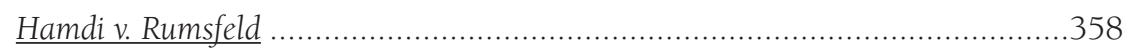

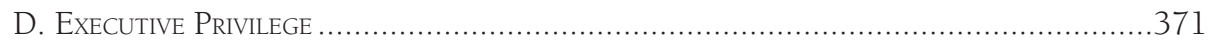

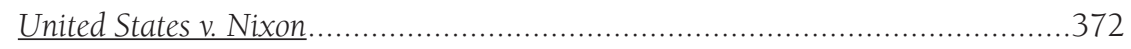

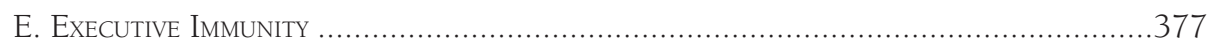

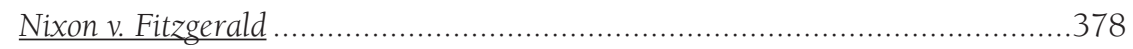

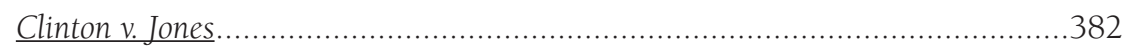

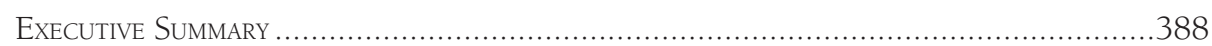

\section{Point-Counterpoint}

Has the Court struck the right balance between the President's power and Congress's

power in the "war on terror"? ................................................................... 391

\section{Chapter 6 The Separation of Powers: The Legislative Process....... 395}

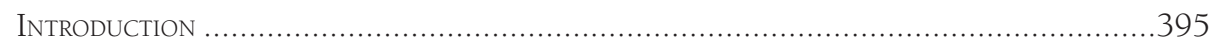

A. Delegation of Legislative Power .............................................................................395

Perspective and Analysis: Richard Pierce .......................................................396

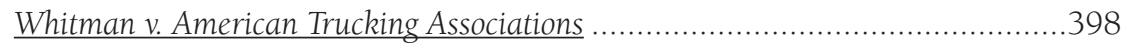

B. Excluding the President from the Legislative Process ............................................403

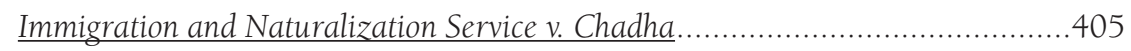

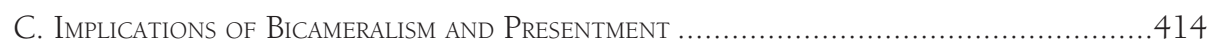

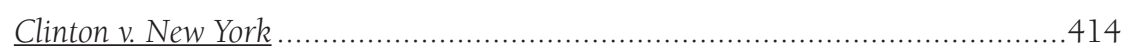

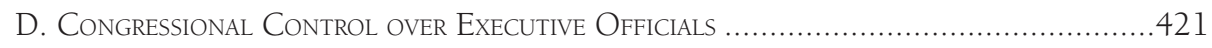

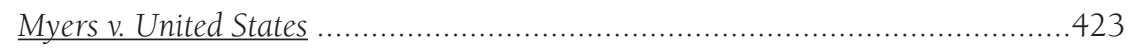

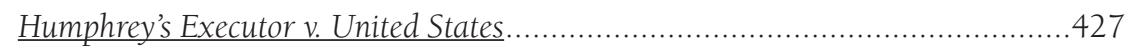

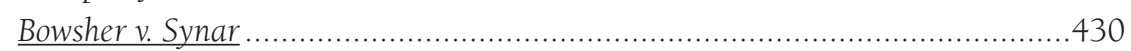

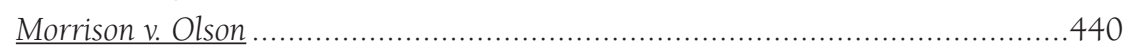

Free Enterprise Fund v. Public Company Accounting Oversight Board ................451

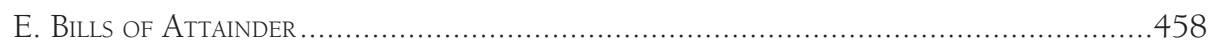

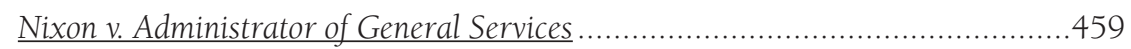

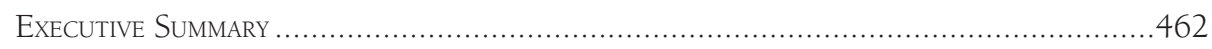


Part V: The Constitution and Individual Rights. 465

CHAPTER 7 Introduction and Background ........................................467

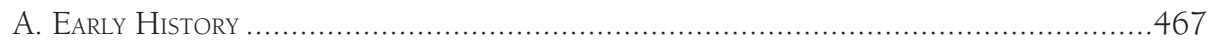

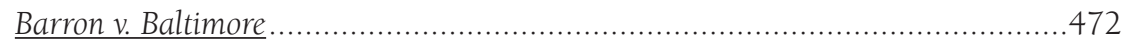

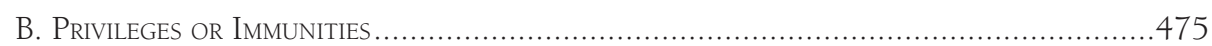

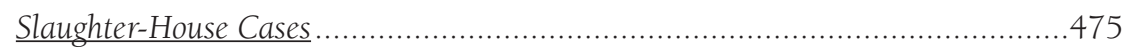

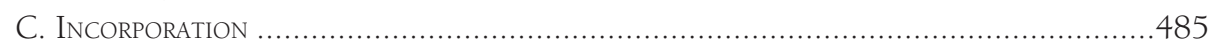

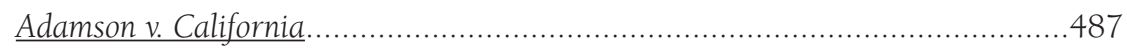

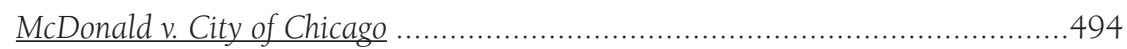

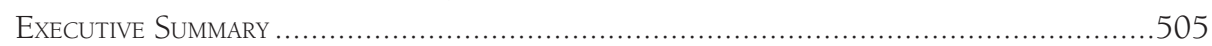

\section{Point-Counterpoint}

Is it too late to question the incorporation doctrine? ................................................507

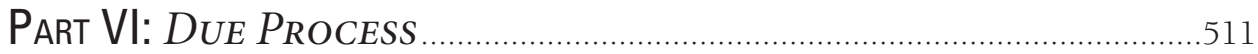

Chapter 8 Substantive Due Process …………………………….........513

A. Substantive Due Process and Economic Liberty ...............................................514

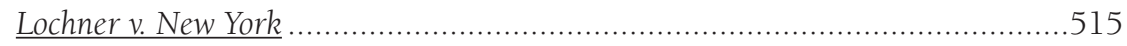

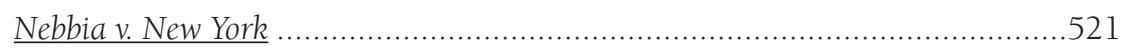

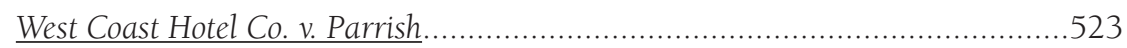

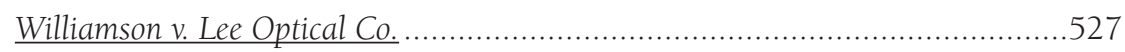

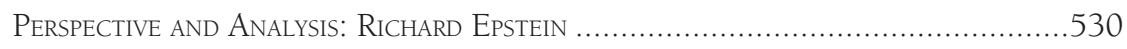

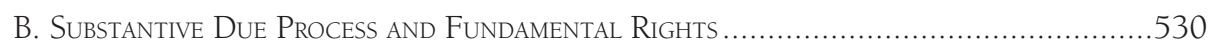

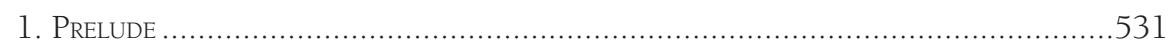

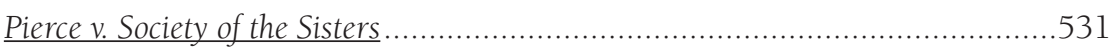

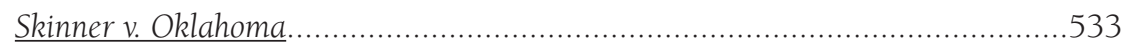

2. Contraception ANd Abortion …………………

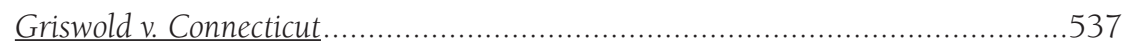

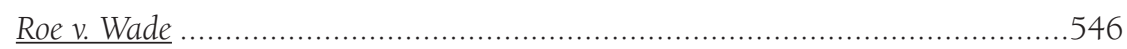

Planned Parenthood of Southeastern Penn. v. Casey .........................................553

Gonzales v. Carhart ...............................................................................56

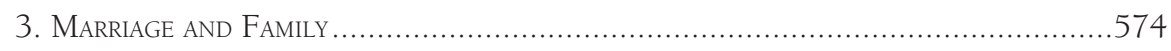

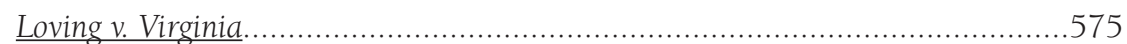

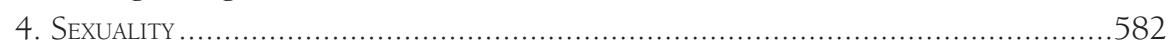

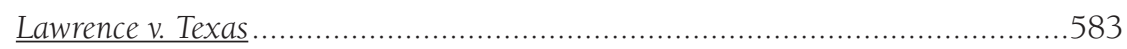


Perspective and Analysis: Robert Delahunty $\&$ John Yoo ..............................591

Perspective And AnAlysis: Vicki JacksOn ....................................................5 592

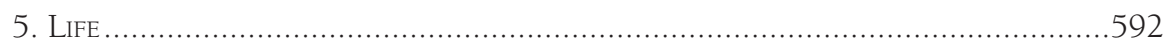

DeShaney v. Winnebago County Dept. of Social Services ....................................592

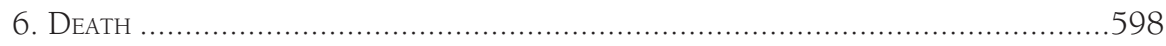

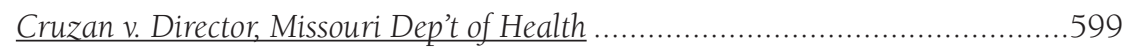

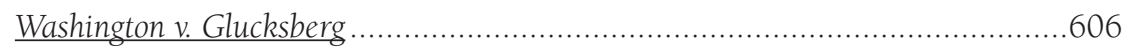

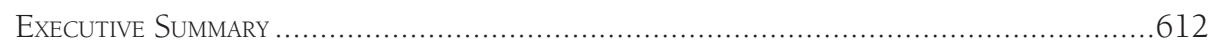

\section{Point-Counterpoint}

Does the Constitution protect unenumerated rights? ....................................615

CHAPTER 9 Procedural Due Process ..................................................

Cleveland Board of Education v. Loudermill .........................................620

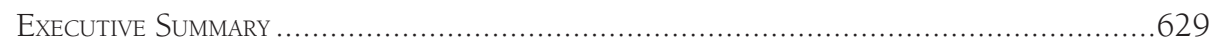

PART VII: EQUAL PRoteCtION ............................................................

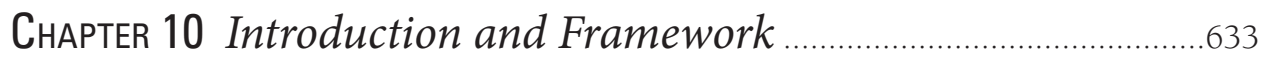

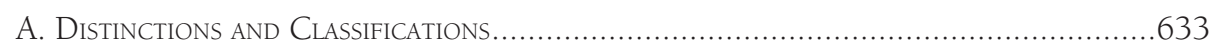

Perspective and Analysis: John Hart Ely ....................................................6 635

B. To Whom Does the Obligation of Equal Protection Apply? ...............................636

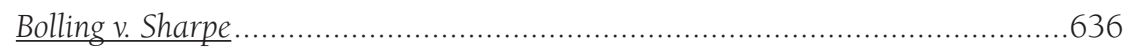

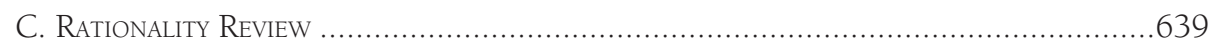

Railway Express Agency v. People of State of New York ..................................639

New York City Transit Authority v. Beazer ...............................................6. 643

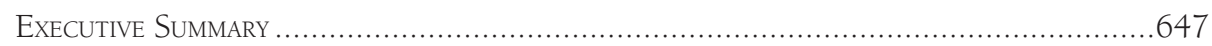

\section{Point-Counterpoint}

Was Bolling v. Sharpe, 347 U.S. 497 (1954), correctly decided? ..............................649

ChAPTER 11 Status-Based Classifications .............................................653

A. Classifications Based on Race and National Origin ...........................................654

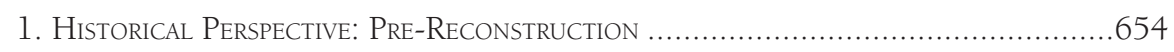

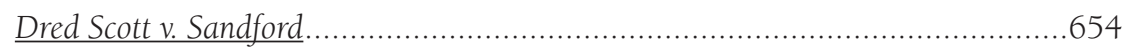

2. Facial Discrimination Against Minorities ...............................................658 


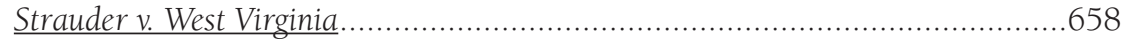

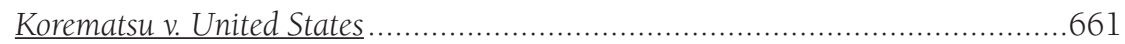

3. Discriminatory Application of Facially Neutral LaWs .....................................68

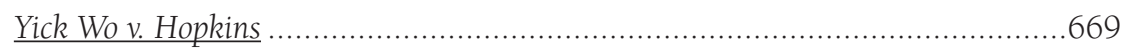

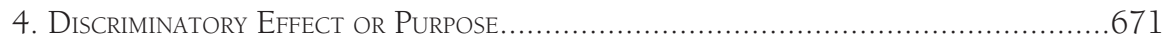

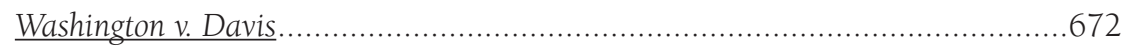

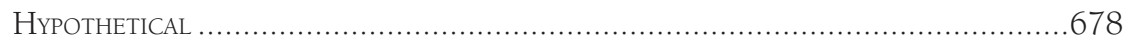

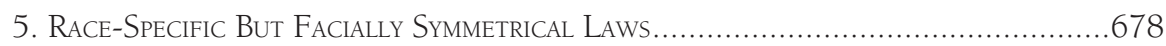

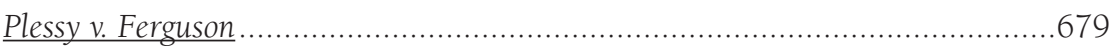

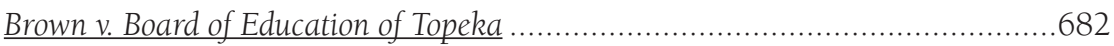

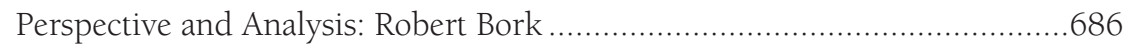

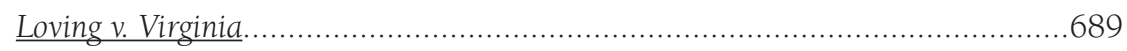

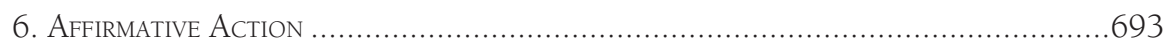

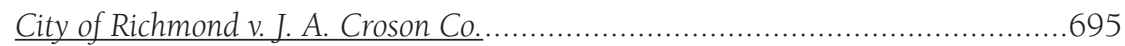

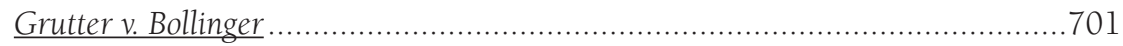

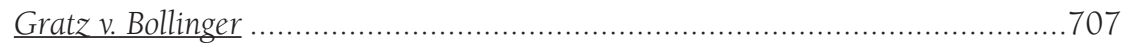

Parents Involved in Community Schools v. Seattle School Dist. No. 1 ...................712

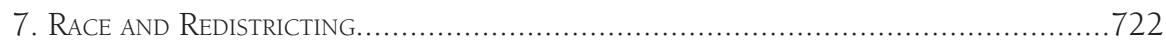

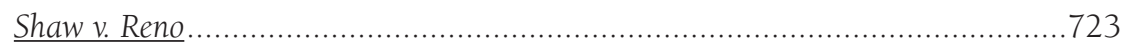

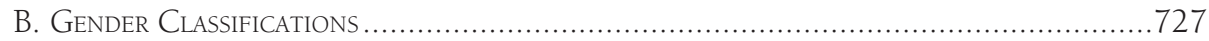

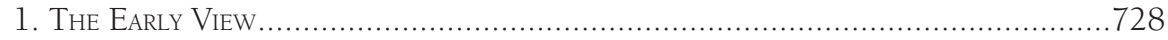

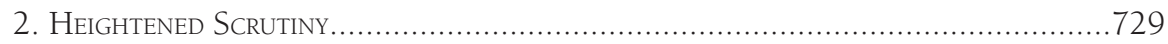

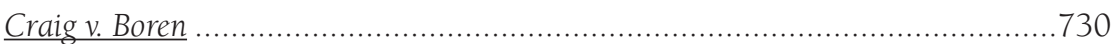

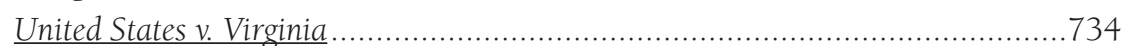

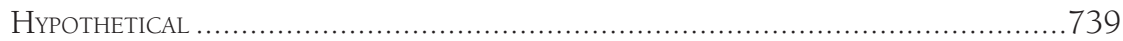

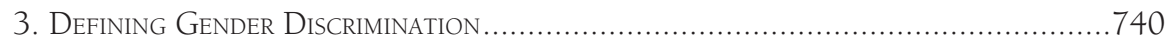

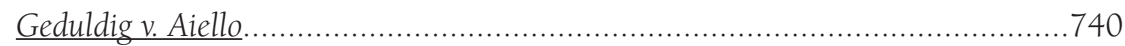

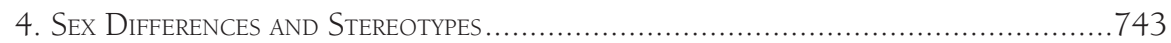

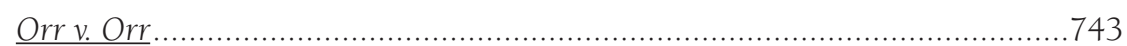

Michael M. v. Superior Court of Sonoma County .............................................746

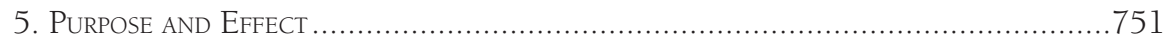

Personnel Administrator of Massachusetts v. Feeney ........................................751

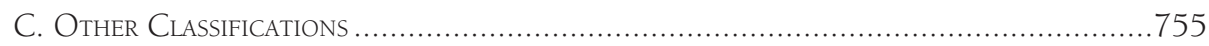

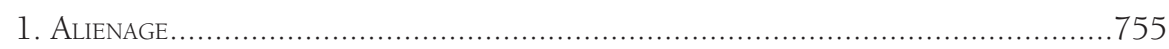

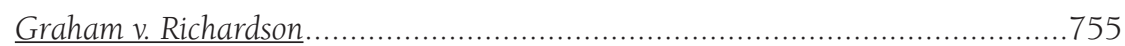

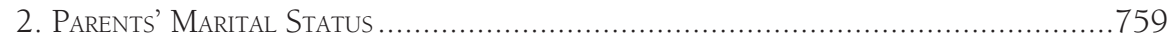

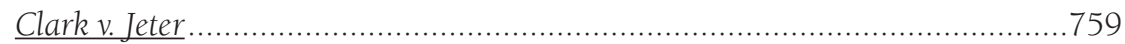

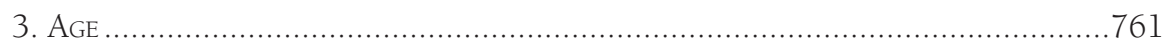

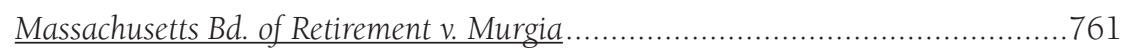

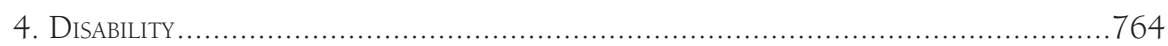




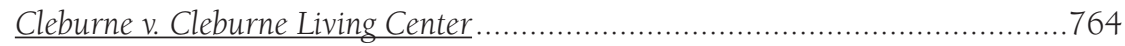

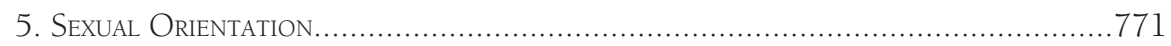

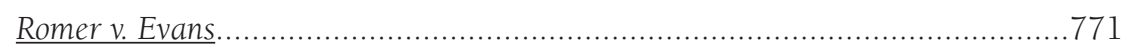

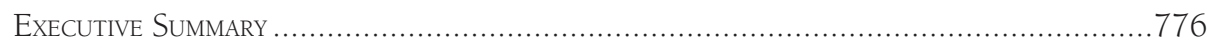

\section{Chapter 12 Equal Protection and Fundamental Rights ...................779}

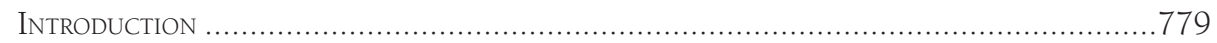

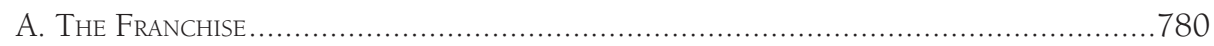

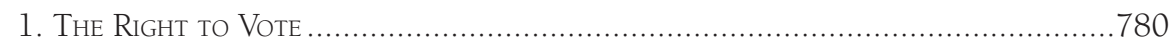

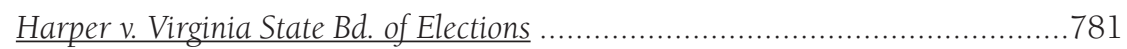

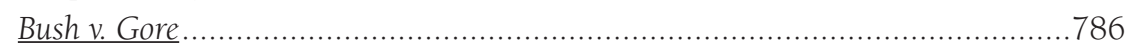

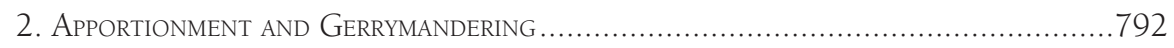

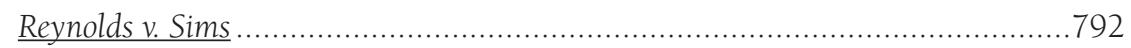

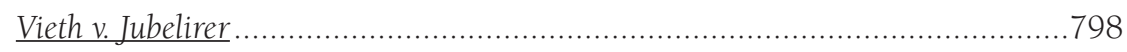

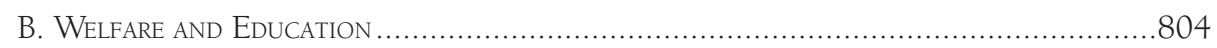

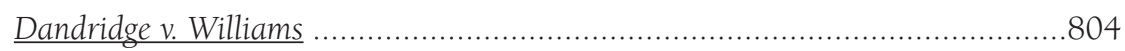

San Antonio Independent School Dist. v. Rodriguez .........................................807

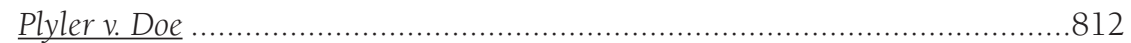

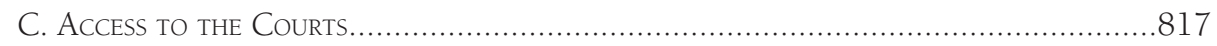

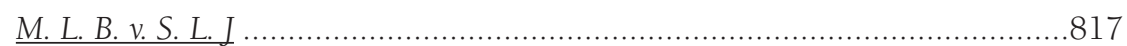

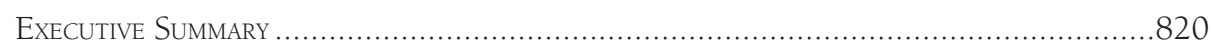

Part VIII: Legislative Protection of Individual Rights ...........821

\section{CHAPTER 13 Congress's Power to Enforce the Reconstruction}

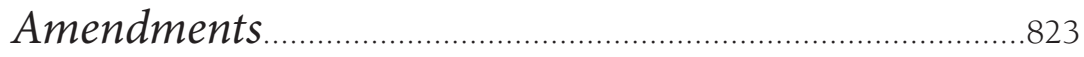

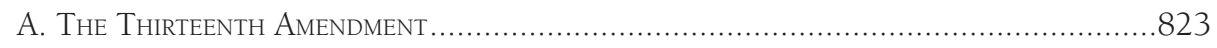

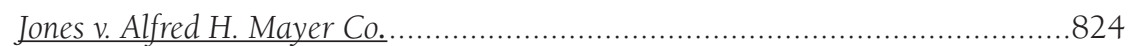

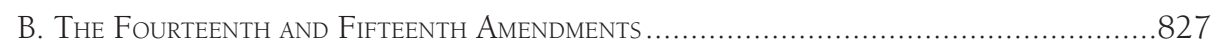

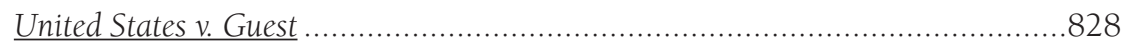

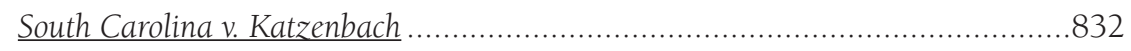

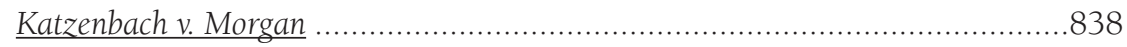

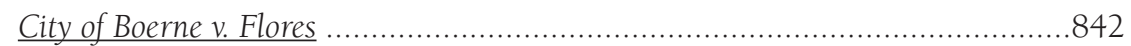

Perspective and Analysis: Robert Post and Reva Siegel ..................................846

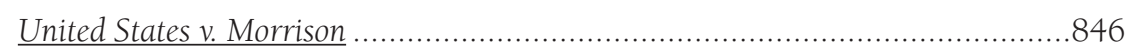

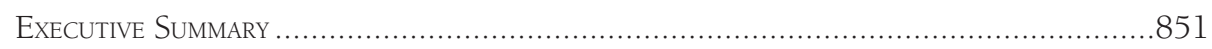




\section{Point-Counterpoint}

Should the Reconstruction Amendments be read in light of the Tenth Amendment? ....853

\section{Part IX: The First Amendment}

\section{ChAPTER 14 Freedom of Speech}

INTRODUCTION 859

A. General Principles and Rules for Regulating Speech .862

1. Content-Based Restrictions 863

Renton v. Playtime Theatres, Inc. 865

Turner Broadcasting System, Inc. v. F.C.C. .869

Republican Party of Minnesota v. White. .873

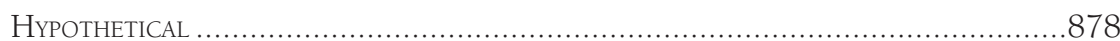

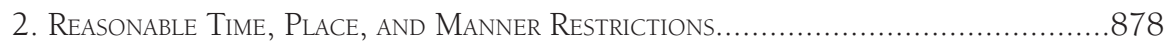

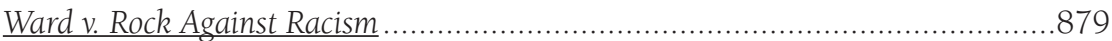

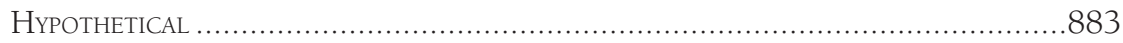

3. Generally Applicable Regulations that Incidentally Affect Expression .............883

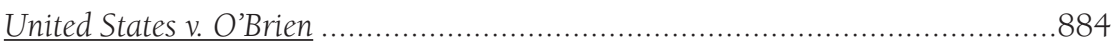

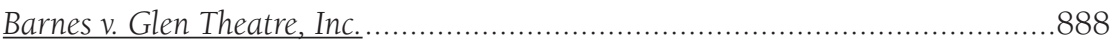

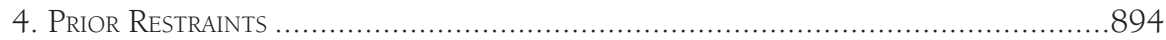

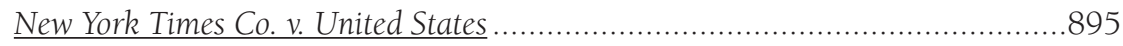

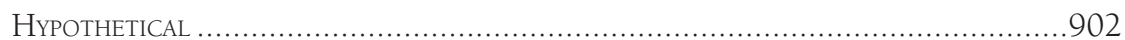

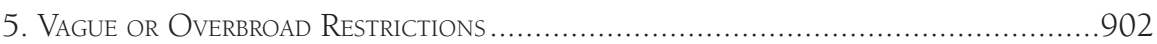

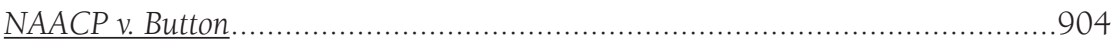

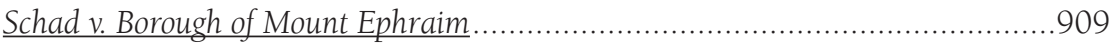

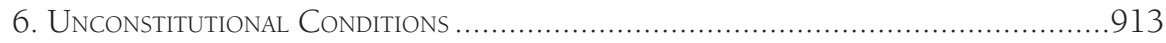

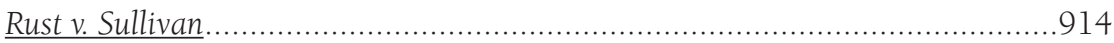

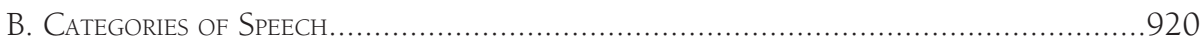

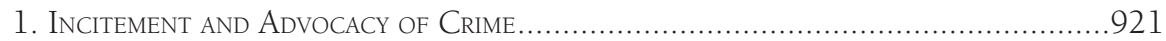

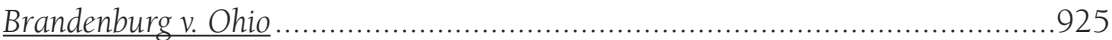

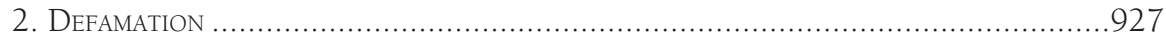

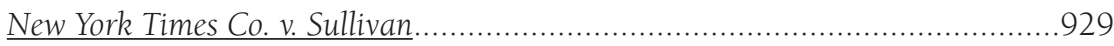

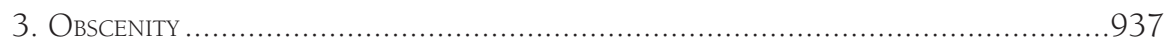

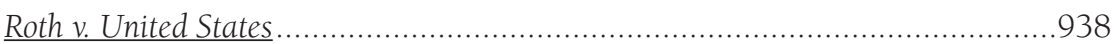

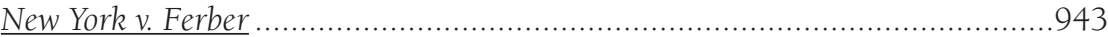

4. Symbolic Conduct …………………………

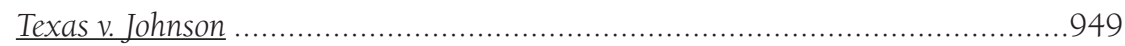

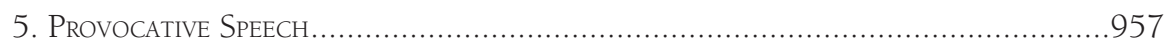

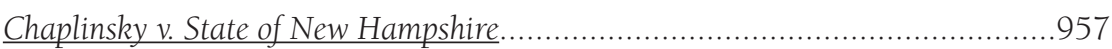




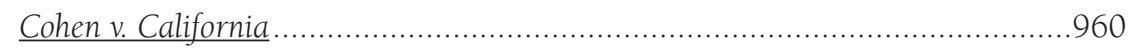

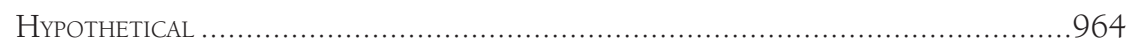

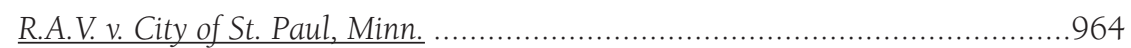

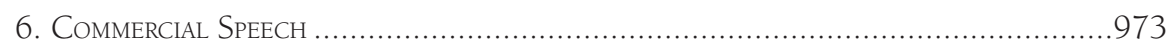

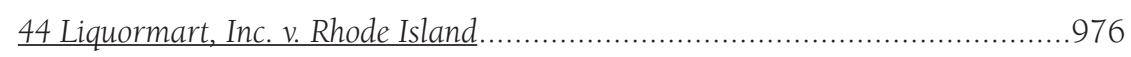

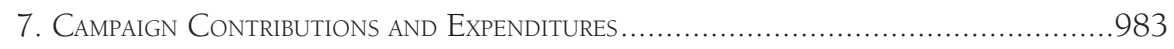

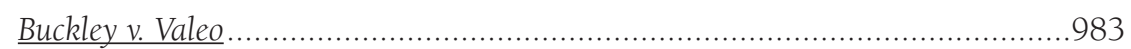

Citizens United v. Federal Election Commission..............................................992

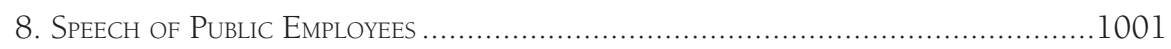

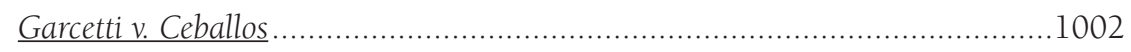

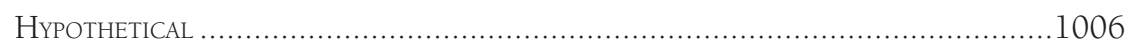

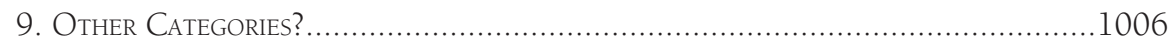

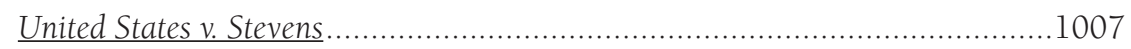

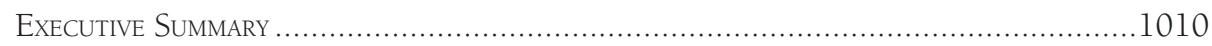

CHAPTER 15 Freedom of the Press

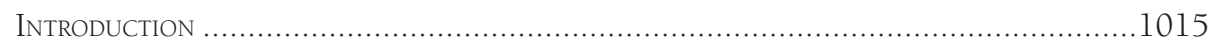

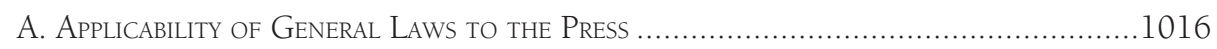

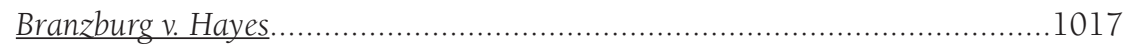

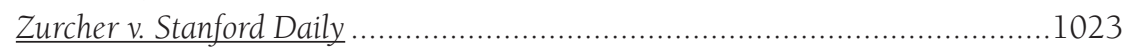

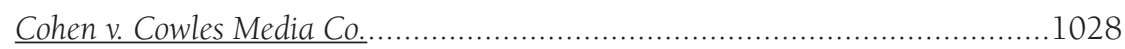

B. Requiring the Press to Provide Media Access to Others ....................................1031

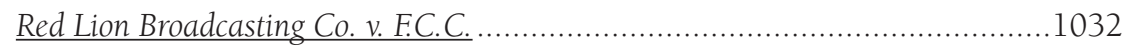

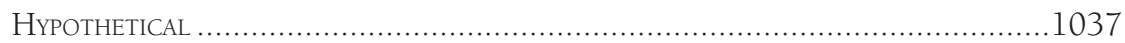

Miami Herald Publishing Co. v. Tornillo ..........................................................1038

C. Press Access to Government Proceedings ..........................................................1042

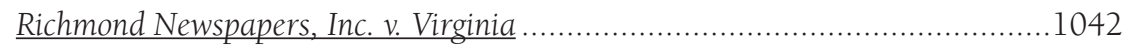

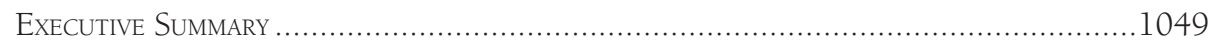

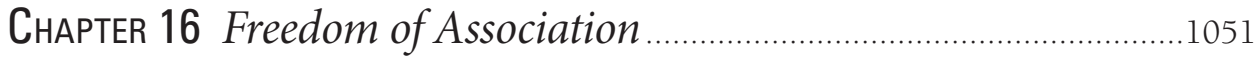

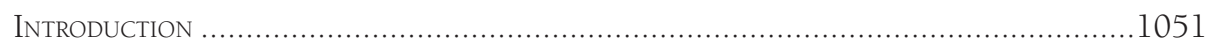

A. Penalizing Individuals for Joining Groups .........................................................1053

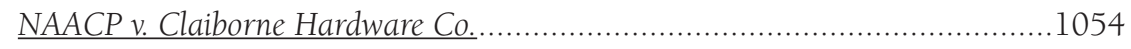

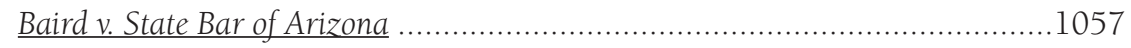

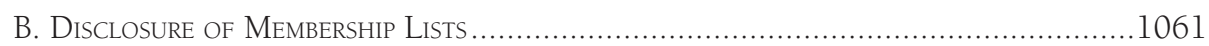

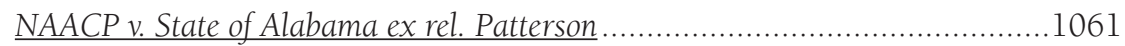

C. Freedom Not to Associate with Others ..........................................................1066

Hurley v. Irish-American Gay, Lesbian and Bisexual Group of Boston ................ 1067

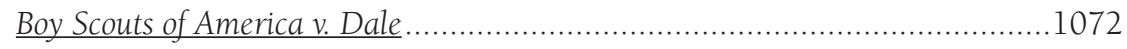




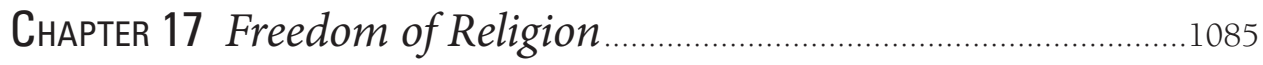

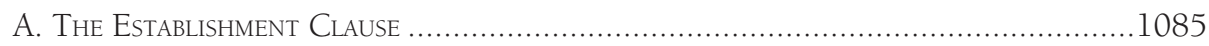

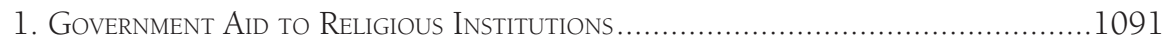

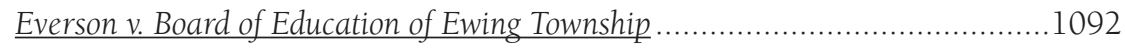

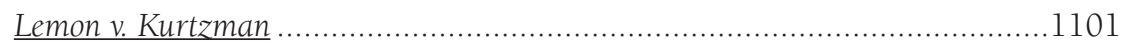

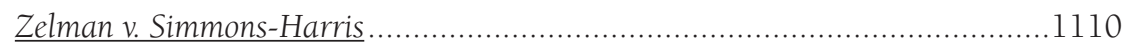

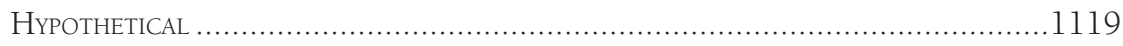

A Note on Standing and Establishment Clause Challenges ............................1119

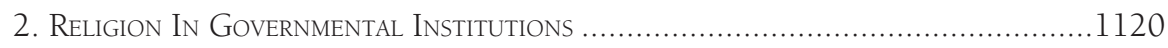

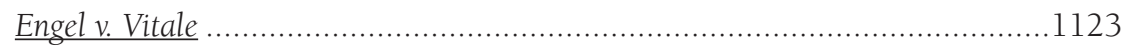

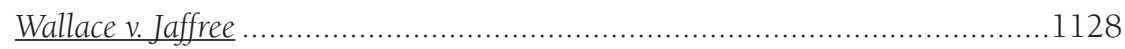

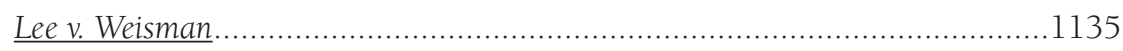

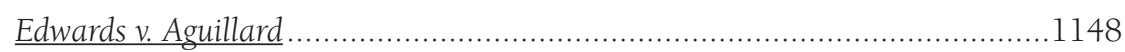

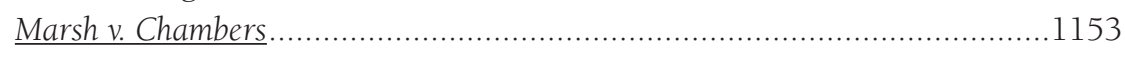

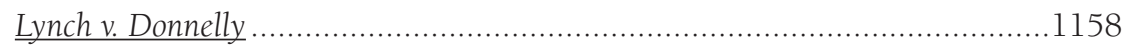

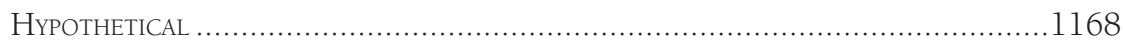

McCreary County, Kentucky v. American Civil Liberties Union of Kentucky.......1169

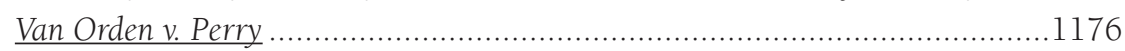

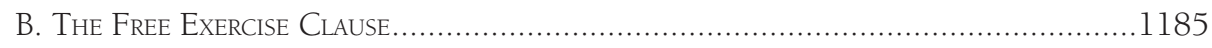

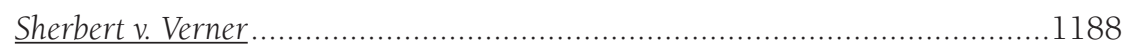

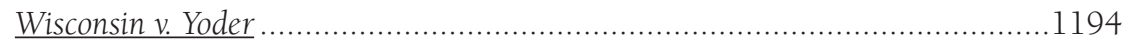

Employment Div. Dept. of Human Resources of Oregon v. Smith ...................... 1200

Church of the Lukumi Babalu Aye, Inc. v. City of Hialeah ................................1211

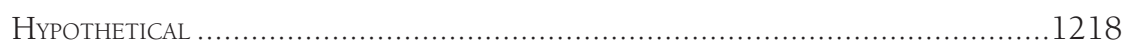

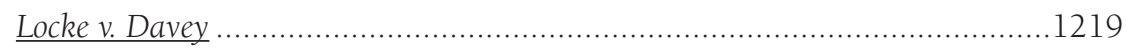

C. Government Involvement in Religious Disputes...............................................1223

Presbyterian Church in U.S. v. Mary Elizabeth Blue Hull Memorial Presbyterian Church ....................................................................1224

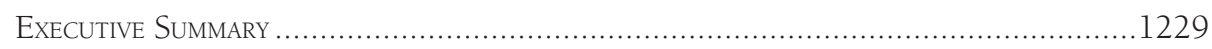

\section{Point-Counterpoint}

Has the Court properly answered the question, "Whom should the First Amendment protect"? 
ChaPTER 18 Protection of Economic Liberty ……………………........1237

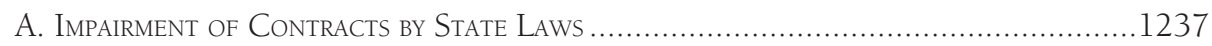

Home Building \& Loan Ass'n v. Blaisdell .....................................................1238

Allied Structural Steel Company v. Spannaus ................................................1241

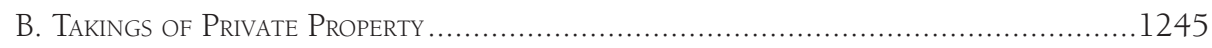

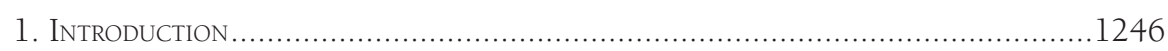

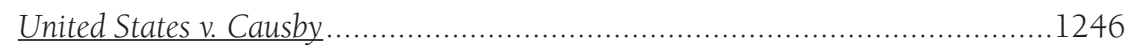

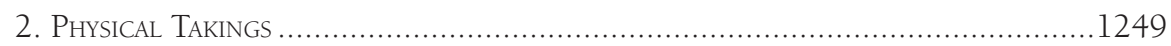

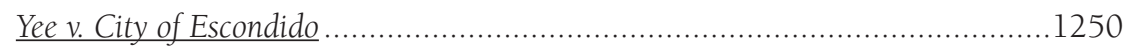

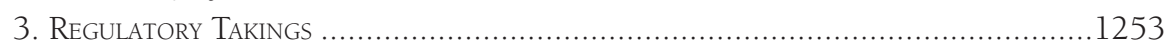

Penn Central Transportation Co. v. City of New York ....................................1253

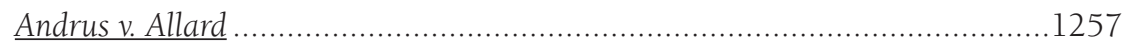

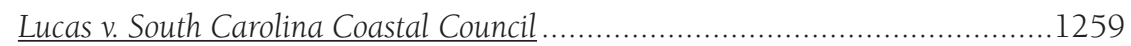

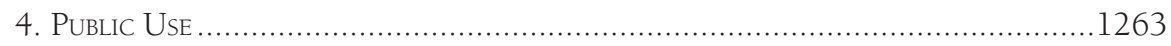

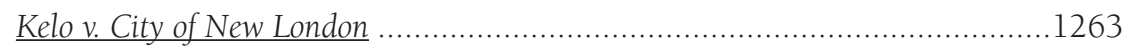

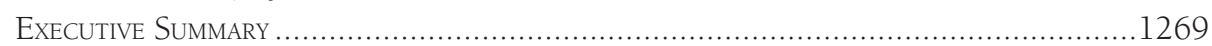

Part XI: Constitutional Limitations on

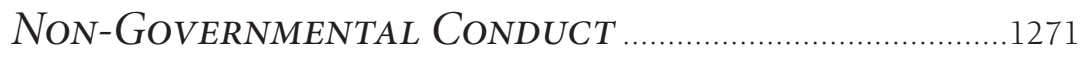

Chapter 19 The State Action Doctrine …....................................1273

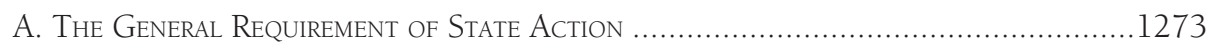

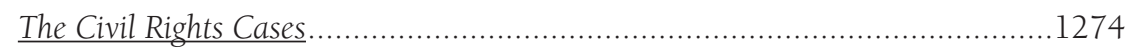

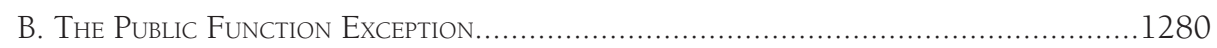

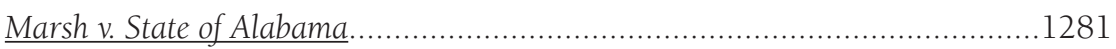

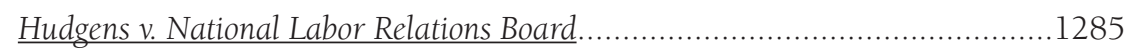

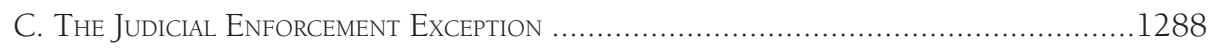

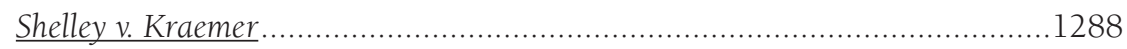

D. The Joint Participation (or Entanglement) Exception.......................................1292

Burton v. Wilmington Parking Authority ....................................................1293

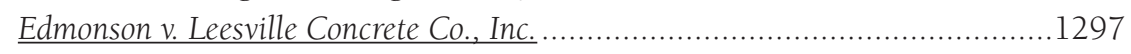

National Collegiate Athletic Association v. Tarkanian ......................................1300

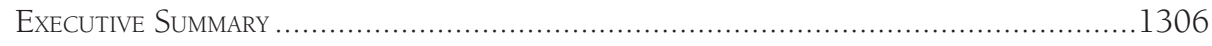

APPENDIX The Constitution of the United States of America..........1307

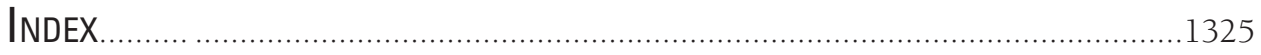




\section{Table of Cases}

The principal cases are in bold type. Cases cited or discussed in the text are in roman type. References are to pages. Cases cited in principal cases and within other quoted materials are not included.

Abbott Laboratories v. Gardner, 387 U.S. 136, 87 S.Ct. 1507, 18 L.Ed.2d 681 (1967), 82

Abrams v. United States, 250 U.S. 616, 40 S.Ct. 17, 63 L.Ed. 1173 (1919), 862

Adair v. United States, 208 U.S. 161, 28 S.Ct. 277, 52 L.Ed. 436 (1908), 520

Adamson v. People of State of California, 332 U.S. 46, 67 S.Ct. 1672, 91 L.Ed. 1903 (1947), 487

Adarand Constructors, Inc. v. Pena, 515 U.S. 200, 115 S.Ct. 2097, 132 L.Ed.2d 158 (1995), 639, 693,700

Adkins v. Children's Hospital of the District of Columbia, 261 U.S. 525, 43 S.Ct. 394, 67 L.Ed. 785 (1923), 520

Aetna Life Ins. Co. of Hartford, Conn. v. Haworth, 300 U.S. 227, 57 S.Ct. 461, 81 L.Ed. 617 (1937), 81

A.L.A. Schechter Poultry Corporation v. United States, 295 U.S. 495, 55 S.Ct. 837, 79 L.Ed. 1570 (1935), 145, 398

Alden v. Maine, 527 U.S. 706, 119 S.Ct. 2240, 144 L.Ed.2d 636 (1999), 229

Alexander v. United States, 509 U.S. 544, 113 S.Ct. 2766, 125 L.Ed.2d 441 (1993), 894

Allegheny, County of v. American Civil Liberties Union Greater Pittsburgh Chapter, 492 U.S. 573, 109 S.Ct. 3086, 106 L.Ed.2d 472 (1989), 1135, 1168

Allen v. Wright, 468 U.S. 737, 104 S.Ct. 3315, 82 L.Ed.2d 556 (1984), 83, 1119

Allied Structural Steel Co. v. Spannaus, 438 U.S. 234, 98 S.Ct. 2716, 57 L.Ed.2d 727 (1978), 1241

American Jewish Congress v. City of Chicago, 827 F.2d 120 (7th Cir.1987), 1168

Andrus v. Allard, 444 U.S. 51, 100 S.Ct. 318, 62 L.Ed.2d 210 (1979), 1257

Arkansas Elec. Co-op. Corp. v. Arkansas Public Service Com'n, 461 U.S. 375, 103 S.Ct. 1905, 76 L.Ed.2d 1 (1983), 275

Arlington Heights, Village of v. Metropolitan Housing Development Corp., 429 U.S. 252, 97 S.Ct. 555, 50 L.Ed.2d 450 (1977), 675

Ashcroft v. Free Speech Coalition, 535 U.S. 234, 122 S.Ct. 1389, 152 L.Ed.2d 403 (2002), 902, 947

Avery v. Midland County, Tex., 390 U.S. 474, 88 S.Ct. 1114, 20 L.Ed.2d 45 (1968), 796

Baird v. State Bar of Ariz., 401 U.S. 1, 91 S.Ct. 702, 27 L.Ed.2d 639 (1971), 1057 
Baker v. Carr, 369 U.S. 186, 82 S.Ct. 691, 7 L.Ed.2d 663 (1962), 68, 792

Baldwin v. Fish and Game Commission of Montana, 436 U.S. 371, 98 S.Ct. 1852, 56 L.Ed.2d 354 (1978), 309, 310

Ballard, United States v., 322 U.S. 78, 64 S.Ct. 882, 88 L.Ed. 1148 (1944), 1186

Barnes v. Glen Theatre, Inc., 501 U.S. 560, 111 S.Ct. 2456, 115 L.Ed.2d 504 (1991), 888, 913

Barron v. City of Baltimore, 32 U.S. 243, 8 L.Ed. 672 (1833), 472

Batson v. Kentucky, 476 U.S. 79, 106 S.Ct. 1712, 90 L.Ed.2d 69 (1986), 660

Beauharnais v. People of State of Ill., 343 U.S. 250, 72 S.Ct. 725, 96 L.Ed. 919 (1952), 928, 1011

Benton v. Maryland, 395 U.S. 784, 89 S.Ct. 2056, 23 L.Ed.2d 707 (1969), 492

Best \& Co. v. Maxwell, 311 U.S. 454, 61 S.Ct. 334, 85 L.Ed. 275 (1940), 264

Blatchford v. Native Village of Noatak and Circle Village, 501 U.S. 775, 111 S.Ct. 2578, 115 L.Ed.2d 686 (1991), 237

Board of County Com'rs, Wabaunsee County, Kan. v. Umbehr, 518 U.S. 668, 116 S.Ct. 2342, 135 L.Ed.2d 843 (1996), 913, 1001

Board of Ed. of Central School Dist. No. 1 v. Allen, 392 U.S. 236, 88 S.Ct. 1923, 20 L.Ed.2d 1060 (1968), 1091, 1105

Board of Educ. of Kiryas Joel Village School Dist. v. Grumet, 512 U.S. 687, 114 S.Ct. 2481, 129 L.Ed.2d 546 (1994), 1219

Board of Regents of State Colleges v. Roth, 408 U.S. 564, 92 S.Ct. 2701, 33 L.Ed.2d 548 (1972), 626 Board of Trustees of University of Alabama v. Garrett, 531 U.S. 356, 121 S.Ct. 955, 148 L.Ed.2d 866 (2001), 238, 770

Bob Jones University v. United States, 461 U.S. 574, 103 S.Ct. 2017, 76 L.Ed.2d 157 (1983), 88

Boerne, City of v. Flores, 521 U.S. 507, 117 S.Ct. 2157, 138 L.Ed.2d 624 (1997), 842, 1211

Bollard v. California Province of the Society of Jesus, 196 F.3d 940 (9th Cir.1999), 1228

Bolling v. Sharpe, 347 U.S. 497, 74 S.Ct. 693, 98 L.Ed. 884 (1954), 631, 636, 649

Boos v. Barry, 485 U.S. 312, 108 S.Ct. 1157, 99 L.Ed.2d 333 (1988), 863, 868

Boumediene v. Bush, 553 U.S. 723, 128 S.Ct. 2229, 171 L.Ed.2d 41 (2008), 101, 371

Bowers v. DeVito, 686 F.2d 616 (7th Cir.1982), 596

Bowers v. Hardwick, 478 U.S. 186, 106 S.Ct. 2841, 92 L.Ed.2d 140 (1986), 582

Bowsher v. Synar, 478 U.S. 714, 106 S.Ct. 3181, 92 L.Ed.2d 583 (1986), 430

Boy Scouts of America v. Dale, 530 U.S. 640, 120 S.Ct. 2446, 147 L.Ed.2d 554 (2000), 1072, 1083

Bradwell v. People of State of Illinois, 83 U.S. 130, 21 L.Ed. 442 (1872), 20, 728

Brandenburg v. Ohio, 395 U.S. 444, 89 S.Ct. 1827, 23 L.Ed.2d 430 (1969), 925, 1011, 1060

Branzburg v. Hayes, 408 U.S. 665, 92 S.Ct. 2646, 33 L.Ed.2d 626 (1972), 1016, 1017, 1049

Braunfeld v. Brown, 366 U.S. 599, 81 S.Ct. 1144, 6 L.Ed.2d 563 (1961), 1186, 1187

Breard v. City of Alexandria, La., 341 U.S. 622, 71 S.Ct. 920, 95 L.Ed. 1233 (1951), 973

Brown v. Board of Ed. of Topeka, Shawnee County, Kan., 347 U.S. 483, 74 S.Ct. 686, 98 L.Ed. 873 (1954), 20, 682, 1273

Brown v. Board of Educ. of Topeka, Kan., 349 U.S. 294, 75 S.Ct. 753, 99 L.Ed. 1083 (1955), 688

Buck v. Bell, 274 U.S. 200, 47 S.Ct. 584, 71 L.Ed. 1000 (1927), 533, 633

Buckley v. Valeo, 424 U.S. 1, 96 S.Ct. 612, 46 L.Ed.2d 659 (1976), 14, 442, 638, 816, 983, 1013, 1065,1271 
Burton v. Wilmington Parking Authority, 365 U.S. 715, 81 S.Ct. 856, 6 L.Ed.2d 45 (1961), 1293, 1306

Bush v. Gore, 531 U.S. 98, 121 S.Ct. 525, 148 L.Ed.2d 388 (2000), 786

Bush v. Vera, 517 U.S. 952, 116 S.Ct. 1941, 135 L.Ed.2d 248 (1996), 726

Butler, United States v., 297 U.S. 1, 56 S.Ct. 312, 80 L.Ed. 477 (1936), 193

Calder v. Bull, 3 U.S. 386, 3 Dall. 386, 1 L.Ed. 648 (1798), 469

Califano v. Webster, 430 U.S. 313, 97 S.Ct. 1192, 51 L.Ed.2d 360 (1977), 745

California, United States v., 436 U.S. 32, 98 S.Ct. 1662, 56 L.Ed.2d 94 (1978), 328

Camps Newfound/Owatonna, Inc. v. Town of Harrison, Me., 520 U.S. 564, 117 S.Ct. 1590, 137 L.Ed.2d 852 (1997), 287

Cantwell v. State of Connecticut, 310 U.S. 296, 60 S.Ct. 900, 84 L.Ed. 1213 (1940), 492, 1086, $1089,1186,1229$

Capital Cities Cable, Inc. v. Crisp, 467 U.S. 691, 104 S.Ct. 2694, 81 L.Ed.2d 580 (1984), 247

Carmack, United States v., 329 U.S. 230, 67 S.Ct. 252, 91 L.Ed. 209 (1946), 1245

Carolene Products Co., United States v., 304 U.S. 144, 58 S.Ct. 778, 82 L.Ed. 1234 (1938), 526, 634,811

Carter v. Carter Coal Co., 298 U.S. 238, 56 S.Ct. 855, 80 L.Ed. 1160 (1936), 146

Castle Rock, Colo., Town of v. Gonzales, 545 U.S. 748, 125 S.Ct. 2796, 162 L.Ed.2d 658 (2005), 598,627

Causby, United States v., 328 U.S. 256, 66 S.Ct. 1062, 90 L.Ed. 1206 (1946), 1246, 1259

Central Hudson Gas \& Elec. Corp. v. Public Service Commission of New York, 447 U.S. 557, 100 S.Ct. 2343, 65 L.Ed.2d 341 (1980), 974, 1012

Champion v. Ames, 188 U.S. 321, 23 S.Ct. 321, 47 L.Ed. 492 (1903), 139

Chaplinsky v. State of New Hampshire, 315 U.S. 568, 62 S.Ct. 766, 86 L.Ed. 1031 (1942), 920, 957, 1012

Charles C. Steward Mach. Co. v. Davis, 301 U.S. 548, 57 S.Ct. 883, 81 L.Ed. 1279 (1937), 196

Chicago, B. \& Q.R. Co. v. City of Chicago, 166 U.S. 226, 17 S.Ct. 581, 41 L.Ed. 979 (1897), 486, 493,1245

Chicago, City of v. Morales, 527 U.S. 41, 119 S.Ct. 1849, 144 L.Ed.2d 67 (1999), 1053

Child Labor Tax Case, 259 U.S. 20, 42 S.Ct. 449, 66 L.Ed. 817 (1922), 187

Chisholm v. Georgia, 2 U.S. 419, 2 Dall. 419, 1 L.Ed. 440 (1793), 65, 229

Church of American Knights of Ku Klux Klan v. City of Gary, Indiana, 334 F.3d 676 (7th Cir.2003), 959

Church of the Lukumi Babalu Aye, Inc. v. City of Hialeah, 508 U.S. 520, 113 S.Ct. 2217, 124 L.Ed.2d 472 (1993), 844, 1187, 1211, 1230

Citizens Against Rent Control/Coalition for Fair Housing v. City of Berkeley, Cal., 454 U.S. 290, 102 S.Ct. 434, 70 L.Ed.2d 492 (1981), 1052

Citizens United v. Federal Election Commission, __ U.S. _ , 130 S.Ct. 876, 175 L.Ed.2d 753 (2010), 992, 1013, 1065

City of (see name of city)

Civil Rights Cases, 109 U.S. 3, 3 S.Ct. 18, 27 L.Ed. 835 (1883), 828, 1271, 1274, 1306 
Clark v. Community for Creative NonBViolence, 468 U.S. 288, 104 S.Ct. 3065, 82 L.Ed.2d 221 (1984), 888

Clark v. Jeter, 486 U.S. 456, 108 S.Ct. 1910, 100 L.Ed.2d 465 (1988), 759

Cleburne, Tex., City of v. Cleburne Living Center, 473 U.S. 432, 105 S.Ct. 3249, 87 L.Ed.2d 313 (1985), 528, 764

Cleveland Bd. of Educ. v. Loudermill, 470 U.S. 532, 105 S.Ct. 1487, 84 L.Ed.2d 494 (1985), 620

Clinton v. City of New York, 524 U.S. 417, 118 S.Ct. 2091, 141 L.Ed.2d 393 (1998), 414

Clinton v. Jones, 520 U.S. 681, 117 S.Ct. 1636, 137 L.Ed.2d 945 (1997), 382

Cohen v. California, 403 U.S. 15, 91 S.Ct. 1780, 29 L.Ed.2d 284 (1971), 960, 1012

Cohen v. Cowles Media Co., 501 U.S. 663, 111 S.Ct. 2513, 115 L.Ed.2d 586 (1991), 1016, 1028, 1049

Cohens v. State of Virginia, 19 U.S. 264, 5 L.Ed. 257 (1821), 54, 59

Collins v. City of Harker Heights, Tex., 503 U.S. 115, 112 S.Ct. 1061, 117 L.Ed.2d 261 (1992), 1042

Colorado Republican Federal Campaign Committee v. Federal Election Com'n, 518 U.S. 604, 116 S.Ct. 2309, 135 L.Ed.2d 795 (1996), 992

Comstock, United States v., __ U.S. __, 130 S.Ct. 1949, 176 L.Ed.2d 878 (2010), 122

Connick v. Myers, 461 U.S. 138, 103 S.Ct. 1684, 75 L.Ed.2d 708 (1983), 1001, 1013

Consolidated Edison Co. of New York, Inc. v. Public Service Commission of New York, 447 U.S. 530, 100 S.Ct. 2326, 65 L.Ed.2d 319 (1980), 864

Cooley v. Board of Wardens of Port of Philadelphia, to Use of Soc for Relief of Distressed Pilots, Their Widows and Children, 53 U.S. 299, 12 How. 299, 13 L.Ed. 996 (1851), 258, 259

Cooper v. Aaron, 358 U.S. 1, 78 S.Ct. 1401, 3 L.Ed.2d 5, 3 L.Ed.2d 19 (1958), 61

Coppage v. State of Kansas, 236 U.S. 1, 35 S.Ct. 240, 59 L.Ed. 441 (1915), 520

County of (see name of county)

Craig v. Boren, 429 U.S. 190, 97 S.Ct. 451, 50 L.Ed.2d 397 (1976), 730

Crane v. New York, 239 U.S. 195, 36 S.Ct. 85, 60 L.Ed. 218 (1915), 756

Cruzan by Cruzan v. Director, Missouri Dept. of Health, 497 U.S. 261, 110 S.Ct. 2841, 111 L.Ed.2d 224 (1990), 599

Cuellar, United States v., 478 F.3d 282 (5th Cir.2007), 397

CurtissBWright Export Corporation, United States v., 299 U.S. 304, 57 S.Ct. 216, 81 L.Ed. 255 (1936), 345

Dames \& Moore v. Regan, 453 U.S. 654, 101 S.Ct. 2972, 69 L.Ed.2d 918 (1981), 339

Dandridge v. Williams, 397 U.S. 471, 90 S.Ct. 1153, 25 L.Ed.2d 491 (1970), 804

Darby, United States v., 312 U.S. 100, 312 U.S. 657, 61 S.Ct. 451, 85 L.Ed. 609 (1941), 152

Davis v. Bandemer, 478 U.S. 109, 106 S.Ct. 2797, 92 L.Ed.2d 85 (1986), 797

Davis, Helvering v., 301 U.S. 619, 301 U.S. 672, 57 S.Ct. 904, 81 L.Ed. 1307 (1937), 196

Dean Milk Co. v. City of Madison, Wis., 340 U.S. 349, 71 S.Ct. 295, 95 L.Ed. 329 (1951), 265

Dennis v. Sparks, 449 U.S. 24, 101 S.Ct. 183, 66 L.Ed.2d 185 (1980), 1305

Dennis v. United States, 341 U.S. 494, 71 S.Ct. 857, 95 L.Ed. 1137 (1951), 921

Denver Area Educational Telecommunications Consortium, Inc. v. F.C.C., 518 U.S. 727, 116 S.Ct. 2374, 135 L.Ed.2d 888 (1996), 971 
DeShaney v. Winnebago County Dept. of Social Services, 489 U.S. 189, 109 S.Ct. 998, 103 L.Ed.2d 249 (1989), 592

Dickerson v. United States, 530 U.S. 428, 120 S.Ct. 2326, 147 L.Ed.2d 405 (2000), 64

District of Columbia v. Heller, 554 U.S. 570, 128 S.Ct. 2783, 171 L.Ed.2d 637 (2008), 23, 493, 646

Doe v. Bolton, 410 U.S. 179, 93 S.Ct. 739, 35 L.Ed.2d 201 (1973), 309, 551

Dorsey, United States v., 418 F.3d 1038 (9th Cir.2005), 172

Douglas v. People of State of Cal., 372 U.S. 353, 83 S.Ct. 814, 9 L.Ed.2d 811 (1963), 807

Dred Scott v. Sandford, 60 U.S. 393, 19 How. 393, 15 L.Ed. 691 (1856), 64, 65, 514, 654

Dun \& Bradstreet, Inc. v. Greenmoss Builders, Inc., 472 U.S. 749, 105 S.Ct. 2939, 86 L.Ed.2d 593 (1985), 936

Dyer v. Blair, 390 F.Supp. 1291 (N.D.Ill.1975), 353

Easley v. Cromartie, 532 U.S. 234, 121 S.Ct. 1452, 149 L.Ed.2d 430 (2001), 726

E. C. Knight Co., United States v., 156 U.S. 1, 15 S.Ct. 249, 39 L.Ed. 325 (1895), 134

Edmonson v. Leesville Concrete Co., Inc., 500 U.S. 614, 111 S.Ct. 2077, 114 L.Ed.2d 660 (1991), 661, 1297

Edwards v. Aguillard, 482 U.S. 578, 107 S.Ct. 2573, 96 L.Ed.2d 510 (1987), 1148, 1156

Eichman, United States v., 496 U.S. 310, 110 S.Ct. 2404, 110 L.Ed.2d 287 (1990), 956

Eisenstadt v. Baird, 405 U.S. 438, 92 S.Ct. 1029, 31 L.Ed.2d 349 (1972), 546

Elk Grove Unified School Dist. v. Newdow, 542 U.S. 1, 124 S.Ct. 2301, 159 L.Ed.2d 98 (2004), 1089,1168

Employment Div., Dept. of Human Resources of Oregon v. Smith, 494 U.S. 872, 110 S.Ct. 1595, 108 L.Ed.2d 876 (1990), 894, 1186, 1200, 1230

Engel v. Vitale, 370 U.S. 421, 82 S.Ct. 1261, 8 L.Ed.2d 601 (1962), 1085, 1123, 1230

Epperson v. State of Ark., 393 U.S. 97, 89 S.Ct. 266, 21 L.Ed.2d 228 (1968), 1147, 1149

Everson v. Board of Ed. of Ewing Tp., 330 U.S. 1, 67 S.Ct. 504, 91 L.Ed. 711 (1947), 492, 1086, 1088, 1091, 1092, 1219, 1229

Ex parte (see name of party)

Federal Election Com'n v. Akins, 524 U.S. 11, 118 S.Ct. 1777, 141 L.Ed.2d 10 (1998), 96 Felker v. Turpin, 518 U.S. 651, 116 S.Ct. 2333, 135 L.Ed.2d 827 (1996), 101

Ferguson v. Skrupa, 372 U.S. 726, 83 S.Ct. 1028, 10 L.Ed.2d 93 (1963), 529

Flast v. Cohen, 392 U.S. 83, 88 S.Ct. 1942, 20 L.Ed.2d 947 (1968), 1119

Flemming v. Nestor, 363 U.S. 603, 80 S.Ct. 1367, 4 L.Ed.2d 1435 (1960), 643

Fletcher v. Peck, 10 U.S. 87, 3 L.Ed. 162 (1810), 470

Florida Bar v. Went For It, Inc., 515 U.S. 618, 115 S.Ct. 2371, 132 L.Ed.2d 541 (1995), 909

Florida Lime \& Avocado Growers, Inc. v. Paul, 373 U.S. 132, 83 S.Ct. 1210, 10 L.Ed.2d 248 (1963), 246

Foley v. Connelie, 435 U.S. 291, 98 S.Ct. 1067, 55 L.Ed.2d 287 (1978), 758

44 Liquormart, Inc. v. Rhode Island, 517 U.S. 484, 116 S.Ct. 1495, 134 L.Ed.2d 711 (1996), 976

Free Enterprise Fund v. Public Co. Accounting Oversight Bd., __ U.S. __, 130 S.Ct. 3138, 177

L.Ed.2d 706 (2010), 451 
Frontiero v. Richardson, 411 U.S. 677, 93 S.Ct. 1764, 36 L.Ed.2d 583 (1973), 729

Frothingham v. Mellon, 262 U.S. 447, 43 S.Ct. 597, 67 L.Ed. 1078 (1923), 1119

Fullilove v. Klutznick, 448 U.S. 448, 100 S.Ct. 2758, 65 L.Ed.2d 902 (1980), 696

Garcetti v. Ceballos, 547 U.S. 410, 126 S.Ct. 1951, 164 L.Ed.2d 689 (2006), 1002, 1013

Garcia v. San Antonio Metropolitan Transit Authority, 469 U.S. 528, 105 S.Ct. 1005, 83 L.Ed.2d 1016 (1985), 209

Gayle v. Browder, 352 U.S. 903, 77 S.Ct. 145, 1 L.Ed.2d 114 (1956), 685

Geduldig v. Aiello, 417 U.S. 484, 94 S.Ct. 2485, 41 L.Ed.2d 256 (1974), 740

Gentile v. State Bar of Nevada, 501 U.S. 1030, 111 S.Ct. 2720, 115 L.Ed.2d 888 (1991), 903

Georgia v. McCollum, 505 U.S. 42, 112 S.Ct. 2348, 120 L.Ed.2d 33 (1992), 661, 1300

Gertz v. Robert Welch, Inc., 418 U.S. 323, 94 S.Ct. 2997, 41 L.Ed.2d 789 (1974), 928, 935

Gibbons v. Ogden, 22 U.S. 1, 6 L.Ed. 23 (1824), 130, 255

Gibson v. Mississippi, 162 U.S. 565, 16 S.Ct. 904, 40 L.Ed. 1075 (1896), 637

Gideon v. Wainwright, 372 U.S. 335, 83 S.Ct. 792, 9 L.Ed.2d 799 (1963), 493

Gitlow v. People of State of New York, 268 U.S. 652, 45 S.Ct. 625, 69 L.Ed. 1138 (1925), 492, 504, 1015, 1049

Goldberg v. Kelly, 397 U.S. 254, 90 S.Ct. 1011, 25 L.Ed.2d 287 (1970), 626

Goldman v. Weinberger, 475 U.S. 503, 106 S.Ct. 1310, 89 L.Ed.2d 478 (1986), 1193, 1209

Goldwater v. Carter, 444 U.S. 996, 100 S.Ct. 533, 62 L.Ed.2d 428 (1979), 351

Gomillion v. Lightfoot, 364 U.S. 339, 81 S.Ct. 125, 5 L.Ed.2d 110 (1960), 671, 722, 727

Gonzales v. Carhart, 550 U.S. 124, 127 S.Ct. 1610, 167 L.Ed.2d 480 (2007), 568

Gonzales v. O Centro Espirita Beneficente Uniao do Vegetal, 546 U.S. 418, 126 S.Ct. 1211, 163 L.Ed.2d 1017 (2006), 1211

Gonzales v. Oregon, 546 U.S. 243, 126 S.Ct. 904, 163 L.Ed.2d 748 (2006), 610

Gonzales v. Raich, 545 U.S. 1, 125 S.Ct. 2195, 162 L.Ed.2d 1 (2005), 178

Good News Club v. Milford Central School, 533 U.S. 98, 121 S.Ct. 2093, 150 L.Ed.2d 151 (2001), 1147

Goodridge v. Department of Public Health, 440 Mass. 309, 798 N.E.2d 941 (Mass.2003), 590, 776

Goss v. Lopez, 419 U.S. 565, 95 S.Ct. 729, 42 L.Ed.2d 725 (1975), 627

Graham v. Richardson, 403 U.S. 365, 91 S.Ct. 1848, 29 L.Ed.2d 534 (1971), 755

Gratz v. Bollinger, 539 U.S. 244, 123 S.Ct. 2411, 156 L.Ed.2d 257 (2003), 707

Green v. County School Bd. of New Kent County, Va., 391 U.S. 430, 88 S.Ct. 1689, 20 L.Ed.2d 716 (1968), 688

Greenya v. George Washington University, 512 F.2d 556, 167 U.S.App.D.C. 379 (D.C.Cir.1975), 1288

Griffin v. California, 380 U.S. 609, 85 S.Ct. 1229, 14 L.Ed.2d 106 (1965), 493

Griffin v. Illinois, 351 U.S. 12, 76 S.Ct. 585, 100 L.Ed. 891 (1956), 818

Griggs v. Duke Power Co., 401 U.S. 424, 91 S.Ct. 849, 28 L.Ed.2d 158 (1971), 675

Griswold v. Connecticut, 381 U.S. 479, 85 S.Ct. 1678, 14 L.Ed.2d 510 (1965), 537

Grosjean v. American Press Co., 297 U.S. 233, 56 S.Ct. 444, 80 L.Ed. 660 (1936), 860, 1010

Grutter v. Bollinger, 539 U.S. 306, 123 S.Ct. 2325, 156 L.Ed.2d 304 (2003), 701

Guest, United States v., 383 U.S. 745, 86 S.Ct. 1170, 16 L.Ed.2d 239 (1966), 828 
Hall v. Beals, 396 U.S. 45, 90 S.Ct. 200, 24 L.Ed.2d 214 (1969), 82

Hamdan v. Rumsfeld, 548 U.S. 557, 126 S.Ct. 2749, 165 L.Ed.2d 723 (2006), 370

Hamdi v. Rumsfeld, 542 U.S. 507, 124 S.Ct. 2633, 159 L.Ed.2d 578 (2004), 358

Hamdi v. Rumsfeld, 316 F.3d 450 (4th Cir.2003), 369

Hammer v. Dagenhart, 247 U.S. 251, 38 S.Ct. 529, 62 L.Ed. 1101 (1918), 142

Hans v. Louisiana, 134 U.S. 1, 10 S.Ct. 504, 33 L.Ed. 842 (1890), 237

Harper v. Virginia State Bd. of Elections, 383 U.S. 663, 86 S.Ct. 1079, 16 L.Ed.2d 169 (1966), 781,818

Hatfill v. The New York Times Co., 532 F.3d 312 (4th Cir.2008), 929

Heart of Atlanta Motel, Inc. v. United States, 379 U.S. 241, 85 S.Ct. 348, 13 L.Ed.2d 258 (1964), $11,158,841$

Hein v. Freedom From Religion Foundation, Inc., 551 U.S. 587, 127 S.Ct. 2553, 168 L.Ed.2d 424 (2007), 1120

Helvering v. ___ (see opposing party)

Hicklin v. Orbeck, 437 U.S. 518, 98 S.Ct. 2482, 57 L.Ed.2d 397 (1978), 309

Hines v. Davidowitz, 312 U.S. 52, 61 S.Ct. 399, 85 L.Ed. 581 (1941), 246, 816

Hipolite Egg Co. v. United States, 220 U.S. 45, 31 S.Ct. 364, 55 L.Ed. 364 (1911), 141

Hirabayashi v. United States, 320 U.S. 81, 63 S.Ct. 1375, 87 L.Ed. 1774 (1943), 636, 662

Hoke v. United States, 227 U.S. 308, 33 S.Ct. 281, 57 L.Ed. 523 (1913), 141

Holmes v. City of Atlanta, 350 U.S. 879, 76 S.Ct. 141, 100 L.Ed. 776 (1955), 685

Home Bldg. \& Loan Ass'n v. Blaisdell, 290 U.S. 398, 54 S.Ct. 231, 78 L.Ed. 413 (1934), 21, 1238

Hudgens v. N. L. R. B., 424 U.S. 507, 96 S.Ct. 1029, 47 L.Ed.2d 196 (1976), 1285, 1291, 1306

Hughes v. Oklahoma, 441 U.S. 322, 99 S.Ct. 1727, 60 L.Ed.2d 250 (1979), 268

Humphrey's Ex'r v. United States, 295 U.S. 602, 55 S.Ct. 869, 79 L.Ed. 1611 (1935), 12, 427

Hunter v. Underwood, 471 U.S. 222, 105 S.Ct. 1916, 85 L.Ed.2d 222 (1985), 675

Hurley v. Irish-American Gay, Lesbian and Bisexual Group of Boston, 515 U.S. 557, 115 S.Ct. 2338, 132 L.Ed.2d 487 (1995), 1067, 1083

Hurtado v. People of State of California, 110 U.S. 516, 4 S.Ct. 292, 28 L.Ed. 232 (1884), 486, 493

Hustler Magazine v. Falwell, 485 U.S. 46, 108 S.Ct. 876, 99 L.Ed.2d 41 (1988), 936

Illinois ex rel. McCollum v. Board of Educ., 333 U.S. 203, 68 S.Ct. 461, 92 L.Ed. 649 (1948), 1121

Industrial Union Dept., AFLBCIO v. American Petroleum Institute, 448 U.S. 607, 100 S.Ct. 2844, 65 L.Ed.2d 1010 (1980), 397

Ingraham v. Wright, 430 U.S. 651, 97 S.Ct. 1401, 51 L.Ed.2d 711 (1977), 627

In re (see name of party)

I.N.S. v. Chadha, 462 U.S. 919, 103 S.Ct. 2764, 77 L.Ed.2d 317 (1983), 11, 405

Irvin v. Dowd, 366 U.S. 717, 81 S.Ct. 1639, 6 L.Ed.2d 751 (1961), 493

Jackson v. Metropolitan Edison Co., 419 U.S. 345, 95 S.Ct. 449, 42 L.Ed.2d 477 (1974), 1281

Jacobellis v. State of Ohio, 378 U.S. 184, 84 S.Ct. 1676, 12 L.Ed.2d 793 (1964), 937

J.E.B. v. Alabama ex rel. T.B., 511 U.S. 127, 114 S.Ct. 1419, 128 L.Ed.2d 89 (1994), 661

Johnson v. California, 543 U.S. 499, 125 S.Ct. 1141, 160 L.Ed.2d 949 (2005), 692

Jones v. Alfred H. Mayer Co., 392 U.S. 409, 88 S.Ct. 2186, 20 L.Ed.2d 1189 (1968), 824, 1277 
Kahriger, United States v., 345 U.S. 22, 73 S.Ct. 510, 97 L.Ed. 754 (1953), 189

Karcher v. Daggett, 462 U.S. 725, 103 S.Ct. 2653, 77 L.Ed.2d 133 (1983), 796

Kassel v. Consolidated Freightways Corp. of Delaware, 450 U.S. 662, 101 S.Ct. 1309, 67 L.Ed.2d 580 (1981), 279

Katzenbach v. McClung, 379 U.S. 294, 85 S.Ct. 377, 13 L.Ed.2d 290 (1964), 11, 159

Katzenbach v. Morgan, 384 U.S. 641, 86 S.Ct. 1717, 16 L.Ed.2d 828 (1966), 838, 843, 848

Kelo v. City of New London, Conn., 545 U.S. 469, 125 S.Ct. 2655, 162 L.Ed.2d 439 (2005), 1263

Kerrigan v. Commissioner of Public Health, 289 Conn. 135, 957 A.2d 407 (2008), 776

Kirby Forest Industries, Inc. v. United States, 467 U.S. 1, 104 S.Ct. 2187, 81 L.Ed.2d 1 (1984), 1246

Kitzmiller v. Dover Area School Dist., 400 F.Supp.2d 707 (M.D.Pa.2005), 1153

Knowlton v. Moore, 178 U.S. 41, 20 S.Ct. 747, 44 L.Ed. 969 (1900), 633

Kolender v. Lawson, 461 U.S. 352, 103 S.Ct. 1855, 75 L.Ed.2d 903 (1983), 903

Konigsberg v. State Bar of Cal., 366 U.S. 36, 81 S.Ct. 997, 6 L.Ed.2d 105 (1961), 860

Korematsu v. United States, 323 U.S. 214, 65 S.Ct. 193, 89 L.Ed. 194 (1944), 636, 661

Kovacs v. Cooper, 336 U.S. 77, 69 S.Ct. 448, 93 L.Ed. 513 (1949), 878

Krueger v. Mitchell, 112 Wis.2d 88, 332 N.W.2d 733 (Wis.1983), 1249

La Belle Iron Works v. United States, 256 U.S. 377, 41 S.Ct. 528, 65 L.Ed. 998 (1921), 638

Lamb's Chapel v. Center Moriches Union Free School Dist., 508 U.S. 384, 113 S.Ct. 2141, 124

L.Ed.2d 352 (1993), 1107

Lawrence v. Texas, 539 U.S. 558, 123 S.Ct. 2472, 156 L.Ed.2d 508 (2003), 583

Law Students Civil Rights Research Council, Inc. v. Wadmond, 401 U.S. 154, 91 S.Ct. 720, 27

L.Ed.2d 749 (1971), 903, 1060

Lee, United States v., 455 U.S. 252, 102 S.Ct. 1051, 71 L.Ed.2d 127 (1982), 1186

Lee v. Weisman, 505 U.S. 577, 112 S.Ct. 2649, 120 L.Ed.2d 467 (1992), 1086, 1107, 1135, 1157, 1230

Lemon v. Kurtzman, 403 U.S. 602, 91 S.Ct. 2105, 29 L.Ed.2d 745 (1971), 1091, 1101, 1224, 1229

Lewis v. Harris, 188 N.J. 415, 908 A.2d 196 (N.J.2006), 776

Lochner v. New York, 198 U.S. 45, 25 S.Ct. 539, 49 L.Ed. 937 (1905), 515, 729

Locke v. Davey, 540 U.S. 712, 124 S.Ct. 1307, 158 L.Ed.2d 1 (2004), 1219

Lopez, United States v., 514 U.S. 549, 115 S.Ct. 1624, 131 L.Ed.2d 626 (1995), 11, 154, 163

Loretto v. Teleprompter Manhattan CATV Corp., 458 U.S. 419, 102 S.Ct. 3164, 73 L.Ed.2d 868 (1982), 1250

Los Angeles, City of v. Alameda Books, Inc., 535 U.S. 425, 122 S.Ct. 1728, 152 L.Ed.2d 670 (2002), 868

Loving v. United States, 517 U.S. 748, 116 S.Ct. 1737, 135 L.Ed.2d 36 (1996), 397

Loving v. Virginia, 388 U.S. 1, 87 S.Ct. 1817, 18 L.Ed.2d 1010 (1967), 575, 689, 1271

Lucas v. South Carolina Coastal Council, 505 U.S. 1003, 112 S.Ct. 2886, 120 L.Ed.2d 798 (1992), 1259

Lugar v. Edmondson Oil Co., Inc., 457 U.S. 922, 102 S.Ct. 2744, 73 L.Ed.2d 482 (1982), 1272, 1292, 1296, 1306

Lujan v. Defenders of Wildlife, 504 U.S. 555, 112 S.Ct. 2130, 119 L.Ed.2d 351 (1992), 90

Lynch v. Donnelly, 465 U.S. 668, 104 S.Ct. 1355, 79 L.Ed.2d 604 (1984), 1086, 1158, 1230 
Maine v. Taylor, 477 U.S. 131, 106 S.Ct. 2440, 91 L.Ed.2d 110 (1986), 271

Malloy v. Hogan, 378 U.S. 1, 84 S.Ct. 1489, 12 L.Ed.2d 653 (1964), 493

Marbury v. Madison, 5 U.S. 137, 2 L.Ed. 60 (1803), 12, 46, 236

Marriage Cases, In re, 76 Cal.Rptr.3d 683, 183 P.3d 384 (Cal.2008), 591, 776

Marsh v. Chambers, 463 U.S. 783, 103 S.Ct. 3330, 77 L.Ed.2d 1019 (1983), 1138, 1153, 1230

Marsh v. State of Ala., 326 U.S. 501, 66 S.Ct. 276, 90 L.Ed. 265 (1946), 1281, 1306

Martin v. Hunter's Lessee, 14 U.S. 304, 4 L.Ed. 97 (1816), 55

Massachusetts Bd. of Retirement v. Murgia, 427 U.S. 307, 96 S.Ct. 2562, 49 L.Ed.2d 520 (1976), 761

Masson v. New Yorker Magazine, Inc., 501 U.S. 496, 111 S.Ct. 2419, 115 L.Ed.2d 447 (1991), 928

Mathews v. Eldridge, 424 U.S. 319, 96 S.Ct. 893, 47 L.Ed.2d 18 (1976), 628

McAuliffe v. City of New Bedford, 155 Mass. 216, 29 N.E. 517 (Mass.1892), 625, 1001

McCardle, Ex parte, 74 U.S. 506, 19 L.Ed. 264 (1868), 98

McCleskey v. Kemp, 481 U.S. 279, 107 S.Ct. 1756, 95 L.Ed.2d 262 (1987), 678

McConnell v. Federal Election Commission, 540 U.S. 93, 124 S.Ct. 619, 157 L.Ed.2d 491 (2003), 992

McCreary County, Ky. v. American Civil Liberties Union of Ky., 545 U.S. 844, 125 S.Ct. 2722, 162 L.Ed.2d 729 (2005), 1099, 1158, 1169

McDaniel v. Paty, 435 U.S. 618, 98 S.Ct. 1322, 55 L.Ed.2d 593 (1978), 1186

McDonald v. City of Chicago, __ U.S. __, 130 S.Ct. 3020, 177 L.Ed.2d 894 (2010), 494, 580

McIntyre v. Ohio Elections Com'n, 514 U.S. 334, 115 S.Ct. 1511, 131 L.Ed.2d 426 (1995), 937

M'Culloch v. State, 17 U.S. 316, 4 L.Ed. 579 (1819), 110

Medtronic, Inc. v. Lohr, 518 U.S. 470, 116 S.Ct. 2240, 135 L.Ed.2d 700 (1996), 252

Members of City Council of City of Los Angeles v. Taxpayers for Vincent, 466 U.S. 789, 104 S.Ct. 2118, 80 L.Ed.2d 772 (1984), 903

Metro Broadcasting, Inc. v. F.C.C., 497 U.S. 547, 110 S.Ct. 2997, 111 L.Ed.2d 445 (1990), 638

Metropolitan Life Ins. Co. v. Ward, 470 U.S. 869, 105 S.Ct. 1676, 84 L.Ed.2d 751 (1985), 306

Meyer v. Nebraska, 262 U.S. 390, 43 S.Ct. 625, 67 L.Ed. 1042 (1923), 531

Miami Herald Pub. Co. v. Tornillo, 418 U.S. 241, 94 S.Ct. 2831, 41 L.Ed.2d 730 (1974), 1038, 1049

Michael H. v. Gerald D., 491 U.S. 110, 109 S.Ct. 2333, 105 L.Ed.2d 91 (1989), 578

Michael M. v. Superior Court of Sonoma County, 450 U.S. 464, 101 S.Ct. 1200, 67 L.Ed.2d 437 (1981), 746

Miller v. California, 413 U.S. 15, 93 S.Ct. 2607, 37 L.Ed.2d 419 (1973), 937, 941, 942, 1012

Miller v. Johnson, 515 U.S. 900, 115 S.Ct. 2475, 132 L.Ed.2d 762 (1995), 726

Miller, United States v., 307 U.S. 174, 59 S.Ct. 816, 83 L.Ed. 1206 (1939), 37

Milligan, Ex parte, 71 U.S. 2, 18 L.Ed. 281 (1866), 356

Milliken v. Bradley, 433 U.S. 267, 97 S.Ct. 2749, 53 L.Ed.2d 745 (1977), 689

Milliken v. Bradley, 418 U.S. 717, 94 S.Ct. 3112, 41 L.Ed.2d 1069 (1974), 689

Minneapolis \& St. L.R. Co. v. Bombolis, 241 U.S. 211, 36 S.Ct. 595, 60 L.Ed. 961 (1916), 486, 493

Miranda v. Arizona, 384 U.S. 436, 86 S.Ct. 1602, 16 L.Ed.2d 694 (1966), 64

Mississippi University for Women v. Hogan, 458 U.S. 718, 102 S.Ct. 3331, 73 L.Ed.2d 1090 (1982), 745 
Missouri v. Holland, 252 U.S. 416, 40 S.Ct. 382, 64 L.Ed. 641 (1920), 203

Missouri v. Jenkins, 495 U.S. 33, 110 S.Ct. 1651, 109 L.Ed.2d 31 (1990), 689

Mistretta v. United States, 488 U.S. 361, 109 S.Ct. 647, 102 L.Ed.2d 714 (1989), 397

Mitchell v. Helms, 530 U.S. 793, 120 S.Ct. 2530, 147 L.Ed.2d 660 (2000), 1099

M.L.B. v. S.L.J., 519 U.S. 102, 117 S.Ct. 555, 136 L.Ed.2d 473 (1996), 817

Moore v. City of East Cleveland, Ohio, 431 U.S. 494, 97 S.Ct. 1932, 52 L.Ed.2d 531 (1977), 577

Morrison v. Olson, 487 U.S. 654, 108 S.Ct. 2597, 101 L.Ed.2d 569 (1988), 12, 440

Morrison, United States v., 529 U.S. 598, 120 S.Ct. 1740, 146 L.Ed.2d 658 (2000), 173, 846, 1279

Mueller v. Allen, 463 U.S. 388, 103 S.Ct. 3062, 77 L.Ed.2d 721 (1983), 1091, 1107

Muller v. State of Oregon, 208 U.S. 412, 28 S.Ct. 324, 52 L.Ed. 551 (1908), 520, 729

Muskrat v. United States, 219 U.S. 346, 31 S.Ct. 250, 55 L.Ed. 246 (1911), 78

Myers v. United States, 272 U.S. 52, 47 S.Ct. 21, 71 L.Ed. 160 (1926), 423

N. A. A. C. P. v. Button, 371 U.S. 415, 83 S.Ct. 328, 9 L.Ed.2d 405 (1963), 904, 1011

N. A. A. C. P. v. Claiborne Hardware Co., 458 U.S. 886, 102 S.Ct. 3409, 73 L.Ed.2d 1215 (1982), 1054, 1083

N. A. A. C. P. v. State of Ala. ex rel. Patterson, 357 U.S. 449, 78 S.Ct. 1163, 2 L.Ed.2d 1488 (1958), 1061, 1083

National Broadcasting Co. v. United States, 319 U.S. 190, 63 S.Ct. 997, 87 L.Ed. 1344 (1943), 398

National Collegiate Athletic Ass'n v. Tarkanian, 488 U.S. 179, 109 S.Ct. 454, 102 L.Ed.2d 469 (1988), 1300, 1306

National League of Cities v. Usery, 426 U.S. 833, 96 S.Ct. 2465, 49 L.Ed.2d 245 (1976), 206

Near v. State of Minnesota ex rel. Olson, 283 U.S. 697, 51 S.Ct. 625, 75 L.Ed. 1357 (1931), 895, 1015

Nebbia v. People of New York, 291 U.S. 502, 54 S.Ct. 505, 78 L.Ed. 940 (1934), 521

Nebraska Press Ass'n v. Stuart, 427 U.S. 539, 96 S.Ct. 2791, 49 L.Ed.2d 683 (1976), 894

New Energy Co. of Indiana v. Limbach, 486 U.S. 269, 108 S.Ct. 1803, 100 L.Ed.2d 302 (1988), 264, 292

New Orleans, City of v. Dukes, 427 U.S. 297, 96 S.Ct. 2513, 49 L.Ed.2d 511 (1976), 633

New Orleans City Park Improvement Association v. Detiege, 358 U.S. 54, 79 S.Ct. 99, 3 L.Ed.2d 46 (1958), 685

New York v. Ferber, 458 U.S. 747, 102 S.Ct. 3348, 73 L.Ed.2d 1113 (1982), 943, 971, 1012

New York v. United States, 505 U.S. 144, 112 S.Ct. 2408, 120 L.Ed.2d 120 (1992), 213, 794

New York City Transit Authority v. Beazer, 440 U.S. 568, 99 S.Ct. 1355, 59 L.Ed.2d 587 (1979), 643

New York Times Co. v. Sullivan, 376 U.S. 254, 84 S.Ct. 710, 11 L.Ed.2d 686 (1964), 928, 929, 994, 1012

New York Times Co. v. United States, 403 U.S. 713, 91 S.Ct. 2140, 29 L.Ed.2d 822 (1971), 895, 1011

Nguyen v. I.N.S., 533 U.S. 53, 121 S.Ct. 2053, 150 L.Ed.2d 115 (2001), 751

Nixon v. Administrator of General Services, 433 U.S. 425, 97 S.Ct. 2777, 53 L.Ed.2d 867 (1977), 458,459

Nixon v. Fitzgerald, 457 U.S. 731, 102 S.Ct. 2690, 73 L.Ed.2d 349 (1982), 378 
Nixon v. Shrink Missouri Government PAC, 528 U.S. 377, 120 S.Ct. 897, 145 L.Ed.2d 886 (2000), 991

Nixon v. United States, 506 U.S. 224, 113 S.Ct. 732, 122 L.Ed.2d 1 (1993), 72

Nixon, United States v., 418 U.S. 683, 94 S.Ct. 3090, 41 L.Ed.2d 1039 (1974), 372

N.L.R.B. v. Jones \& Laughlin Steel Corp., 301 U.S. 1, 57 S.Ct. 615, 81 L.Ed. 893 (1937), 149

North American Cold Storage Co. v. City of Chicago, 211 U.S. 306, 29 S.Ct. 101, 53 L.Ed. 195 (1908), 628

Northeastern Florida Chapter of Associated General Contractors of America v. City of Jacksonville, Fla., 508 U.S. 656, 113 S.Ct. 2297, 124 L.Ed.2d 586 (1993), 82, 89

North Texas Speciality Physicians v. F.T.C., 528 F.3d 346 (5th Cir.2008), 974

Northwest Austin Mun. Utility Dist. No. One v. Holder, __ U.S.__, 129 S.Ct. 2504, 174 L.Ed.2d 140 (2009), 836

Norwich Gaslight Co. v. Norwich City Gas Co., 25 Conn. 19 (1856), 482

Noto v. United States, 367 U.S. 290, 81 S.Ct. 1517, 6 L.Ed.2d 836 (1961), 1054

O'Brien, United States v., 391 U.S. 367, 88 S.Ct. 1673, 20 L.Ed.2d 672 (1968), 14, 864, 884, 948, 981, 1011, 1012, 1080, 1193

O'Connor v. Donaldson, 422 U.S. 563, 95 S.Ct. 2486, 45 L.Ed.2d 396 (1975), 627

Ogden v. Saunders, 25 U.S. 213, 6 L.Ed. 606 (1827), 1237

Opinion of the Justices to the Governor, 363 Mass. 899, 298 N.E.2d 840 (Mass.1973), 81

Oregon v. Mitchell, 400 U.S. 112, 91 S.Ct. 260, 27 L.Ed.2d 272 (1970), 65

Orr v. Orr, 440 U.S. 268, 99 S.Ct. 1102, 59 L.Ed.2d 306 (1979), 743

O'Shea v. Littleton, 414 U.S. 488, 94 S.Ct. 669, 38 L.Ed.2d 674 (1974), 81

Overton v. Bazzetta, 539 U.S. 126, 123 S.Ct. 2162, 156 L.Ed.2d 162 (2003), 1052

Palko v. Connecticut, 302 U.S. 319, 58 S.Ct. 149, 82 L.Ed. 288 (1937), 486

Palmer v. Thompson, 403 U.S. 217, 91 S.Ct. 1940, 29 L.Ed.2d 438 (1971), 677

Palmore v. Sidoti, 466 U.S. 429, 104 S.Ct. 1879, 80 L.Ed.2d 421 (1984), 692, 705

Parents Involved in Community Schools v. Seattle School Dist. No. 1, 551 U.S. 701, 127 S.Ct. 2738, 168 L.Ed.2d 508 (2007), 712

Paris Adult Theatre I v. Slaton, 413 U.S. 49, 93 S.Ct. 2628, 37 L.Ed.2d 446 (1973), 942

Paul v. State of Virginia, 75 U.S. 168, 19 L.Ed. 357 (1868), 309

Pell v. Procunier, 417 U.S. 817, 94 S.Ct. 2800, 41 L.Ed.2d 495 (1974), 1048

Penn Cent. Transp. Co. v. City of New York, 438 U.S. 104, 98 S.Ct. 2646, 57 L.Ed.2d 631 (1978), 1253

Pennell v. City of San Jose, 485 U.S. 1, 108 S.Ct. 849, 99 L.Ed.2d 1 (1988), 1252

Pennsylvania v. Union Gas Co., 491 U.S. 1, 109 S.Ct. 2273, 105 L.Ed.2d 1 (1989), 238

Pennsylvania Coal Co. v. Mahon, 260 U.S. 393, 43 S.Ct. 158, 67 L.Ed. 322 (1922), 1253, 1258, 1259

Pennzoil Co. v. Texaco, Inc., 481 U.S. 1, 107 S.Ct. 1519, 95 L.Ed.2d 1 (1987), 819

Perry v. Schwarzenegger, 704 F.Supp.2d 921 (N.D.Cal.2010), 776

Perry v. Sindermann, 408 U.S. 593, 92 S.Ct. 2694, 33 L.Ed.2d 570 (1972), 626, 913

Personnel Adm'r of Massachusetts v. Feeney, 442 U.S. 256, 99 S.Ct. 2282, 60 L.Ed.2d 870 (1979), 751 
Philadelphia, City of v. New Jersey, 437 U.S. 617, 98 S.Ct. 2531, 57 L.Ed.2d 475 (1978), 284

Pickering v. Board of Ed. of Tp. High School Dist. 205, Will County, Illinois, 391 U.S. 563, 88 S.Ct. 1731, 20 L.Ed.2d 811 (1968), 1001

Pierce v. Society of the Sisters of the Holy Names of Jesus and Mary, 268 U.S. 510, 45 S.Ct. 571, 69 L.Ed. 1070 (1925), 531

Pike v. Bruce Church, Inc., 397 U.S. 137, 90 S.Ct. 844, 25 L.Ed.2d 174 (1970), 271, 275

Planned Parenthood of Southeastern Pennsylvania v. Casey, 505 U.S. 833, 112 S.Ct. 2791, 120 L.Ed.2d 674 (1992), 553

Pleasant Grove City, Utah v. Summum, __ U.S. __, 129 S.Ct. 1125, 172 L.Ed.2d 853 (2009), 919

Plessy v. Ferguson, 163 U.S. 537, 16 S.Ct. 1138, 41 L.Ed. 256 (1896), 679, 680

Plyler v. Doe, 457 U.S. 202, 102 S.Ct. 2382, 72 L.Ed.2d 786 (1982), 812

Poe v. Ullman, 367 U.S. 497, 81 S.Ct. 1752, 6 L.Ed.2d 989 (1961), 544, 608

Police Dept. of City of Chicago v. Mosley, 408 U.S. 92, 92 S.Ct. 2286, 33 L.Ed.2d 212 (1972), 755

Pollock v. Farmers' Loan \& Trust Co., 158 U.S. 601, 15 S.Ct. 912, 39 L.Ed. 1108 (1895), 65

Posadas de Puerto Rico Associates v. Tourism Co. of Puerto Rico, 478 U.S. 328, 106 S.Ct. 2968, 92 L.Ed.2d 266 (1986), 975, 1012

Powell v. McCormack, 395 U.S. 486, 89 S.Ct. 1944, 23 L.Ed.2d 491 (1969), 71

Presbyterian Church in United States v. Mary Elizabeth Blue Hull Memorial Presbyterian Church, 393 U.S. 440, 89 S.Ct. 601, 21 L.Ed.2d 658 (1969), 1224, 1230

Principality of Monaco v. State of Mississippi, 292 U.S. 313, 54 S.Ct. 745, 78 L.Ed. 1282 (1934), 237

Printz v. United States, 521 U.S. 898, 117 S.Ct. 2365, 138 L.Ed.2d 914 (1997), 219

Prudential Ins. Co. v. Benjamin, 328 U.S. 408, 66 S.Ct. 1142, 90 L.Ed. 1342 (1946), 154, 302

Quirin, Ex parte, 317 U.S. 1, 63 S.Ct. 1, 87 L.Ed. 3 (1942), 357, 365

Railroad Retirement Board v. Alton R. Co., 295 U.S. 330, 55 S.Ct. 758, 79 L.Ed. 1468 (1935), 145

Railway Exp. Agency v. People of State of N.Y., 336 U.S. 106, 69 S.Ct. 463, 93 L.Ed. 533 (1949), 639

R.A.V. v. City of St. Paul, Minn., 505 U.S. 377, 112 S.Ct. 2538, 120 L.Ed.2d 305 (1992), 964, 1012 Raymond U v. Duke University, 91 N.C.App. 171, 371 S.E.2d 701 (N.C.App.1988), 927

Red Lion Broadcasting Co. v. F.C.C., 395 U.S. 367, 89 S.Ct. 1794, 23 L.Ed.2d 371 (1969), 1032, 1049

Reed v. Reed, 404 U.S. 71, 92 S.Ct. 251, 30 L.Ed.2d 225 (1971), 729, 734

Regents of University of California v. Bakke, 438 U.S. 265, 98 S.Ct. 2733, 57 L.Ed.2d 750 (1978), 89,693

Reno v. Condon, 528 U.S. 141, 120 S.Ct. 666, 145 L.Ed.2d 587 (2000), 227

Renton, City of v. Playtime Theatres, Inc., 475 U.S. 41, 106 S.Ct. 925, 89 L.Ed.2d 29 (1986), 865, 1010

Republican Party of Minnesota v. White, 536 U.S. 765, 122 S.Ct. 2528, 153 L.Ed.2d 694 (2002), 873,1011

Reynolds v. Sims, 377 U.S. 533, 84 S.Ct. 1362, 12 L.Ed.2d 506 (1964), 71, 792

Reynolds v. United States, 98 U.S. 145, 25 L.Ed. 244 (1878), 1086, 1186

Rice v. Santa Fe Elevator Corp., 331 U.S. 218, 67 S.Ct. 1146, 91 L.Ed. 1447 (1947), 247 
Richmond, City of v. J.A. Croson Co., 488 U.S. 469, 109 S.Ct. 706, 102 L.Ed.2d 854 (1989), 695

Richmond Newspapers, Inc. v. Virginia, 448 U.S. 555, 100 S.Ct. 2814, 65 L.Ed.2d 973 (1980), 1042, 1049

Roberts v. United States Jaycees, 468 U.S. 609, 104 S.Ct. 3244, 82 L.Ed.2d 462 (1984), 1052, 1066, 1083

Robinson v. California, 370 U.S. 660, 82 S.Ct. 1417, 8 L.Ed.2d 758 (1962), 493

Roe v. Wade, 410 U.S. 113, 93 S.Ct. 705, 35 L.Ed.2d 147 (1973), 546

Rogers v. Lodge, 458 U.S. 613, 102 S.Ct. 3272, 73 L.Ed.2d 1012 (1982), 722, 803

Romer v. Evans, 517 U.S. 620, 116 S.Ct. 1620, 134 L.Ed.2d 855 (1996), 771

Rosenberger v. Rector and Visitors of University of Virginia, 515 U.S. 819, 115 S.Ct. 2510, 132 L.Ed.2d 700 (1995), 918, 919, 1108, 1147

Rosenblatt v. Baer, 383 U.S. 75, 86 S.Ct. 669, 15 L.Ed.2d 597 (1966), 928

Rosenstock v. Board of Governors of University of North Carolina, 423 F.Supp. 1321 (M.D.N.C.1976), 309

Rostker v. Goldberg, 453 U.S. 57, 101 S.Ct. 2646, 69 L.Ed.2d 478 (1981), 754

Roth v. United States, 354 U.S. 476, 77 S.Ct. 1304, 1 L.Ed.2d 1498 (1957), 937, 938, 1012

Rubin v. Coors Brewing Co., 514 U.S. 476, 115 S.Ct. 1585, 131 L.Ed.2d 532 (1995), 976

Rumsfeld v. Forum for Academic and Institutional Rights, Inc., 547 U.S. 47, 126 S.Ct. 1297, 164 L.Ed.2d 156 (2006), 1078, 1083

Rumsfeld v. Padilla, 542 U.S. 426, 124 S.Ct. 2711, 159 L.Ed.2d 513 (2004), 369

Rust v. Sullivan, 500 U.S. 173, 111 S.Ct. 1759, 114 L.Ed.2d 233 (1991), 914, 1011

Saenz v. Roe, 526 U.S. 489, 119 S.Ct. 1518, 143 L.Ed.2d 689 (1999), 309, 484

Salvation Army v. Department of Community Affairs of State of N.J., 919 F.2d 183 (3rd Cir.1990), 1052

San Antonio Independent School Dist. v. Rodriguez, 411 U.S. 1, 93 S.Ct. 1278, 36 L.Ed.2d 16 (1973), 807

San Diego, Cal., City of v. Roe, 543 U.S. 77, 125 S.Ct. 521, 160 L.Ed.2d 410 (2004), 928

Santa Fe Independent School Dist. v. Doe, 530 U.S. 290, 120 S.Ct. 2266, 147 L.Ed.2d 295 (2000), 1147

Santosky v. Kramer, 455 U.S. 745, 102 S.Ct. 1388, 71 L.Ed.2d 599 (1982), 627

Scales v. United States, 367 U.S. 203, 81 S.Ct. 1469, 6 L.Ed.2d 782 (1961), 1053, 1083

Schad v. Borough of Mount Ephraim, 452 U.S. 61, 101 S.Ct. 2176, 68 L.Ed.2d 671 (1981), 909

School Dist. of Abington Tp., Pa. v. Schempp, 374 U.S. 203, 83 S.Ct. 1560, 10 L.Ed.2d 844 (1963), 1090, 1127

School Dist. of City of Grand Rapids v. Ball, 473 U.S. 373, 105 S.Ct. 3216, 87 L.Ed.2d 267 (1985), 1091

Schweiker v. Wilson, 450 U.S. 221, 101 S.Ct. 1074, 67 L.Ed.2d 186 (1981), 529

Scott v. Emerson, 15 Mo. 576 (Mo.1852), 657

Selective Service System v. Minnesota Public Interest Research Group, 468 U.S. 841, 104 S.Ct. 3348, 82 L.Ed.2d 632 (1984), 458

Seminole Tribe of Florida v. Florida, 517 U.S. 44, 116 S.Ct. 1114, 134 L.Ed.2d 252 (1996), 236, 238

Shapero v. Kentucky Bar Ass'n, 486 U.S. 466, 108 S.Ct. 1916, 100 L.Ed.2d 475 (1988), 909 
Shaw v. Reno, 509 U.S. 630, 113 S.Ct. 2816, 125 L.Ed.2d 511 (1993), 723

Sheldon v. Sill, 49 U.S. 441, 8 How. 441, 12 L.Ed. 1147 (1850), 101

Shelley v. Kraemer, 334 U.S. 1, 68 S.Ct. 836, 92 L.Ed. 1161 (1948), 1273, 1288, 1306

Sherbert v. Verner, 374 U.S. 398, 83 S.Ct. 1790, 10 L.Ed.2d 965 (1963), 625, 1188

Shreveport Rate Case, 234 U.S. 342, 34 S.Ct. 833, 58 L.Ed. 1341 (1914), 136

Silkwood v. Kerr-McGee Corp., 464 U.S. 238, 104 S.Ct. 615, 78 L.Ed.2d 443 (1984), 247

Skinner v. Oklahoma, 316 U.S. 535, 62 S.Ct. 1110, 86 L.Ed. 1655 (1942), 533

Slaughter-House Cases, 83 U.S. 36, 21 L.Ed. 394 (1872), 475, 827

Smith v. Goguen, 415 U.S. 566, 94 S.Ct. 1242, 39 L.Ed.2d 605 (1974), 903

South Carolina v. Baker, 485 U.S. 505, 108 S.Ct. 1355, 99 L.Ed.2d 592 (1988), 227

South Carolina v. Katzenbach, 383 U.S. 301, 86 S.Ct. 803, 15 L.Ed.2d 769 (1966), 832, 843, 848

South Carolina State Highway Department v. Barnwell Bros., 303 U.S. 177, 58 S.Ct. 510, 82

L.Ed. 734 (1938), 272

South Carolina, State of v. United States, 199 U.S. 437, 26 S.Ct. 110, 50 L.Ed. 261 (1905), 21

South-Central Timber Development, Inc. v. Wunnicke, 467 U.S. 82, 104 S.Ct. 2237, 81 L.Ed.2d 71 (1984), 292

South Dakota v. Dole, 483 U.S. 203, 107 S.Ct. 2793, 97 L.Ed.2d 171 (1987), 197, 913, 1082

Southeastern Promotions, Ltd. v. Conrad, 420 U.S. 546, 95 S.Ct. 1239, 43 L.Ed.2d 448 (1975), 895

Southern Pac. Co. v. State of Ariz. ex rel. Sullivan, 325 U.S. 761, 65 S.Ct. 1515, 89 L.Ed. 1915 (1945), 275

Spence v. State of Wash., 418 U.S. 405, 94 S.Ct. 2727, 41 L.Ed.2d 842 (1974), 948, 1012

Stafford v. Wallace, 258 U.S. 495, 42 S.Ct. 397, 66 L.Ed. 735 (1922), 138

State Commercial Fisheries Entry Com'n v. Carlson, 65 P.3d 851 (Alaska 2003), 291

State of (see name of state)

Steffel v. Thompson, 415 U.S. 452, 94 S.Ct. 1209, 39 L.Ed.2d 505 (1974), 903

Stenberg v. Carhart, 530 U.S. 914, 120 S.Ct. 2597, 147 L.Ed.2d 743 (2000), 567

Stevens, United States v., __ U.S. __, 130 S.Ct. 1577, 176 L.Ed.2d 435 (2010), 1007

Stone v. Graham, 449 U.S. 39, 101 S.Ct. 192, 66 L.Ed.2d 199 (1980), 1169

Stone v. State of Mississippi, 101 U.S. 814, 25 L.Ed. 1079 (1879), 1237

Strauder v. State of West Virginia, 100 U.S. 303, 25 L.Ed. 664 (1879), 658

Sugarman v. Dougall, 413 U.S. 634, 93 S.Ct. 2842, 37 L.Ed.2d 853 (1973), 758

Supreme Court of New Hampshire v. Piper, 470 U.S. 274, 105 S.Ct. 1272, 84 L.Ed.2d 205 (1985), 309

Swann v. Charlotte-Mecklenburg Bd. of Ed., 402 U.S. 1, 91 S.Ct. 1267, 28 L.Ed.2d 554 (1971), 689

Swift \& Co. v. United States, 196 U.S. 375, 25 S.Ct. 276, 49 L.Ed. 518 (1905), 138

Syracuse Peace Council v. F.C.C., 867 F.2d 654, 276 U.S.App.D.C. 38 (D.C.Cir.1989), 1037

Talley v. California, 362 U.S. 60, 80 S.Ct. 536, 4 L.Ed.2d 559 (1960), 859

Tennessee v. Lane, 541 U.S. 509, 124 S.Ct. 1978, 158 L.Ed.2d 820 (2004), 850

Texas v. Johnson, 491 U.S. 397, 109 S.Ct. 2533, 105 L.Ed.2d 342 (1989), 949, 1012, 1080

Thomas v. Chicago Park Dist., 534 U.S. 316, 122 S.Ct. 775, 151 L.Ed.2d 783 (2002), 861

Thompson v. Western States Medical Center, 535 U.S. 357, 122 S.Ct. 1497, 152 L.Ed.2d 563 (2002), 982 
Thornburgh v. American College of Obstetricians and Gynecologists, 476 U.S. 747, 106 S.Ct. 2169, 90 L.Ed.2d 779 (1986), 553

Tinker v. Des Moines Independent Community School Dist., 393 U.S. 503, 89 S.Ct. 733, 21 L.Ed.2d 731 (1969), 894, 948

Tomic v. Catholic Diocese of Peoria, 442 F.3d 1036 (7th Cir.2006), 1228

Torcaso v. Watkins, 367 U.S. 488, 81 S.Ct. 1680, 6 L.Ed.2d 982 (1961), 1186

Town of (see name of town)

Troxel v. Granville, 530 U.S. 57, 120 S.Ct. 2054, 147 L.Ed.2d 49 (2000), 577

Turner Broadcasting System, Inc. v. F.C.C., 512 U.S. 622, 114 S.Ct. 2445, 129 L.Ed.2d 497 (1994), 869

Twining v. New Jersey, 211 U.S. 78, 29 S.Ct. 14, 53 L.Ed. 97 (1908), 486

United Haulers Ass'n, Inc. v. Oneida-Herkimer Solid Waste Management Authority, 550 U.S. 330, 127 S.Ct. 1786, 167 L.Ed.2d 655 (2007), 297

United States v. ___ (see opposing party)

United States Dept. of Agriculture v. Moreno, 413 U.S. 528, 93 S.Ct. 2821, 37 L.Ed.2d 782 (1973), 770

United States Parole Commission v. Geraghty, 445 U.S. 388, 100 S.Ct. 1202, 63 L.Ed.2d 479 (1980), 82

United States R.R. Retirement Bd. v. Fritz, 449 U.S. 166, 101 S.Ct. 453, 66 L.Ed.2d 368 (1980), 529,642

United States Term Limits, Inc. v. Thornton, 514 U.S. 779, 115 S.Ct. 1842, 131 L.Ed.2d 881 (1995), 11, 22, 314

United States Trust Co. of New York v. New Jersey, 431 U.S. 1, 97 S.Ct. 1505, 52 L.Ed.2d 92 (1977), 1245

Valentine v. Chrestensen, 316 U.S. 52, 62 S.Ct. 920, 86 L.Ed. 1262 (1942), 973

Van Orden v. Perry, 545 U.S. 677, 125 S.Ct. 2854, 162 L.Ed.2d 607 (2005), 1091, 1099, 1176

Varnum v. Brien, 763 N.W.2d 862 (Iowa 2009), 776

Vieth v. Jubelirer, 541 U.S. 267, 124 S.Ct. 1769, 158 L.Ed.2d 546 (2004), 798

Village of (see name of village)

Virginia v. Black, 538 U.S. 343, 123 S.Ct. 1536, 155 L.Ed.2d 535 (2003), 860, 972, 1010

Virginia, United States v., 518 U.S. 515, 116 S.Ct. 2264, 135 L.Ed.2d 735 (1996), 734, 746

Virginia State Bd. of Pharmacy v. Virginia Citizens Consumer Council, Inc., 425 U.S. 748, 96 S.Ct. 1817, 48 L.Ed.2d 346 (1976), 973

Wabash, St. L. \& P. Ry. Co. v. State of Illinois, 118 U.S. 557, 7 S.Ct. 4, 30 L.Ed. 244 (1886), 259, 262

Wallace v. Jaffree, 472 U.S. 38, 105 S.Ct. 2479, 86 L.Ed.2d 29 (1985), 1087, 1128, 1167, 1230

Walz v. Tax Commission of City of New York, 397 U.S. 664, 90 S.Ct. 1409, 25 L.Ed.2d 697 (1970), 1106

Ward v. Rock Against Racism, 491 U.S. 781, 109 S.Ct. 2746, 105 L.Ed.2d 661 (1989), 879, 981, 1011 
Washington v. Davis, 426 U.S. 229, 96 S.Ct. 2040, 48 L.Ed.2d 597 (1976), 672, 1193

Washington v. Glucksberg, 521 U.S. 702, 117 S.Ct. 2258, 138 L.Ed.2d 772 (1997), 606

Watts v. United States, 394 U.S. 705, 89 S.Ct. 1399, 22 L.Ed.2d 664 (1969), 972

Webster v. Reproductive Health Services, 492 U.S. 490, 109 S.Ct. 3040, 106 L.Ed.2d 410 (1989),

553

Weinberger v. Wiesenfeld, 420 U.S. 636, 95 S.Ct. 1225, 43 L.Ed.2d 514 (1975), 745

Wesberry v. Sanders, 376 U.S. 1, 84 S.Ct. 526, 11 L.Ed.2d 481 (1964), 796

West Coast Hotel Co. v. Parrish, 300 U.S. 379, 57 S.Ct. 578, 81 L.Ed. 703 (1937), 523

West Lynn Creamery, Inc. v. Healy, 512 U.S. 186, 114 S.Ct. 2205, 129 L.Ed.2d 157 (1994), 291

West Virginia State Board of Education v. Barnette, 319 U.S. 624, 63 S.Ct. 1178, 87 L.Ed. 1628 (1943), 1127, 1201

Whitman v. American Trucking Associations, 531 U.S. 457, 121 S.Ct. 903, 149 L.Ed.2d 1 (2001), 398

Whitney v. California, 274 U.S. 357, 47 S.Ct. 641, 71 L.Ed. 1095 (1927), 862

Whitney v. Robertson, 124 U.S. 190, 8 S.Ct. 456, 31 L.Ed. 386 (1888), 350

Wickard v. Filburn, 317 U.S. 111, 63 S.Ct. 82, 87 L.Ed. 122 (1942), 155

Williams, United States v., 553 U.S. 285, 128 S.Ct. 1830, 170 L.Ed.2d 650 (2008), 947

Williamson v. Lee Optical of Oklahoma, 348 U.S. 483, 75 S.Ct. 461, 99 L.Ed. 563 (1955), 527, 642

Willson v. Black-Bird Creek Marsh Co., 27 U.S. 245, 7 L.Ed. 412 (1829), 258

Wisconsin v. Yoder, 406 U.S. 205, 92 S.Ct. 1526, 32 L.Ed.2d 15 (1972), 1186, 1194,

Wolf v. People of the State of Colo., 338 U.S. 25, 69 S.Ct. 1359, 93 L.Ed. 1782 (1949), 492

Wolman v. Walter, 433 U.S. 229, 97 S.Ct. 2593, 53 L.Ed.2d 714 (1977), 1099

Wolston v. Reader's Digest Ass'n, Inc., 443 U.S. 157, 99 S.Ct. 2701, 61 L.Ed.2d 450 (1979), 928

Wong Kim Ark, United States v., 169 U.S. 649, 18 S.Ct. 456, 42 L.Ed. 890 (1898), 682

Woods v. Cloyd W. Miller Co., 333 U.S. 138, 68 S.Ct. 421, 92 L.Ed. 596 (1948), 201

Yee v. City of Escondido, Cal., 503 U.S. 519, 112 S.Ct. 1522, 118 L.Ed.2d 153 (1992), 1250

Yerger, Ex parte, 75 U.S. 85, 19 L.Ed. 332 (1868), 101

Yick Wo v. Hopkins, 118 U.S. 356, 6 S.Ct. 1064, 30 L.Ed. 220 (1886), 669

Youngstown Sheet \& Tube Co. v. Sawyer, 343 U.S. 579, 72 S.Ct. 863, 96 L.Ed. 1153 (1952), 11, $328,370,1271$

Zablocki v. Redhail, 434 U.S. 374, 98 S.Ct. 673, 54 L.Ed.2d 618 (1978), 577

Zauderer v. Office of Disciplinary Counsel of Supreme Court of Ohio, 471 U.S. 626, 105 S.Ct. 2265, 85 L.Ed.2d 652 (1985), 909

Zelman v. Simmons-Harris, 536 U.S. 639, 122 S.Ct. 2460, 153 L.Ed.2d 604 (2002), 1110, 1230

Zobel v. Williams, 457 U.S. 55, 102 S.Ct. 2309, 72 L.Ed.2d 672 (1982), 309

Zorach v. Clauson, 343 U.S. 306, 72 S.Ct. 679, 96 L.Ed. 954 (1952), 1121

Zurcher v. Stanford Daily, 436 U.S. 547, 98 S.Ct. 1970, 56 L.Ed.2d 525 (1978), 1023, 1049 


\section{PART I: INTRODUCTION}

\section{ChAPTER 1 \\ History and Overview}

This book concerns the constitutional law of the United States. As you will see in reading it, the book focuses mostly on how the Supreme Court has interpreted various provisions of the U.S. Constitution. This introductory chapter provides some background for understanding the Court's cases. The chapter starts by describing the historical setting in which the Constitution came into being. It then briefly outlines the structure and content of the Constitution. Finally, it presents conflicting theories about how courts should interpret the Constitution.

\section{A. Historical Setting of the Constitution}

In the mid-1700s, the United Kingdom of Great Britain ${ }^{1}$ possessed a number of colonies in North America. Thirteen of these colonies later declared their independence and joined together to form the United States of America: Connecticut, Delaware, Georgia, Maryland, Massachusetts, New Hampshire, New Jersey, New York, North Carolina, Pennsylvania,

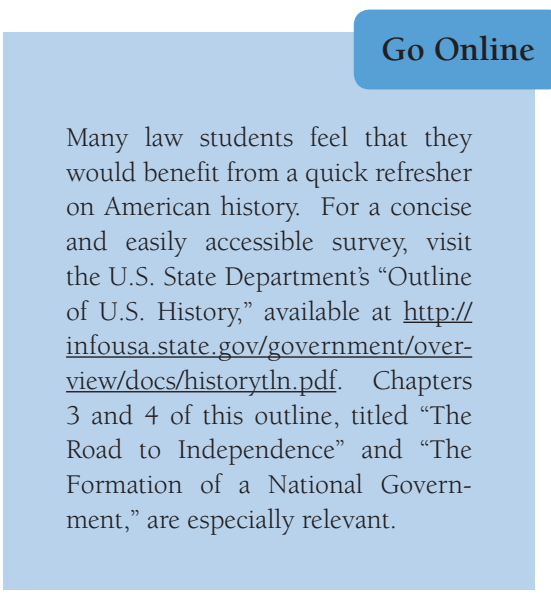

1 "Great Britain" is the name of the large island that England, Scotland, and Wales occupy. The Kingdom of England annexed Wales through Acts of Parliament passed in 1536 and 1543. In 1707, the Act of Union merged the Kingdom of England and the Kingdom of Scotland to form a new nation called the "United Kingdom of Great Britain." Historical discussions often shorten the lengthy name of this nation to the "United Kingdom" or "Great Britain" or just "Britain." 
Rhode Island, South Carolina and Virginia. ${ }^{2}$

What led these colonies to seek independence? What did they hope to accomplish in forming a new nation? How did they organize their government? These are questions that anyone studying the Constitution must consider.

\section{Colonial Governance and Events Preceding the Revolution}

The thirteen colonies that later formed the United States exercised a fair degree of self-governance for many years. Each of the colonies had an elected assembly or legislature, which had authority to pass laws. In addition, these assemblies generally had the sole power to impose taxes and the sole authority to set the budget. The assemblies' enactments were subject to disapproval by a governor (who was appointed by the King of England in all of the colonies except Connecticut and Rhode Island) and by an appointed legislative council (except in Pennsylvania). But actual disapproval of legislation was very rare, and until the mid-1700s, few disputes arose between the colonies and the King or Parliament.

Serious challenges to the colonies' self-governance did not come about until the end of the French and Indian War. In this conflict, which lasted from 1754 until 1763, the United Kingdom fought against France and France's American Indian allies in North America. Although the United Kingdom ultimately prevailed, it incurred enormous expenses in the process. To help recover some of that money, Parliament passed several acts that sought to raise revenue from the colonies. These acts included the Stamp Act of 1765 and Townshend Act of 1767, both of which imposed taxes on various goods within the colonies.

Many colonists believed that these acts exceeded the power of Parliament. They asserted that any taxation imposed on the colonists must come from their own assemblies. Some of the colonists consequently protested the legislation with petitions and civil disobedience. In 1770, to alleviate tensions, Parliament repealed the taxes on almost all goods. But Parliament retained a tax on tea, largely to demonstrate that it did have power to tax the colonies if it so chose.

Colonists responded to the remaining tax by boycotting British tea and by smuggling tea into the country without paying taxes on it. (John Hancock, who later played a key role in the formation of the United States, was indicted on criminal charges for illegally purchasing and reselling tea from Holland.) Parliament in turn passed the Tea Act of 1773, which allowed the English East India Company to import and sell tea at lower prices than what the colonial merchants were charging, with the hope of inducing Americans to stop their boycott of British tea. To thwart this measure, on December 16, 1773, a group called the Sons of Liberty boarded ships in Boston Harbor that were carrying East India Company tea and threw the tea into the water.

${ }^{2}$ In North America, the United Kingdom also possessed the colonies of Quebec, Nova Scotia, Prince Edward Island, East Florida, and West Florida, but these colonies did not seek independence at the time of the American Revolution. Prior to the Revolution, the territory that later became Vermont was not recognized as a separate colony; instead, it was claimed by the colonies of both New York and New Hampshire. Vermont declared its independence from Great Britain and from New York and New Hampshire in 1777, but did not participate in the government formed by the Articles of Confederation. It joined the United States, after ratification of the Constitution, in 1791. Massachusetts assumed control over Maine in the mid-1600s and retained that control until 1820. Maine accordingly did not sign the Declaration of Independence. 
In 1774 , in response to this famous "Boston Tea Party," Parliament passed five laws that the colonists called the "Intolerable Acts" or the "Coercive Acts." These acts, among other things, severely limited the civil and political rights of colonists in Massachusetts. The goal was to force the colonists to make restitution for the tea and generally cease their defiance of Parliament. The founders of the United States remembered the loss of their liberty when they later drafted the Constitution and Bill of Rights.

\section{The First and Second Continental Congresses}

The Intolerable Acts and other factors led concerned colonists from all of the colonies except Georgia to send delegates to a meeting in Philadelphia. The meeting became known as the "First Continental Congress." The delegates met peacefully between September 5 and October 26, 1774. In total, fifty delegates attended. They included many famous figures of the era, such as John Adams, Samuel Adams, Patrick Henry, John Jay, Richard Henry Lee, Peyton Randolph, Roger Sherman, and George Washington.

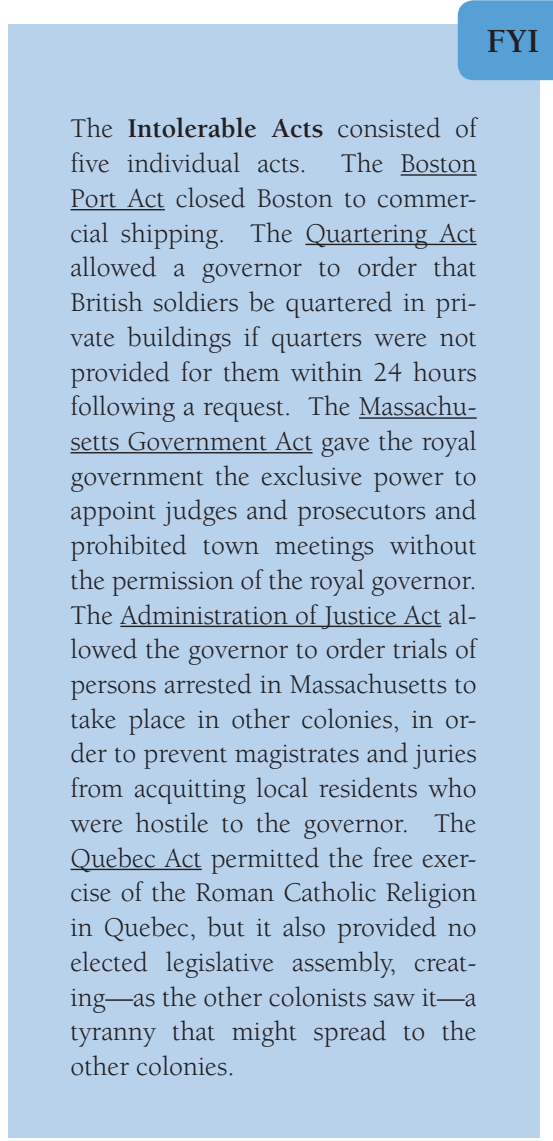

The delegates to the First Continental Con-

gress did not act as if they had the power to pass laws or take any other governmental actions. Instead, the delegates merely adopted resolutions and submitted letters and petitions of grievances to the King. Before adjourning, the First Continental Congress agreed

\section{FYI}

The records of the First and Second Continental Congress are collected and published in the Journals of the Continental Congress, 1774-1789 (Worthington C. Ford et al. eds. 1904-37), the full text of which is available at the Library of Congress's website: http://memory.loc.gov/am$\underline{\text { mem/amlaw/lwjc.html. }}$

to reconvene on May 10, 1775, in Philadelphia.

Before the appointed date for the Second Continental Congress arrived, war broke out in Massachusetts. The fighting started with the battle of Lexington and Concord on April 19, 1775. In that battle, local militiamen defeated British troops who had come to seize their stores of weapons. Following the battle, colonists drove the British troops back to Boston and surrounded the city.

It was in these circumstances that the Second Continental Congress began to meet in Philadelphia on May 10, 1775. The Congress included delegates from each of the thirteen 
colonies. These delegates included many famous men of the founding era, such as John Hancock, John Adams, Benjamin Franklin, George Washington, and Thomas Jefferson. Like the First Continental Congress, the Second Continental Congress had no clear legislative authority. It could adopt resolutions, but could not pass laws or impose taxes.

Nevertheless, on June 15, 1775, the Second Continental Congress decided to assume authority over the American forces surrounding Boston, resolving that "a General be appointed to command all the continental forces, raised, or to be raised, for the defence of American liberty." It then unanimously selected George Washington for this position. The Second Continental Congress also appointed additional subordinate officers, and it agreed to finance the military (although obtaining the funds for this purpose proved difficult). Over the course of the next eleven months, fighting between American and British forces continued.

By the summer of 1776 , the war had convinced the colonists that the colonies could no longer remain a part of the United Kingdom. Accordingly, on July 4, 1776, the Second Continental Congress adopted the Declaration of Independence. With this declaration, the fighting with Great Britain became a war for independence. The war for independence-known now as the "Revolution" or "Revolutionary War"-lasted seven years. The American forces lost most of the battles, but held together and eventually prevailed. In 1783, the United Kingdom acknowledged the independence of the United States in the Treaty of Paris, and the war ended.

\section{The Articles of Confederation and the Calling of a Constitutional Convention}

The Declaration of Independence proclaimed that the colonies had become "free and independent states." But these independent states needed to work together to prevail against the United Kingdom. In 1777, while the Revolutionary War was still being fought, the Continental Congress drafted a document called the Articles of Confederation. This document was finally approved by all of the states in 1781 .

The Articles of Confederation was more like a multilateral treaty among allies than a formal constitution for a new national government. The document announced that its purpose was to create a "firm league of friendship" among the former colonies, which had now become something more akin to separate states. It emphasized that each "state retains its sovereignty, freedom, and independence."

\section{Food for Thought}

The newly independent states did not create a strong national government in the Articles of Confederation. Why might they have felt reluctant to do that even though the Revolutionary War clearly showed that the states needed to work together?
Under the Articles of Confederation, the states would continue to send delegates to a Congress just as they had done before. The Congress had limited power to pass laws, with each state having one vote. But the government was not effective. All measures required the unanimous assent of the states. The Congress had no way to enforce laws or collect taxes. There were no national courts. In addition, the unity of the states became strained over trade and other issues. 
In 1787, the weaknesses in the Articles of Confederation led the Congress to call for a convention "for the sole and express purpose of revising the Articles of Confederation and reporting to Congress and the several legislatures such alterations and provisions therein as shall when agreed to in Congress and confirmed by the states render the federal constitution adequate to the exigencies of Government \& the preservation of the Union." Notwithstanding the Congress's mandate, the convention quickly adopted as its task the drafting of a new Constitution. Accordingly, the convention became known as the "Constitutional Convention of 1787" (or sometimes the "Philadelphia Convention" or the "Federal Convention").

The states each could send as many "deputies" as they wanted to the Constitutional

FYI
Although the Constitutional Con-
vention met in secret and the mem-
bers agreed not to discuss what took
place, we now know a great deal
about what transpired at the pro-
ceedings. The Convention appoint-
ed a secretary, who kept an official
journal. In addition, at least eight of
the fifty-five members took notes at
the Convention. By great fortune,
James Madison, who was the intel-
lectual leader of the delegates, took
the most extensive notes. The re-
cords and notes are collected in The
Records of the Federal Convention of
1787 (Max Farrand, ed., 1911), a
source cited by more than 100 Su-
preme Court cases. The Library of
Congress's website contains the full
text of this work: http://memory.loc.
gov/ammem/amlaw/lwfr.html.
Convention. At the Convention, however, the delegates decided that each state would have only one vote. In total, fifty-five men attended the Convention. These men represented all of the states except Rhode Island, which chose not to participate. The deputies, whom we now call the "Framers" of the Constitution, included grand eminences such as George Washington and Benjamin Franklin; visionary political thinkers such as James Madison, Alexander Hamilton, James Wilson, and Roger Sherman; and masters of written expression such as Gouverneur Morris, who did most of the final stylistic editing of the Constitution. The deputies also included dissenters, such as George Mason of Virginia, who refused to vote for the document because it did not contain a bill of rights, and Robert Yates and John Lansing, Jr., who left the Convention early because they believed that their instructions from the New York legislature did not permit them to participate in creating a new constitution.

\section{Proceedings of the Constitutional Convention}

As the cases in this book will show, in interpreting the Constitution, the Supreme Court often looks very carefully at what the deputies argued and decided at the Constitutional Convention. ${ }^{3}$ To understand the Court's frequent references to the deputies' debates, readers of the Court's opinions should know what happened in Philadelphia. Basically, the Convention took place in nine chronological segments:

1. Full Convention (May 14 - May 29). The Convention began by unanimously selecting George Washington to serve as the Convention's president. It then adopted rules gov-

\footnotetext{
${ }^{3}$ As discussed in Part $\mathrm{C}$ of this Chapter, many writers disagree about whether it is possible to discern the original meaning of the Constitution from these debates and, in any event, whether the original meaning should govern modern interpretation of the Constitution.
} 
erning the proceedings. These rules specified, among other things, that each state present and fully represented would have one vote and that the proceedings would be kept secret.

On May 29, 1787, Edmund Randolph offered 15 resolutions, each just one sentence in length. These resolutions—which became known as the "Virginia Plan" for governmentreflected the ideas of James Madison. Under the plan, there would be a national legislature that would have two chambers, one directly elected and the other appointed by the state legislatures. The plan generally favored the states with large populations (Massachusetts, Pennsylvania, New York, and Virginia) because it called for proportional representation in both houses. After Randolph made this proposal, the Convention decided to deliberate as a committee of the whole (i.e., a gathering where all of the deputies could discuss the issues under informal rules of debate).

2. Committee of the Whole (May 30 - June 13). On the first day when the deputies met as a committee of the whole, Gouverneur Morris urged Randolph to modify his resolutions to include the following proposal: "Resolved, that a national government ought to be established consisting of a supreme Legislature, Executive, and Judiciary." The committee of the whole voted to adopt this resolution. With this action, the committee of the whole implicitly endorsed creating a new Constitution as the goal of the Convention, rather than merely amending the Articles of Confederation.

3. Full Convention (June 13 - June 15). The Convention formally debated the Virginia Plan from June 13 to June 15. The small states opposed the Virginia Plan because they believed that their votes would be diluted in a national legislature with proportional representation. On June 15, William Paterson of New Jersey proposed a set of nine alternative resolutions, which became known as the "New Jersey Plan." The New Jersey Plan favored small states. Most significantly, the plan called for a unicameral legislature with equal representation for each state. The Convention again decided to meet as a committee of the whole, this time to discuss the New Jersey Plan.

4. Committee of the Whole (June 16 - June 19). The committee of the whole debated and ultimately rejected the New Jersey Plan. It also considered an alternative plan proposed by Alexander Hamilton. Hamilton's plan called for an executive elected for life and senators chosen for life. The plan would have reduced state sovereignty by allowing the national executive to appoint executives for each state government.

5. Full Convention (June 19 - July 26). Important debates about representation in the legislative branch followed the rejection of the New Jersey Plan. The large and small states could not agree on the composition of the legislative branch. Ultimately, a modified version of the Virginia Plan became acceptable to the Convention after the delegates agreed to what has become known as the "Great Compromise" (or alternatively as the "Connecticut Compromise"). In this compromise, the states would have equal representation in the Senate and proportional representation in the House. This compromise balanced the interests of large and small states. The Convention also adopted the closely related three-fifths rule, under which only three-fifths of a state's slave population would be counted for determining representation in the House. This feature split the difference between Southern states, which wanted all slaves counted for this purpose, and Northern states, which opposed 
counting any slaves in determining state entitlement to representation in the House.

In addition to the Great Compromise, the Convention also addressed a variety of other important topics. These included the term of the executive's service, the appointment of judges, and the process for ratifying the Constitution. On July 26th, with the general structure of the government settled, the Convention created a "Committee of Detail" to turn the plan into a draft. The Convention recessed while the Committee of Detail performed its work.

6. Committee of Detail (July 27 - August 5). John Rutledge, Edmund Randolph, and James Wilson did most of the work of the Committee of Detail. In addition to describing the government and the selection of its members, the Committee of Detail added the list of congressional powers and the list of limitations on state powers now found in Article I. When the Committee of Detail finished, its draft was printed and distributed to all of the deputies.

7. Full Convention (August 6 - September 6). The Full Convention then debated the Committee of Detail's draft and other important matters. They considered suffrage qualifications, immigration, slavery, and the veto power. During this time, the Convention referred some matters to separate committees, which met and reported back to the Convention. After reaching final conclusions on most items, the Convention appointed a "Committee of Style" (sometimes called the "Committee on Style and Arrangement"). The Committee of Style was charged with the task of putting the Constitution in a consistent form.

8. Committee of Style (September 6 - September 12). The Committee of Style put all of the Convention's changes into the draft and polished the text. Gouverneur Morris did much of the work, although the Committee also included James Madison, William Johnson, Rufus King, and Alexander Hamilton. Their work is seen as very important because they formulated the precise expression of many of the Constitution's great clauses. The Committee of Style finished its assignment on September 12, and a printer made copies of its draft for all of the deputies.

9. Full Convention (September 12 - September 17). The Convention then debated the Committee of Style's draft for several days. During this time, George Mason and Elbridge Gerry proposed the inclusion of a bill of rights. The Convention debated but rejected this proposal. Two days later, on September 17, 1787, the state delegations present at the Convention unanimously approved the Constitution. All of the individual delegates present except for Gerry, Mason, and Randolph signed the Constitution. The Convention then adjourned.

\section{Ratification of the Constitution}

The Constitution, by its own terms, could not go into effect until ratified. Article VII said: "The Ratification of the Conventions of nine States, shall be sufficient for the Establishment of this Constitution between the States so ratifying the Same." Under this provision, each state legislature was expected to form a ratifying convention to debate and vote on the Constitution. In the fall of 1787 and spring and early summer of 1788 , the states held these ratifying conventions. 
Whether the proposed Constitution would be ratified was an open question. The Constitution would make many substantial changes to the status quo. Although some people favored ratification, many others did not. Public debate spread throughout the states on the subject. Supporters of the Constitution became known as the "Federalists," while opponents became known as the "Anti-Federalists."

Three of the best known Federalists were Alexander Hamilton, James Madison, and John Jay. In the fall of 1787 and spring of 1788 , they wrote a series of 85 essays explaining the Constitution and urging its ratification in the State of New York. Each of these essays was titled "The Federalist" followed by a number designating its order in the series. Historians typically refer to the 85 essays as the "Federalist Papers." These essays, all of which are available online, address nearly every aspect of the Constitution. Although the essays are advocacy documents and not dispassionate legal analyses, they have been remarkably influential. As you will see in reading the cases in this book, the Supreme Court regularly relies on these essays in attempting to discern the original meaning of

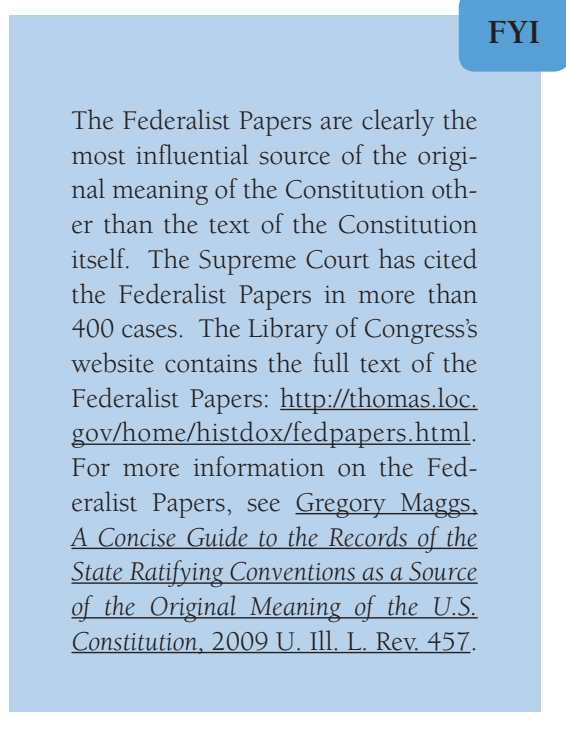
the Constitution.

The Anti-Federalists opposed the Constitution for a number of reasons. Two of the most important concerned the protection of state sovereignty and individual rights. Opponents felt that the Constitution gave too much power to the federal government at the

\section{FYI} expense of the states. They also worried that the Constitution did not contain a bill of rights that would limit the powers of the government. In

Many records from the state ratifying conventions are collected in The Debates in the Several State Conventions on the Adoption of the Federal Constitution, As Recommended by the General Convention at Philadelphia, in 1787 (Jonathan Elliot, ed. 1836-59). This work, often called Elliot's Debates for short, has been very influential; the Supreme Court has cited it in over 100 cases. The full text is available online at the Library of Congress's website: http://memory. loc.gov/ammem/amlaw/lwed.html. thinking about these opponents of the Constitution, modern readers should remember that their concerns stemmed from having lived through Parliament's oppression of the colonies.

By the summer of 1788 , conventions in nine states had approved the Constitution, putting it into effect among the ratifying states. As the following table indicates, ratification was uncontroversial in Delaware, New Jersey, and Georgia. Each of these small states unanimously ratified the document. 
But the Constitution was much more controversial in other states. Indeed, switching just a few votes in big states like New York or Virginia might have scuttled the entire project.

\begin{tabular}{|c|c|c|}
\hline State & $\begin{array}{c}\text { Date of } \\
\text { Ratification }\end{array}$ & Vote \\
\hline Delaware & Dec. 7, 1787 & 30-0 \\
\hline Pennsylvania & Dec. 12, 1787 & $46-23$ \\
\hline New Jersey & Dec. 18, 1787 & $38-0$ \\
\hline Georgia & Jan. 2, 1788 & 26-0 \\
\hline Connecticut & Jan. 9, 1788 & $128-40$ \\
\hline Massachusetts & Feb. 6, 1788 & $187-168$ \\
\hline Maryland & Apr. 28, 1788 & $63-11$ \\
\hline South Carolina & May 23, 1788 & $149-73$ \\
\hline New Hampshire & Jun. 21, 1788 & $57-47$ \\
\hline Virginia & Jun. 25, 1788 & $89-79$ \\
\hline New York & Jul. 26, 1788 & $30-27$ \\
\hline North Carolina & Nov. 21, 1789 & $195-77$ \\
\hline Rhode Island & May 29, 1790 & $34-32$ \\
\hline
\end{tabular}

\section{The First Congress}

Federal elections took place during the fall of 1788, and the new government under the Constitution began in 1789. George Washington, of course, became the first President. In the First Congress, which met from March 1789 to March 1791, a total of 29 persons served as senators and 66 served as representatives.

\section{FYI}

Because the First Congress laid so much of the foundation for the new nation, the Supreme Court often looks to its acts for guidance in determining the original meaning of the Constitution. The acts of the First Congress are published in volume 1 of The Public Statutes at Large of the United States of America (1845), the citation of which is abbreviated as "1 Stat." The Library of Congress has the full text at its website: http://memory.loc.gov/ ammem/amlaw/lwsl.html.
Many of these senators and representatives justifiably could consider themselves experts on the Constitution. Ten of the senators and eleven of the representatives had served as deputies at the Constitutional Convention. Some of them, including James Madison, Oliver Ellsworth, and Roger Sherman, had played prominent roles in the Constitution's drafting. Other members of the First Congress, such as Richard Henry Lee, had participated at state ratifying conventions even though they had not attended the Constitutional Convention. 
During its two-year term, the First Congress passed an astounding 96 acts. Among its many other accomplishments, the First Congress:

- imposed taxes on imported goods and on vessels entering United States ports, providing the first source of federal revenue;

- shaped the executive branch by establishing the Departments of Foreign Affairs, War, and Treasury;

- created the federal judicial system;

- passed laws on naturalization, patents, copyrights, and other subjects still governed by federal law;

- established a system of lighthouses, the post office, and the Bank of the United States;

- provided for the assumption of state revolutionary war debts and paying of the national debt; and

- located the seat of government in the District of Columbia and admitted Kentucky and Vermont into the Union.

- The First Congress also proposed twelve amendments to the Constitution, ten of which (now known as the Bill of Rights) received quick ratification by the states.

\section{B. Organization of the Constitution}

The Constitution contains a number of different parts. No one expects a new student of constitutional law to commit the document to memory or to master its provisions in one reading. But before going further, you should take some time to become familiar with its organization.

\section{Preamble}

The Constitution starts with an introduction, or "Preamble," which lists the six goals of the People in adopting the Constitution: "to form a more perfect Union, establish Justice, insure domestic Tranquility, provide for the common defence, promote the general Welfare, and secure the Blessings of Liberty to ourselves and our Posterity." Although lofty in tone, the courts mostly have concluded that the language of the Preamble is precatory. In other words, it does not create legal rights, duties, or powers. But the Preamble is still important. For example, it makes clear that the "People" rather than the states adopted the Constitution. We will consider this distinction in cases about the nature of the United States' sovereignty.

\section{Article I}

Following the Preamble, the Constitution contains seven articles. The first three arti- 
cles reflect the Framers' vision that there are three branches of government: the legislative branch, the executive branch, and the judicial branch. Each has its own powers and has some ability to provide checks on the others. But as we will see in Chapter 6 , sometimes questions arise about whether one branch is attempting to exercise powers belonging to another branch.

Article I contains ten sections that address the legislative branch. Section 1 establishes the fundamental point that "[a]ll legislative Powers herein granted shall be vested in a Congress of the United States, which shall consist of a Senate and House of Representatives." This means, among other things, that Congress has these powers, and that the President and the courts do not. You will read about this provision in famous cases like Youngstown Sheet \& Tube v. Sawyer, 343 U.S. 579 (1952), in which the Court held that the President was unconstitutionally attempting to assert powers that were legislative in nature.

Sections 2 through 6 then tell how senators and representatives are selected, what their qualifications must be, how they are paid, and so forth. These provisions come up in the important case of U.S. Term Limits v. Thornton, 514 U.S. 779 (1995), a case invalidating an attempt to add what amounted to additional restrictions on who could run for the House or Senate.

Section 7 describes the procedure that Congress must follow in order to pass a law. As most high school civics classes teach, the House and Senate have to approve a bill, and the President must sign it. If the President vetoes a bill (i.e., rejects it), the House and Senate can override the veto with a two-thirds vote. But we will see in INS v. Chadha, 462 U.S. 919 (1983), that Congress has sometimes been tempted to look for ways to exercise power without involving the President.

Section 8 then lists the subjects upon which Congress may pass laws. It says that Congress may collect taxes, regulate commerce, establish a post office, and so forth. Chapter 3 of this book covers what Congress may and may not do under Article I, section 8. We will see in Heart of Atlanta Motel v. United States, 379 U.S. 241 (1964), and Katzenbach v. McClung, 379 U.S. 294 (1964), that Congress has the power to pass legislation protecting civil rights. On the other hand, we will see in United States v. Lopez, 514 U.S. 549 (1995), and New York v. United States, 505 U.S. 144 (1992), that the Supreme Court has struck down laws attempting to ban guns from schools or order the states to provide for the disposal of radioactive waste as being beyond the power of Congress to enact.

Sections 9 and 10 state various specific prohibitions. Section 9 says that Congress generally cannot suspend the writ of habeas corpus, pass ex post facto laws, tax exports from states, or give preference to one state's ports. Section 10 then tells us some of the things that the states may not do. They cannot enter treaties or coin money, impose duties on imports and exports, and so forth.

\section{Article II}

Article II concerns the executive branch. The Chief Executive, of course, is the President of the United States. Article II, section 1 says: "The executive Power shall be vested in a President of the United States of America." But the President does not act alone. The 
federal departments and agencies assist the President. The Constitution does not create these departments, but in various provisions it contemplates their existence. Congress has created many such departments, including the Department of Justice, the Department of Defense, the State Department, and so forth.

The President is the chief executive. In general, that makes everyone else in the executive branch subordinate to the President. The President generally exercises control by firing or threatening to fire those who will not carry out his lawful policies. But we will see in cases like Humphrey's Executor v. United States, 295 U.S. 602 (1935), and Morrison v. Olson, 487 U.S. 654 (1988), that Congress may place some limits on the ability of the President to control subordinates in this manner.

Section 2 puts the President in charge of the military, and allows him to grant pardons and appoint judges and other office holders. Section 3 requires the President to report to Congress, receive ambassadors, make sure that the laws are faithfully executed, and so forth.

\section{Article III}

Article III describes the power of the federal judiciary. The first sentence of Section 1 tells us that "[t]he judicial Power of the United States, shall be vested in one supreme Court, and in such inferior Courts as the Congress may from time to time ordain and establish." Section 2 then tells about the subject matter jurisdiction of federal courts. For example, it says that they can hear lawsuits between "Citizens of different States," which we know as diversity jurisdiction. We will see in the first case included in Chapter 2, Marbury v. Madison, 5 U.S. (1 Cranch) 137 (1803), that the Court struck down a federal statute that attempted to give the Court jurisdiction beyond the limits of Article III, section 2.

\section{Articles IV - VII}

Articles IV through VII contain a variety of different provisions. Article IV generally addresses relations among the states. As we will see in Chapter 4, the Privileges and Immunities Clause in Article IV limits discrimination by states against citizens of other states. Article V discusses the amendment process. The House and Senate can propose amendments by a two-thirds vote or two-thirds of the states can call a convention. (The latter route for amendment has never been used.) Proposals for amending the Constitution become effective when three-fourths of the states have ratified them in their legislatures or in conventions. Article VI tells us, among other things, that the "Constitution, and the Laws of the United States *** shall be the supreme Law of the Land." Article VII describes the ratification process that had to occur before the Constitution could take effect.

\section{Amendments}

The Constitution now contains 27 amendments. The first 10 amendments, commonly called the Bill of Rights, protect a large number of individual rights. Amendments 13 through 15 are known as the "Civil War Era Amendments" or the "Reconstruction Amendments" because they were passed at the end of the Civil War during the process of Reconstruction. They abolish slavery, bar states from denying equal protection of the laws 
or due process of the law to any person, and protect voting rights. We will be looking extensively at the First Amendment's protection of Free Speech, Free Press, and Religion, and at the Fourteenth Amendment's guarantees of Equal Protection and Due Process, in the second half of this book.

\section{Methods of Interpreting the Constitution}

One of the many things that distinguished the American Constitution at the time of its ratification was the simple fact that it was written down. (Although the United Kingdom - the nation from which the United States broke but from which it inherited its legal tradition-also had a "constitution," it was developed over time, in a common-law fashion, rather than codified.) At first blush, one might expect there to be little need for a thick casebook of judicial opinions for matters governed by a written document. But the study of American constitutional law has, from the very beginning, been as much a study of judicial interpretations of the Constitution as it has been of the document itself. We will see one of the reasons why this is so in Chapter 2 when we consider Marbury v. Madison and the topic of judicial review. But answering the question of who should get to interpret the Constitution-a question on which there is substantial continuing debate, as we will see in Chapter 2-does not tell us why a written Constitution should so frequently require interpretation in the first place. As it turns out, there has long been a need for interpretation of the Constitution because it is often not obvious, even after careful consultation of the text of the Constitution, what the Constitution tells us about important questions.

There are several reasons why this is so. First, the Framers of the Constitution (and those who ratified it) sought to preserve some degree of flexibility for subsequent generations to address pressing problems and, if necessary, to structure the government accordingly. Second, as described above, although the Framers were able to achieve consensus by compromising on some controversial questions, they masked their disagreement on other questions by writing general and vague provisions. Third, the Framers simply did not anticipate some of the questions that would arise in the future. Today's problems often look very different from the problems of 1789_or 1868, when the Fourteenth Amendment was ratified. Accordingly, the plain text of the Constitution often does not speak directly to questions that are likely to arise today.

One possible response to constitutional silence would be to conclude that if the Constitution does not expressly prohibit a particular action, it permits it. The Constitution, after all, is almost entirely about the limits on governmental, rather than private, actionthe sole (and obviously incredibly important) exception being the Thirteenth Amendment, which prohibits slavery - and one could sensibly conclude that if the Constitution does not prohibit the government from taking a certain action, it implicitly permits it. There is great appeal to this approach; indeed, as we will see, among other things, it attempts to preserve democratic government by preventing judicial interference with the modern choices of democratically elected officials. But even if one is persuaded that it is the proper response 
to constitutional silence, it does not help us to determine when in fact the Constitution is silent on a particular question. This is because the Constitution's broad provisions arguably touch on a wide range of topics, even though they fail to provide specific guidance on most controversial questions that are likely to arise.

Indeed, the Constitution tends to speak at very high levels of generality. There is little doubt, for example, that the First Amendment protects the freedom of speech, but it does not define "freedom" or "speech." Is a contribution to a candidate for public office a form of "speech"? See Buckley v. Valeo, 424 U.S. 1 (1976), which we will consider in Chapter 14. What about publicly burning a draft card as a form of protest against an ongoing war? See United States v. O’Brien, 391 U.S. 367 (1968), which we will also consider in Chapter 14. And even if we can agree on what counts as speech, does a law imposing some limits on the ability to engage in a particular form of speech—but not prohibiting it entirely"abridg[e]" the "freedom" protected by the Amendment? Similarly, the Fourteenth Amendment prohibits the States from denying to persons the "equal protection of the laws," but it does not give any more guidance about what those terms mean-whether, for example, the government is ever permitted to distinguish among citizens and, if so, whether some bases for classification are more problematic than others; and, if so, whether a State can justify a particular suspect classification-such as a requirement that drivers over 70 years old, but not younger drivers, get annual eye exams, or a ban on women serving in combat in the military-with sufficiently compelling reasons. Even determining whether the Constitution is "silent," therefore, requires interpretation of the document's broad terms.

Of course, the Constitution is not unusual among written texts in requiring interpretation to determine its meaning. Courts routinely are called upon, for example, to interpret statutes and contracts, written texts that only sometimes speak with great detail, and often speak in broad generalities-or, with respect to issues that are litigated, are entirely silent. Although interpretation of such documents is commonplace, there are a range of interpretive approaches that courts follow-and substantial debate over which are appropriate and defensible. In the context of statutory interpretation, for example, there are lively debates over whether judges should consider legislative history to determine statutory meaning, or whether courts should seek to discern congressional purposes in order to shed light on how Congress would have chosen to address particular circumstances about which the statute is silent. Similarly, there is a long-standing debate over whether, in interpreting a contract, courts should consider only the plain meaning of the contract's terms or instead may consider extrinsic evidence to determine a contract's meaning.

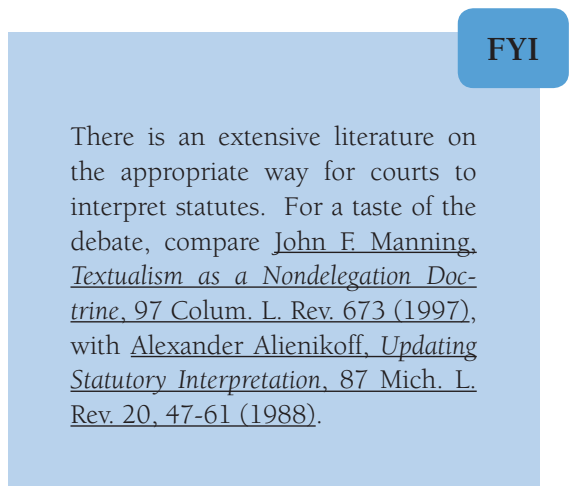

But even assuming that there is a "correct" way to interpret statutes and contracts, matters are at least arguably more complex when the document at issue is the Constitution, because the Constitution is quite different from a statute or a contract in many important 
ways. First, as we will see when we consider Marbury v. Madison in Chapter 2, the Constitution is understood to be a form of "higher" law-that is, it cannot be superseded by an ordinary statute enacted by Congress, and (by its own express terms, in the Supremacy Clause of Article VI) it trumps state law that is inconsistent with its provisions. Second, by its own terms (in Article V), the Constitution can be amended only after obtaining a supermajority consensus-a two-thirds majority in both Houses of Congress and ratification by three-quarters of the States- that has rarely been achieved in over two hundred years. As noted above, there have been only 27 Amendments, and even that number tends to overstate the ease with which the Constitution may be amended: the ten Amendments in the Bill of Rights were adopted together two years after ratification of the original document, and one other (the Twenty-Seventh) was proposed along with the original Bill of Rights; three Amendments were adopted within only a few years after the Civil War; and two of the Amendments— the Eighteenth, enshrining Prohibition, and the Twenty-First, repealing it-effectively cancel each other out. Indeed, not only is it difficult to amend the Constitution, but Article $\mathrm{V}$ actually states that some of its provisions cannot be amended at all, even with the requisite support of two-thirds of both Houses of Congress and three-fourths of the states. Perhaps more strikingly, the provision that cannot be amended today is the one that gives each state an equal voice in the Senate, even though that provision arguably is the single most anti-democratic provision in the Constitution itself. Third, and particularly important in light of the first two distinctive features, the Constitution is over 200 years old.

\section{Food for Thought \\ The provision in the Constitution providing the requirements for its ratification itself was inconsistent with the "constitution" that was in force at the time it was adopted. Al- though Article VII provided that the Constitution would become effective upon ratification by nine of the thir- teen states, the Articles of Confeder- ation expressly required unanimous consent of the states for any amend- ments to its terms. What does this suggest about the extent to which we should treat the current Constitution as binding?}

Together, these features of the Constitution-its status as higher law, the difficulty of amending it, and its age-stand in uneasy tension with the notion of democracy. Indeed, the very notion of constitutionalism means that democratically elected majorities today cannot decide to govern themselves in the manner of their choosing if their choices would conflict with the Constitution. This tension is generally known as the "dead-hand problem": to embrace constitutionalism is to accept that the men who wrote and ratified the Constitution to govern them over 200 years ago should also be able to reach into the future, with their now-dead hands, and tell us how to live our lives.

One possible response to the dead-hand problem, of course, would be to deny the continuing binding force of the Constitution. It is, after all, merely a collection of words with no independent force beyond our willingness to follow it. But this response historically has held little appeal, because each successive generation has seen great value in binding itself to a charter for self-governance. First, it would be difficult for government to function without rules for the proper exercise of its power. Second, there is good reason to limit the 
power of democratic majorities. The Constitution is a form of self-imposed paternalism, to prevent us from letting the perceived exigencies of the moment lead us to decisions that, upon reflection and with the clarity of hindsight, we know are destructive of our most deeply held values.

And, as explained above, if we are to embrace the Constitution, then we must have some way to interpret it. The debate over how to interpret the Constitution is at least as old as the Constitution itself and tends to reflect the basic tension created by the very notion of constitutionalism. On the one hand, if we accept that the Constitution is a form of law (albeit higher law), then there is a strong argument that we should interpret it as we would any other law-to have a generally fixed content, determined by the will of those who enacted it, embodied either in their intentions, in the text itself, or in the understanding of that text at the time it was enacted. Indeed, one could forcefully argue that if the Constitution did not mean what its Framers thought it meant-or at least something closely approximating, at some level of generality, what they thought it meant-then the Constitution would not truly be a form of law in any conventional sense. This conception of the proper way to interpret the Constitution forms the theoretical basis of the approach to interpretation known as "originalism." Proponents of originalism argue that a provision of the Constitution must mean today what it meant, or perhaps was understood to mean, when it was ratified.

On the other hand, if we are concerned about the dead-hand problem and its seeming inconsistency with our democratic impulses today, then we might seek to interpret the Constitution in a way that reflects values that have enduring support, and not simply those that were important at the time of the Founding. According to this view, if the Constitution is binding principally because we agree to be bound by it, and we are eager to be bound by it only if we perceive it to be legitimate according to contemporary values, then we must update the Constitution in order to preserve it. This conception of the proper way to interpret the Constitution is often called "non-originalism," defined in contrast to what it is not. Most non-originalists rely to some degree on the original meaning of the Constitution, at least at a high level of generality, but also see room for constitutional meaning to evolve.

The debate over these competing views reflects the fundamental paradox of constitutionalism: if the Constitution is a form of law, then its meaning to the people who adopted it must be central to its meaning today. But do those people have a right to bind us to their choices? In the sections that follow, we consider the conventional arguments for and against originalism and non-originalism.

\section{Originalism}

Originalism is an approach to interpretation that accords dispositive weight to the original meaning of the Constitution. According to this approach, a provision of the Constitution must mean today what it meant when it was adopted. Under an originalist approach, for example, if the Constitution did not prohibit capital punishment in 1789or in 1791, when the Bill of Rights was ratified, or in 1868, when the Fourteenth Amend- 


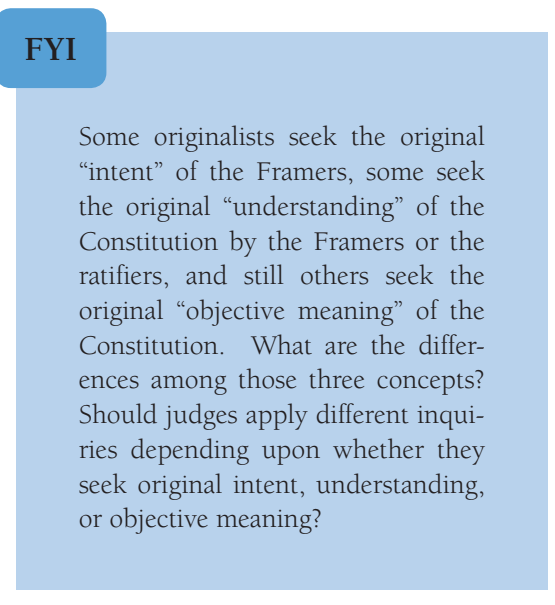

ment was ratified-then it does not prohibit it today. Conversely, under an originalist approach, if the Constitution in 1789-or, again, in 1791 or 1868 — did not authorize Congress to rely on its authority under the Commerce Clause to regulate certain matters, then arguably Congress lacks that power today, as well. (We will consider in Chapter 3 whether changes in the nature of "commerce" inevitably should lead to the conclusion that Congress has greater power to regulate local matters today than it did at the time of the Framing.)

Proponents of originalism generally offer three principal arguments in support of their approach to interpreting the Constitution. First, they argue that originalism is the only approach to constitutional interpretation that properly recognizes the Constitution's status as law. Second, they argue that originalism is necessary to preserve democratic values. Third, they argue that originalism is uniquely promising for constraining the ability of judges to impose their own personal views under the guise of constitutional interpretation. We discuss these claims in turn.

First, originalists generally argue that because we have always treated written law as having a fixed meaning-this is true, for example, for statutes, whose meaning does not generally evolve over time- the Constitution, which is a form of law, must have a fixed meaning, as well. And, originalists argue, just as a statute's meaning is determined by reference to the understandings of the people who enacted it, the meaning of a provision of the Constitution must also be determined by reference to the people who were responsible for its enactment. Originalists do not necessarily deny the existence of the dead-hand problem, but they argue that if the Constitution is authoritative only to the extent that we agree with it today, then it is not really law at all but instead is simply a makeweight.

Second, proponents of originalism argue that only their approach is consistent with the proper judicial role in a democratic society. Any approach other than originalism, they argue, inevitably seeks constitutional meaning in evolving or current values. But a democratic system, they argue, does not need constitutional guarantees to ensure that its laws reflect current values. And it is fundamentally anti-democratic, they contend, to permit unelected judges to invalidate democratically enacted laws that are not inconsistent with the original meaning of the Constitution.

Third, originalists contend that because judicial review is by its very nature countermajoritarian - a theme that we will explore in detail in Chapter 2-it is essential to ensure that judges employing it exercise, in Alexander Hamilton's words, "judgment" rather than "will," The Federalist No. 78 - that is, that judges simply interpret the law rather than make the law. Because the original meaning of the Constitution is fixed, originalists argue, it can 
be objectively determined by a judge without reference to his own political preferences. In contrast, they argue, if the Constitution's meaning "evolves," but derives from something other than democratic enactments, then the judges seeking the Constitution's meaning will not be restrained in their ability to impose their own personal views under the guise of constitutional interpretation.

The basic approach of originalism, of course, is not a new approach to interpretation. But it has attracted renewed attention and support in the last few decades, in part as a response to the perceived non-originalism of the Supreme Court in the 1950s and 1960s, an era that saw a substantial judicial expansion of rights subject to constitutional protection.

\section{Perspective and Analysis}

Justice Antonin Scalia has arguably been the most well-known and influential originalist. Consider his defense of the approach:

[O]riginalism seems to me more compatible with the nature and purpose of a Constitution in a democratic system. A democratic society does not, by and large, need constitutional guarantees to insure that its laws will reflect "current values." Elections take care of that quite well. The purpose of constitutional guarantees *** is precisely to prevent the law from reflecting certain changes in original values that the society adopting the Constitution thinks fundamentally undesirable.

He has also criticized the alternatives to originalism:

[T]he central practical defect of nonoriginalism is fundamental and irreparable: the impossibility of achieving any consensus on what, precisely, is to replace original meaning, once that is abandoned. *** [In contrast, originalism] establishes a historical criterion that is conceptually quite separate from the preferences of the judge himself.

Antonin Scalia, Originalism: The Lesser Evil, 57 U. Cin. L. Rev. 849, 862-64 $\underline{(1989)}$.

\section{Non-Originalism}

In contrast, non-originalists generally believe that the Constitution's meaning today is not always the meaning that it had when it was ratified. Non-originalists accordingly look to a range of sources in interpreting the Constitution. This is not to say that all non- 
originalists believe that the original meaning is irrelevant to constitutional interpretation; to the contrary, virtually all theories of constitutional interpretation accord significantand in some cases dispositive-weight to the original meaning. Non-originalists often conclude that the original meaning of a constitutional provision, expressed at a very high level of generality, provides guidance for ascertaining the relevant constitutional rule. Nonoriginalists, for example, might read the Equal Protection Clause of the Fourteenth Amendment to announce a general rule about equal treatment, which they might be willing to apply in a manner that the Framers of the Fourteenth Amendment did not contemplate, such as to discrimination on the basis of gender. But, for the reasons discussed below, non-originalists sometimes are willing to depart from the original meaning.

Non-originalists also often look to judicial precedent in seeking constitutional meaning. In this way, many non-originalists view constitutional interpretation as something akin to common-law decision-making, with constitutional principles evolving gradually over time. Non-originalists also often look to state practices to determine whether broad consensus has developed that a particular action or practice is acceptable or unacceptable.

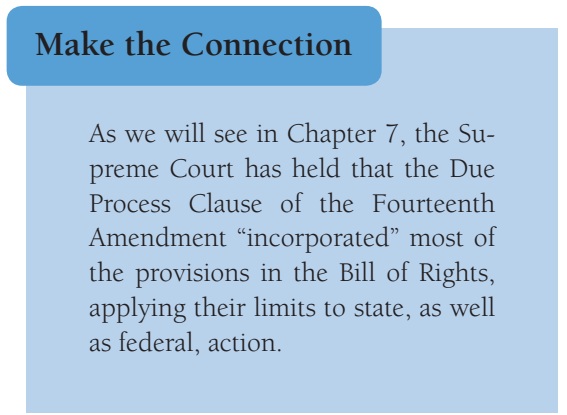
For example, if public flogging is banned in all states but one —and has been illegal in all states but one for decades-then a non-originalist might conclude that public flogging today is a "cruel and unusual" punishment within the meaning of the Eighth Amendment, even though it likely was not thought to be cruel and unusual in 1791, when the Eighth Amendment was ratified, or in 1868, when the Fourteenth Amendment was ratified.

Non-originalists are willing to depart from the original meaning of the Constitution because of concerns about originalism itself. Nonoriginalists have generally disputed originalists' claims that originalism is likely to produce determinate constitutional meaning today. More fundamentally, non-originalists argue that departure from the original meaning often is necessary to ensure that the Constitution retains legitimacy, which is essential if the public is to continue to accept the Constitution's binding character. We discuss these claims in turn.

First, non-originalists contend that most difficult constitutional questions that arise today cannot be answered by simple reference to the original understanding. They observe that the historical record is silent on many important provisions of the Constitution; that when the Framers did discuss the meaning of a particular provision of the Constitution, they often disagreed about its meaning; and that, in any event, the Framers did not contemplate, let alone discuss, most of the difficult questions that arise today or how the Constitution would apply to those problems. Accordingly, non-originalists argue that resort to the original meaning is unlikely to produce determinate constitutional meaning today. Non-originalists also argue that this likely indeterminism undermines the claim that originalism is uniquely promising as a way to constrain judges from imposing their own views under the guise of constitutional interpretation; faced with an ambiguous or 
indeterminate historical record, non-originalists contend, judges have discretion to choose the evidence of original meaning that best reflects their own personal values.

Second, and more important, non-originalists argue that originalism fails to account for the dead-hand problem, and thus risks producing a Constitution that fails the test of legitimacy. Originalism, non-originalists note, by definition gives voice to the values of the framing generation, and thus risks producing results that the American public today might find problematic. An originalist approach to the Fourteenth Amendment, for example, arguably would have required the Court to conclude, contrary to the decision in Brown v. Board of Education, 347 U.S. 483 (1954), that racial segregation in public schools does not violate the Equal Protection Clause. Similarly, discrimination on the basis of gender was commonplace in 1868 when the Fourteenth Amendment was ratified; a faithfully originalist approach almost certainly would permit the states today to prohibit women from (among other things) serving as lawyers, as they did at the time that the Amendment was ratified. See Bradwell v. Illinois, 83 U.S. (16 Wall.) 130 (1873).

Non-originalists recognize that the Constitution (in Article V) makes explicit provision for the adoption of amendments, but they argue that the potential of the amendment process to incorporate modern values into the Constitu-

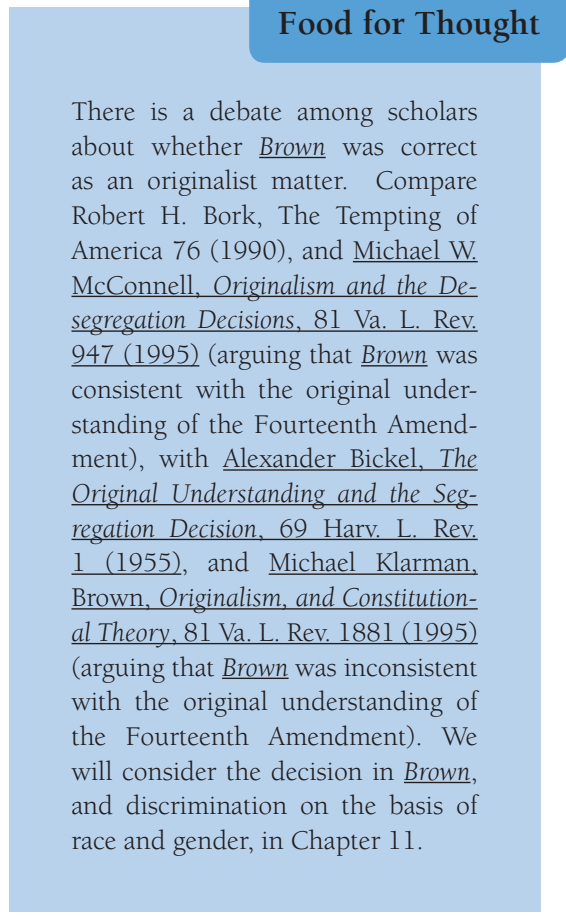
tion is illusory. In 1954, for example, when the Court decided Brown, Southern resistance would have prevented the ratification of an amendment prohibiting racial segregation. Similarly, as recently as the 1970s, a proposed amendment to prohibit discrimination on the basis of gender failed to achieve ratification in the required three-quarters of the States. These results, non-originalists contend, would substantially undermine respect for the Constitution, and thus the public's willingness to be bound by the Constitution. 


\section{Perspective and Analysis}

Justice William Brennan was an influential critic of originalism and a proponent of what some call "Living Constitutionalism." Consider his view:

A position that upholds constitutional claims only if they were within the specific contemplation of the Framers in effect establishes a presumption of resolving textual ambiguities against the claim of constitutional right. *** This is a choice no less political than any other; it expresses antipathy to claims of the minority to rights against the majority. $* * *$

Justice Brennan did not reject the notion that the Constitution has enduring principles, defined by the Framers' choices. But he disagreed with a formulaic application of those principles:

Current Justices read the Constitution in the only way that we can: as twentiethcentury Americans. We look to the history of the time of the framing and to the intervening history of interpretation. But the ultimate question must be: What do the words of the text mean in our time? For the genius of the Constitution rests not in any static meaning it might have had in a world that is dead and gone, but in the adaptability of its great principles to cope with current problems and current needs.

William J. Brennan, Jr., The Constitution of the United States: Contemporary Ratification, 27 S. Tex. L. Rev. 433, 436 (1986).

All of this discussion leads to an important question: Which interpretive method does the Supreme Court use? As you will see, this question has no simple answer. As you read the Court's cases, you will find three categories of decisions.

In some cases, especially older ones, the Supreme Court has insisted emphatically that only the original meaning of the Constitution matters. The decision in South Carolina v. United States, 199 U.S. 437 (1905), made this point with unmistakable clarity. The Court said: "The Constitution is a written instrument. As such its meaning does not alter. That which it meant when adopted, it means now." Id. at 448.

In other cases, however, the Court has squarely rejected the idea that it must follow the original meaning of the Constitution. For example, in Home Building \& Loan Assn. v. Blaisdell, 290 U.S. 398 (1934), which we will consider in Chapter 18, the Court allowed a state law to alter the obligation of a mortgage contract even though it recognized that this result likely conflicted with the original understanding of Contracts Clause in Article I, Section 10. The Court unapologetically rejected the idea that "the great clauses of the Constitution must be confined to the interpretation which the framers, with the conditions and outlook of their time, would have placed upon them." Id. at 443. 
Most modern cases fall somewhere in between these two extremes. Usually, the Court considers evidence of the original meaning without making broad pronouncements about whether the original meaning must control. Sometimes the Court follows the original meaning, and sometimes it does not. Interestingly, even though the current Justices have very different views on constitutional interpretation, they all cite the Federalist Papers, the records of the Constitutional Convention, and the state ratifying debates from time to time because they all consider these sources to be influential. In many cases, the Court tries to follow the original meaning, but simply cannot agree on what it was. For example, as we will see in Chapter 4 in U.S. Term Limits, Inc. v. Thornton, 514 U.S. 779 (1995), both the majority and dissenting opinions claim to follow the original meaning.

In reading the cases in this book, consider not only the results that the Supreme Court reaches, but also what interpretive methodology the Court is using.

This section has provided an overview of the competing approaches to constitutional interpretation. Although we directly address the arguments for originalism and nonoriginalism here, in many respects all of the material that follows in this book speaks, even if only indirectly, to the same question. Indeed, the judicial decisions in this book about what the Constitution means often are as much about the appropriate way to interpret the Constitution as they are about the meaning of the particular provisions at issue. As you read those materials, be sensitive to the relationship between arguments about the meaning of particular provisions of the Constitution and arguments about the appropriate way to interpret the Constitution more generally.

\section{An Illustrative Case}

As we have discussed, constitutional interpretation often involves the need to ascribe concrete meaning to ambiguous text and the need to apply that text to concrete circumstances. What considerations are relevant in engaging in those inquiries?

In the case that follows, the Court considered the constitutionality under the Second Amendment of the District of Columbia's prohibition on the possession of handguns in the home. As you read the three opinions in the case-one for the Court and two in dissent-consider the roles that text, history, structure, and policy play in the interpretations that the Justices advanced. 


\section{District of Columbia v. Heller}

554 U.S. $570(2008)$

Justice SCALIA delivered the opinion of the Court.

The District of Columbia generally prohibits the possession of handguns. It is a crime to carry an unregistered firearm, and the registration of handguns is prohibited. See D.C.Code $\$ \S 7-2501.01(12), 7-2502.01(a), 7-2502.02(a)(4)(2001)$. Wholly apart from that prohibition, no person may carry a handgun without a license, but the chief of police may issue licenses for 1-year periods. See $\$ \S 22-4504(a), 22-4506$. District of Columbia law also requires residents to keep their lawfully owned firearms, such as registered long guns, "unloaded and dissembled or bound by a trigger lock or similar device" unless they are located in a place of business or are being used for lawful recreational activities. See § 7-2507.02.

[Respondent Dick Heller, a D.C. special police officer authorized to carry a handgun while on duty at the Federal Judicial Center, wished to keep a handgun at home. He filed a lawsuit in District Court challenging these provisions under the Second Amendment. The District Court dismissed respondent's complaint, but the Court of Appeals directed the District Court to enter summary judgment for respondent.]

The Second Amendment provides: "A well regulated Militia, being necessary to the security of a free State, the right of the people to keep and bear Arms, shall not be infringed." *** The two sides in this case have set out very different interpretations of the Amendment. Petitioners and today's dissenting Justices believe that it protects only the right to possess and carry a firearm in connection with militia service. Respondent argues that it protects an individual right to possess a firearm unconnected with service in a militia, and to use that arm for traditionally lawful purposes, such as self-defense within the home.

The Second Amendment is naturally divided into two parts: its prefatory clause and its operative clause. ${ }^{* * *}$ Logic demands that there be a link between the stated purpose and the command. [That] requirement of logical connection may cause a prefatory clause to resolve an ambiguity in the operative clause ***. But apart from that clarifying function, a prefatory clause does not limit or expand the scope of the operative clause. Therefore, while we will begin our textual analysis with the operative clause, we will return to the prefatory clause to ensure that our reading of the operative clause is consistent with the announced purpose.

\section{Operative Clause}

"Right of the People." The first salient feature of the operative clause is that it codifies a "right of the people." The unamended Constitution and the Bill of Rights use the phrase "right of the people" two other times, in the First Amendment's Assembly-and-Petition Clause and in the Fourth Amendment's Search-and-Seizure Clause. The Ninth Amendment uses very similar terminology ***. All three of these instances unambiguously refer to individual rights, not "collective" rights, or rights that may be exercised only through participation in some corporate body. *** What is more, in all [other] provisions of the 
Constitution that mention "the people," the term unambiguously refers to all members of the political community, not an unspecified subset. [This] contrasts markedly with the phrase "the militia" in the prefatory clause. As we will describe below, the "militia" in colonial America consisted of a subset of "the people"-those who were male, able bodied, and within a certain age range. Reading the Second Amendment as protecting only the right to "keep and bear Arms" in an organized militia therefore fits poorly with the operative clause's description of the holder of that right as "the people." We start therefore with a strong presumption that the Second Amendment right is exercised individually and belongs to all Americans.

"Keep and bear Arms." We move now from the holder of the right— "the people"to the substance of the right: "to keep and bear Arms." Before addressing the verbs "keep" and "bear," we interpret their object: "Arms." The 18th-century meaning is no different from the meaning today. The 1773 edition of Samuel Johnson's dictionary defined "arms" as "weapons of offence, or armour of defence." 1 Dictionary of the English Language 107 (4th ed.) (hereinafter Johnson). *** The term was applied, then as now, to weapons that were not specifically designed for military use and were not employed in a military capacity.

We turn to the phrases "keep arms" and "bear arms." Johnson defined "keep" as, most relevantly, "[t]o retain; not to lose," and "[t]o have in custody." Johnson 1095. *** Thus, the most natural reading of "keep Arms" in the Second Amendment is to "have weapons."

At the time of the founding, as now, to "bear" meant to "carry." When used with "arms," however, the term has a meaning that refers to carrying for a particular purposeconfrontation. ** Although the phrase implies that the carrying of the weapon is for the purpose of "offensive or defensive action," it in no way connotes participation in a structured military organization. From our review of founding-era sources, we conclude that this natural meaning was also the meaning that "bear arms" had in the 18th century. In numerous instances, "bear arms" was unambiguously used to refer to the carrying of weapons outside of an organized militia. The most

\section{Food for Thought}

As the Court's discussion makes clear, some state constitutions explicitly extended the right to bear arms to self-defense. Does this fact support or undermine the Court's interpretation? prominent examples are those most relevant to the Second Amendment: Nine state constitutional provisions written in the 18th century or the first two decades of the 19th, which enshrined a right of citizens to "bear arms in defense of themselves and the state" or "bear arms in defense of himself and the state." It is clear from those formulations that "bear arms" did not refer only to carrying a weapon in an organized military unit.

Meaning of the Operative Clause. Putting all of these textual elements together, we find that they guarantee the individual right to possess and carry weapons in case of confrontation. This meaning is strongly confirmed by the historical background of the Second Amendment. *** Between the Restoration and the Glorious Revolution, the Stuart Kings Charles II and James II succeeded in using select militias loyal to them to suppress political 
dissidents, in part by disarming their opponents. These experiences caused Englishmen to be extremely wary of concentrated military forces run by the state and to be jealous of their arms. They accordingly obtained an assurance from William and Mary, in the Declaration of Right (which was codified as the English Bill of Rights), that Protestants would never be disarmed ${ }^{* * *}$. This right has long been understood to be the predecessor to our Second Amendment.

By the time of the founding, the right to have arms had become fundamental for English subjects. Blackstone [cited] the arms provision of the Bill of Rights as one of the fundamental rights of Englishmen, [describing it as] "the natural right of resistance and self-preservation" ***. Thus, the right secured in 1689 as a result of the Stuarts' abuses was by the time of the founding understood to be an individual right protecting against both public and private violence. And, of course, what the Stuarts had tried to do to their political enemies, George III had tried to do to the colonists.

There seems to us no doubt, on the basis of both text and history, that the Second Amendment conferred an individual right to keep and bear arms. [We now determine] whether the prefatory clause of the Second Amendment comports with our interpretation of the operative clause. $* * *$

\section{Relationship between Prefatory Clause and Operative Clause}

*** The debate with respect to the right to keep and bear arms, as with other guarantees in the Bill of Rights, was not over whether it was desirable (all agreed that it was) but over whether it needed to be codified in the Constitution. During the 1788 ratification debates, the fear that the federal government would disarm the people in order to impose rule through a standing army or select militia was pervasive in Antifederalist rhetoric. Federalists responded that because Congress was given no power to abridge the ancient right of individuals to keep and bear arms, such a force could never oppress the people. It was understood across the political spectrum that the right helped to secure the ideal of a citizen militia, which might be necessary to oppose an oppressive military force if the constitutional order broke down.

It is therefore entirely sensible that the Second Amendment's prefatory clause announces the purpose for which the right was codified: to prevent elimination of the militia. The prefatory clause does not suggest that preserving the militia was the only reason Americans valued the ancient right; most undoubtedly thought it even more important for self-defense and hunting. But the threat that the new Federal Government would destroy the citizens' militia by taking away their arms was the reason that right—unlike some other English rights—was codified in a written Constitution.

[P] etitioners' interpretation does not even achieve the narrower purpose that prompted codification of the right. If, as they believe, the Second Amendment right is no more than the right to keep and use weapons as a member of an organized militia, [then] it does not assure the existence of a "citizens' militia" as a safeguard against tyranny. For Congress retains plenary authority to organize the militia, which must include the authority to say 
who will belong to the organized force. ${ }^{* * *}$ Thus, if petitioners are correct, the Second Amendment protects citizens' right to use a gun in an organization from which Congress has plenary authority to exclude them. It guarantees a select militia of the sort the Stuart kings found useful, but not the people's militia that was the concern of the founding generation.

Our interpretation is confirmed by analogous arms-bearing rights in state constitutions that preceded and immediately followed adoption of the Second Amendment. Four States adopted analogues to the Federal Second Amendment in the period between independence and the ratification of the Bill of Rights. Two of them-Pennsylvania and Vermontclearly adopted individual rights unconnected to militia service. Pennsylvania's Declaration

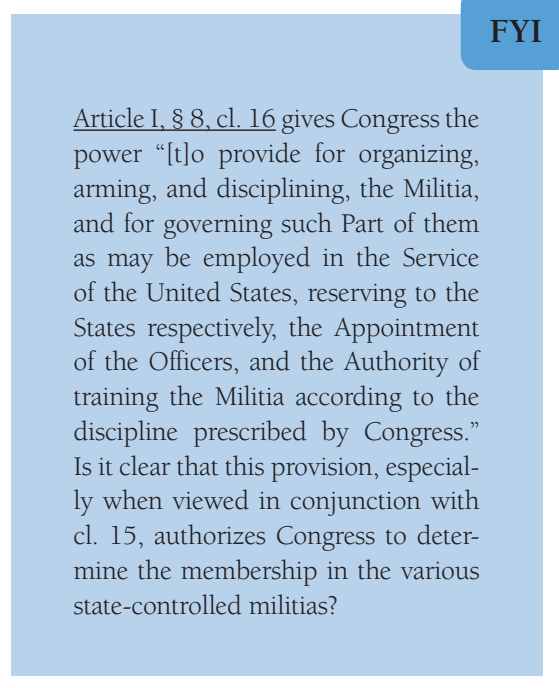
of Rights of 1776 said: "That the people have a right to bear arms for the defence of themselves, and the state ***." In 1777, Vermont adopted the identical provision, except for inconsequential differences in punctuation and capitalization. ${ }^{* *}$ North Carolina also codified a right to bear arms in 1776: "That the people have a right to bear arms, for the defence of the State ***." Declaration of Rights $\S$ XVII. This could plausibly be read to support only a right to bear arms in a militia-but that is a peculiar way to make the point in a constitution that elsewhere repeatedly mentions the militia explicitly. We [believe] that the most likely reading of [these] pre-Second Amendment state constitutional provisions is that they secured an individual right to bear arms for defensive purposes. [That] is strong evidence that that is how the founding generation conceived of the right.

Justice STEVENS places overwhelming reliance upon this Court's decision in United States v. Miller, 307 U.S. 174 (1939). [According to Justice STEVENS, Miller held that] the Second Amendment "protects the right to keep and bear arms for certain military purposes, but that it does not curtail the legislature's power to regulate the nonmilitary use and ownership of weapons." [But] Miller did not hold that and cannot possibly be read to have held that. The judgment in the case upheld against a Second Amendment challenge two men's federal convictions for transporting an unregistered short-barreled shotgun in interstate commerce, in violation of the National Firearms Act, 48 Stat. 1236. It is entirely clear that the Court's basis for saying that the Second Amendment did not apply was [that] the type of weapon at issue was not eligible for Second Amendment protection: "In the absence of any evidence tending to show that the possession or use of a [short-barreled shotgun] at this time has some reasonable relationship to the preservation or efficiency of a well regulated militia, we cannot say that the Second Amendment guarantees the right to keep and bear such an instrument." 307 U.S. at 178 (emphasis added). "Certainly," the Court continued, "it is not within judicial notice that this weapon is any part of the 
ordinary military equipment or that its use could contribute to the common defense." Ibid. $[\mathrm{H}]$ ad the Court believed that the Second Amendment protects only those serving in the militia, it would have been odd to examine the character of the weapon rather than simply note that the two crooks were not militiamen. ${ }^{* * *}$ We therefore read Miller to say only that the Second Amendment does not protect those weapons not typically possessed by law-abiding citizens for lawful purposes, such as short-barreled shotguns.

Like most rights, the right secured by the Second Amendment is not unlimited. *** Although we do not undertake an exhaustive historical analysis today of the full scope of the Second Amendment, nothing in our opinion should be taken to cast doubt on longstanding prohibitions on the possession of firearms by felons and the mentally ill, or laws forbidding the carrying of firearms in sensitive places such as schools and government buildings, or laws imposing conditions and qualifications on the commercial sale of arms. *** We also recognize another important limitation on the right to keep and carry arms. Miller said, as we have explained, that the sorts of weapons protected were those "in common use at the time." 307 U.S. at 179. We think that limitation is fairly supported by the historical tradition of prohibiting the carrying of "dangerous and unusual weapons."

We turn finally to the law at issue here. [As we have demonstrated,] the inherent right of self-defense has been central to the Second Amendment right. The handgun ban amounts to a prohibition of an entire class of "arms" that is overwhelmingly chosen by American society for that lawful purpose. The prohibition extends, moreover, to the home, where the need for defense of self, family, and property is most acute. Under any of the standards of scrutiny that we have applied to enumerated constitutional rights, banning from the home "the most preferred firearm in the nation to 'keep' and use for protection of one's home and family," $478 \mathrm{~F} .3 \mathrm{~d}$, at 400 , would fail constitutional muster. *** Whatever the reason, handguns are the most popular weapon chosen by Americans for self-defense in the home, and a complete prohibition of their use is invalid.

[T]he District's requirement (as applied to respondent's handgun) that firearms in the home be rendered and kept inoperable at all times [makes] it impossible for citizens to use them for the core lawful purpose of self-defense and is hence unconstitutional.

Before this Court petitioners have stated that "if the handgun ban is struck down and respondent registers a handgun, he could obtain a license, assuming he is not otherwise disqualified," by which they apparently mean if he is not a felon and is not insane. Respondent conceded at oral argument that he does not "have a problem [with] licensing" and that the District's law is permissible so long as it is "not enforced in an arbitrary and capricious manner." We therefore assume that petitioners' issuance of a license will satisfy respondent's prayer for relief and do not address the licensing requirement.

Justice BREYER [criticizes] us for declining to establish a level of scrutiny for evaluating Second Amendment restrictions. He proposes, explicitly at least, none of the traditionally expressed levels (strict scrutiny, intermediate scrutiny, rational basis), but rather a judge-empowering "interest-balancing inquiry" that "asks whether the statute burdens a protected interest in a way or to an extent that is out of proportion to the statute's salutary effects upon other important governmental interests." *** We know of no other enu- 
merated constitutional right whose core protection has been subjected to a freestanding "interest-balancing" approach. The very enumeration of the right takes out of the hands of government — even the Third Branch of Government - the power to decide on a case-bycase basis whether the right is really worth insisting upon. *** Constitutional rights are enshrined with the scope they were understood to have when the people adopted them, whether or not future legislatures or (yes) even future judges think that scope too broad. [The Second Amendment] is the very product of an interest-balancing by the peoplewhich Justice BREYER would now conduct for them anew. And whatever else it leaves to future evaluation, it surely elevates above all other interests the right of law-abiding, responsible citizens to use arms in defense of hearth and home.

We are aware of the problem of handgun violence in this country, and we take seriously the concerns raised by the many amici who believe that prohibition of handgun ownership is a solution. [But] the enshrinement of constitutional rights necessarily takes certain policy choices off the table. These include the absolute prohibition of handguns held and used for self-defense in the home. Undoubtedly some think that the Second Amendment is outmoded in a society where our standing army is the pride of our Nation, where well-trained police forces provide personal security, and where gun violence is a serious problem. That is perhaps debatable, but what is not debatable is that it is not the role of this Court to pronounce the Second Amendment extinct. [Affirmed.]

Justice STEVENS, with whom Justice SOUTER, Justice GINSBURG, and Justice BREYER join, dissenting.

The Second Amendment was adopted to protect the right of the people of each of the several States to maintain a well-regulated militia. It was a response to concerns raised during the ratification of the Constitution that the power of Congress to disarm the state militias and create a national standing army posed an intolerable threat to the sovereignty of the several States. Neither the text of the Amendment nor the arguments advanced by its proponents evidenced the slightest interest in limiting any legislature's authority to regulate private civilian uses of firearms. Specifically, there is no indication that the Framers of the Amendment intended to enshrine the common-law right of self-defense in the Constitution.

In 1934, Congress enacted the National Firearms Act, the first major federal firearms law. Upholding a conviction under that Act, this Court held that, "[i]n the absence of any evidence tending to show that possession or use of a 'shotgun having a barrel of less than eighteen inches in length' at this time has some reasonable relationship to the preservation or efficiency of a well regulated militia, we cannot say that the Second Amendment guarantees the right to keep and bear such an instrument." Miller, 307 U.S. at 178. The view of the Amendment we took in Miller — that it protects the right to keep and bear arms for certain military purposes, but that it does not curtail the Legislature's power to regulate the nonmilitary use and ownership of weapons-is both the most natural reading of the Amendment's text and the interpretation most faithful to the history of its adoption.

*** The preamble to the Second Amendment [is] comparable to provisions in several State Declarations of Rights that were adopted roughly contemporaneously with the Decla- 
ration of Independence. ${ }^{5}$ Those state provisions highlight the importance members of the founding generation attached to the maintenance of state militias; they also underscore the profound fear shared by many in that era of the dangers posed by standing armies. While the need for state militias has not been a matter of significant public interest for almost two centuries, that fact should not obscure the contemporary concerns that animated the Framers.

The parallels between the Second Amendment and these state declarations, and the Second Amendment's omission of any statement of purpose related to the right to use firearms for hunting or personal self-defense, is especially striking in light of the fact that the Declarations of Rights of Pennsylvania and Vermont did expressly protect such civilian uses at the time. Article XIII of Pennsylvania's 1776 Declaration of Rights announced that "the people have a right to bear arms for the defence of themselves and the state"; $\S 43$ of the Declaration assured that "the inhabitants of this state shall have the liberty to fowl and hunt in seasonable times on the lands they hold, and on all other lands therein not inclosed." And Article XV of the 1777 Vermont Declaration of Rights guaranteed "[t]hat the people have a right to bear arms for the defence of themselves and the State." The contrast between those two declarations and the Second Amendment reinforces the clear statement of purpose announced in the Amendment's preamble. It confirms that the Framers' single-minded focus in crafting the constitutional guarantee "to keep and bear arms" was on military uses of firearms, which they viewed in the context of service in state militias.

The preamble thus both sets forth the object of the Amendment and informs the meaning of the remainder of its text. ${ }^{* * *}$ The Court today tries to denigrate the importance of this clause of the Amendment by beginning its analysis with the Amendment's operative provision and returning to the preamble merely "to ensure that our reading of the operative clause is consistent with the announced purpose." That is not how this Court ordinarily reads such texts, and it is not how the preamble would have been viewed at the time the Amendment was adopted. *** Without identifying any language in the text that even mentions civilian uses of firearms, the Court proceeds to "find" its preferred reading in what is at best an ambiguous text, and then concludes that its reading is not foreclosed by the preamble. Perhaps the Court's approach to the text is acceptable advocacy, but it is surely an unusual approach for judges to follow.

[T] he words "the people" in the Second Amendment refer back to the object announced in the Amendment's preamble. They remind us that it is the collective action of individuals having a duty to serve in the militia that the text directly protects and, perhaps more importantly, that the ultimate purpose of the Amendment was to protect the States' share of the divided sovereignty created by the Constitution.

Although the Court's discussion of [the words "to keep and bear Arms"] treats them as two "phrases" —as if they read "to keep" and "to bear" — they describe a unitary right: to

${ }^{5}$ The Virginia Declaration of Rights II 13 (1776), provided: "That a well-regulated militia, composed of the body of the people, trained to arms, is the proper, natural, and safe defence of a free State; that Standing Armies, in time of peace, should be avoided, as dangerous to liberty; and that, in all cases, the military should be under strict subordination to, and governed by, the civil power." [Maryland, Delaware, and New Hampshire had similar provisions.] 
possess arms if needed for military purposes and to use them in conjunction with military activities. *** The term "bear arms" is a familiar idiom; when used unadorned by any additional words, its meaning is "to serve as a soldier, do military service, fight." 1 Oxford English Dictionary 634 (2d ed.1989). It is derived from the Latin arma ferre, which, translated literally, means "to bear [ferre] war equipment [arma]." *** Had the Framers wished to expand the meaning of the phrase "bear arms" to encompass civilian possession and use, they could have done so by the addition of phrases such as "for the defense of themselves," as was done in the Pennsylvania and Vermont Declarations of Rights. The unmodified use of "bear arms," by contrast, refers most naturally to a military purpose, as evidenced by its use in literally dozens of contemporary texts. *** When, as in this case, there is no [qualifier], the most natural meaning is the military one; and, in the absence of any qualifier, it is all the more appropriate to look to the preamble to confirm the natural meaning of the text.

The Amendment's use of the term "keep" in no way contradicts the military meaning conveyed by the phrase "bear arms" and the Amendment's preamble. To the contrary, a number of state militia laws in effect at the time of the Second Amendment's drafting used the term "keep" to describe the requirement that militia members store their arms at their homes, ready to be used for service when necessary. The Virginia military law, for example, ordered that "every one of the said officers, non-commissioned officers, and privates, shall constantly keep the aforesaid arms, accoutrements, and ammunition, ready to be produced whenever called for by his commanding officer." Act for Regulating and Disciplining the Militia, 1785 Va. Acts ch. 1, § 3, p. 2. "[K]eep and bear arms" thus perfectly describes the responsibilities of a framing-era militia member.

[T] he single right that [the clause describes] is both a duty and a right to have arms available and ready for military service, and to use them for military purposes when necessary. ${ }^{* * *}$ When each word in the text is given full effect, the Amendment is most naturally read to secure to the people a right to use and possess arms in conjunction with service in a well-regulated militia.

Two themes relevant to our current interpretive task ran through the debates on the original Constitution. "On the one hand, there was a widespread fear that a national standing Army posed an intolerable threat to individual liberty and to the sovereignty of the separate States." Perpich v. Department of Defense, 496 U.S. 334, 340 (1990). On the other hand, the Framers recognized the dangers inherent in relying on inadequately trained militia members "as the primary means of providing for the common defense," Perpich, 496 U.S. at $340 .{ }^{* * *}$ In order to respond to those twin concerns, a compromise was reached: Congress would be authorized to raise and support a national Army and Navy, and also to organize, arm, discipline, and provide for the calling forth of "the Militia." U.S. Const., Art. I, \& 8, cls. 12-16. The President, at the same time, was empowered as the "Commander in Chief of the Army and Navy of the United States, and of the Militia of the several States, when called into the actual Service of the United States." Art. II, \& 2. But, with respect to the militia, a significant reservation was made to the States: [the] States respectively 
would retain the right to appoint the officers and to train the militia in accordance with the discipline prescribed by Congress. Art. I, \& 8, cl. 16. ${ }^{20}$

But the original Constitution's retention of the militia and its creation of divided authority over that body did not prove sufficient to allay fears about the dangers posed by a standing army [because] it did not prevent Congress from providing for the militia's disarmament. $* * *$ This sentiment was echoed at a number of state ratification conventions; indeed, it was one of the primary objections to the original Constitution voiced by its opponents.

[In response, upon ratifying the Constitution several states proposed amendments to the document.] The relevant proposals sent by the Virginia Ratifying Convention read as follows:

"17th, That the people have a right to keep and bear arms; that a well regulated Militia composed of the body of the people trained to arms is the proper, natural and safe defence of a free State. That standing armies are dangerous to liberty, and therefore ought to be avoided, as far as the circumstances and protection of the Community will admit; and that in all cases the military should be under strict subordination to and be governed by the civil power."

"19th. That any person religiously scrupulous of bearing arms ought to be exempted, upon payment of an equivalent to employ another to bear arms in his stead."

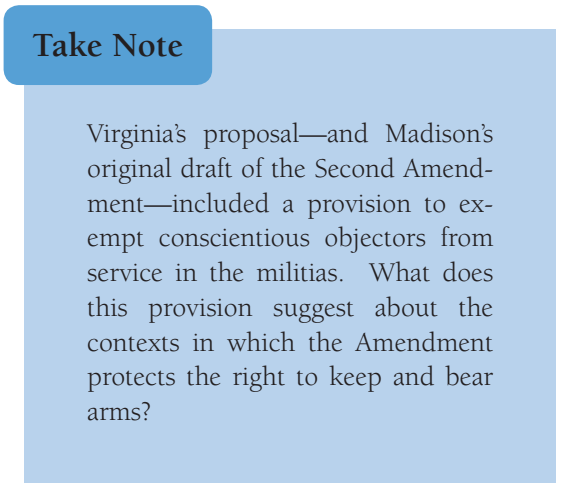

North Carolina adopted Virginia's proposals and sent them to Congress as its own ***. New York produced a proposal with nearly identical language. Notably, each of these proposals used the phrase "keep and bear arms" [and] embedded the phrase within a group of principles that are distinctly military in meaning.

By contrast, New Hampshire's proposal [described] the protection involved in more clearly personal terms[:] "Congress shall never disarm any Citizen unless such as are or have been in Actual Rebellion."

[James] Madison, charged with the task of assembling the proposals for amendments sent by the ratifying States, was the principal draftsman of the Second Amendment. [His] decision to model the Second Amendment on the distinctly military Virginia proposal is therefore revealing, since it is clear that he considered and rejected formulations that would have unambiguously protected civilian uses of firearms. When [his draft proposal] was debated and modified, it is reasonable to assume that all participants in the drafting process

${ }^{20}$ The Court assumes_-incorrectly, in my view — that even when a state militia was not called into service, Congress would have had the power to exclude individuals from enlistment in that state militia. That assumption is not supported by the text of the Militia Clauses of the original Constitution, which confer upon Congress the power to "organiz[e], ar[m], and disciplin[e], the Militia," Art. I. \& 8, cl. 16, but not the power to say who will be members of a state militia. It is also flatly inconsistent with the Second Amendment. *** 
were fully aware of the other formulations that would have protected civilian use and possession of weapons and that their choice to craft the Amendment as they did represented a rejection of those alternative formulations.

Until today, it has been understood that legislatures may regulate the civilian use and misuse of firearms so long as they do not interfere with the preservation of a well-regulated militia. The Court's announcement of a new constitutional right to own and use firearms for private purposes upsets that settled understanding ${ }^{* * *}$.

Justice BREYER, with whom Justice STEVENS, Justice SOUTER, and Justice GINSBURG join, dissenting.

[T] he protection the [Second] Amendment provides is not absolute. The Amendment permits government to regulate the interests that it serves. Thus, irrespective of what those interests are-whether they do or do not include an independent interest in self-defensethe majority's view cannot be correct unless it can show that the District's regulation is unreasonable or inappropriate in Second Amendment terms. This the majority cannot do.

The majority is wrong when it says that the District's law is unconstitutional "[u]nder any of the standards of scrutiny that we have applied to enumerated constitutional rights." [It] certainly would not be unconstitutional under, for example, a "rational basis" standard, which requires a court to uphold regulation so long as it bears a "rational relationship" to a "legitimate governmental purpose." Heller v. Doe, 509 U.S. 312, 320 (1993). The law at issue here, which in part seeks to prevent gun-related accidents, at least bears a "rational relationship" to that "legitimate" life-saving objective.

Respondent proposes that the Court adopt a "strict scrutiny" test, which would require reviewing with care each gun law to determine whether it is "narrowly tailored to achieve a compelling governmental interest." Abrams v. Johnson, 521 U.S. 74, 82 (1997). But the majority implicitly, and appropriately, rejects that suggestion by broadly approving a set of laws [whose] constitutionality under a strict scrutiny standard would be far from clear. Indeed, [almost] every gun-control regulation will seek to advance (as the one here does) a "primary concern of every government-a concern for the safety and indeed the lives of its citizens." United States v. Salerno, 481 U.S. 739, 755 (1987). *** Thus, any attempt in theory to apply strict scrutiny to gun regulations will in practice turn into an interestbalancing inquiry, with the interests protected by the Second Amendment on one side and the governmental public-safety concerns on the other, the only question being whether the regulation at issue impermissibly burdens the former in the course of advancing the latter.

I would simply adopt such an interest-balancing inquiry explicitly. *** "[W]here a law significantly implicates competing constitutionally protected interests in complex ways," the Court generally asks whether the statute burdens a protected interest in a way or to an extent that is out of proportion to the statute's salutary effects upon other important governmental interests. See Nixon v. Shrink Missouri Government PAC, 528 U.S. 377, 402 (2000) (BREYER, J., concurring). Any answer would take account both of the statute's effects upon the competing interests and the existence of any clearly superior less restrictive alternative. 
The only dispute regarding [the trigger-lock] provision appears to be whether the Constitution requires an exception that would allow someone to render a firearm operational when necessary for self-defense ***. The District concedes that such an exception exists. *** And because I see nothing in the District law that would preclude the existence of a background common-law self-defense exception, I would avoid the constitutional question by interpreting the statute to include it. See Ashwander v. TVA, 297 U.S. 288, 348 (1936) (Brandeis, J., concurring).

No one doubts the constitutional importance of [the] basic objective [of the District's ban on handgun possession], saving lives. But there is considerable debate about whether the [provision] helps to achieve that objective. $* * *$ Petitioners, and their amici, have presented us with [statistics about handgun violence.] *** From 1993 to 1997, there were 180,533 firearm-related deaths in the United States, an average of over 36,000 per year. *** In over one in every eight firearm-related deaths in 1997, the victim was someone under the age of 20. ${ }^{* * *}$ From 1993 to 1997, 81\% of firearm-homicide victims were killed by handgun. *** Handguns also appear to be a very popular weapon among criminals. ${ }^{* * *}$ Statistics further suggest that urban areas, such as the District, have different experiences with gun-related death, injury, and crime, than do less densely populated rural areas. A disproportionate amount of violent and property crimes occur in urban areas, and urban criminals are more likely than other offenders to use a firearm during the commission of a violent crime.

Respondent and his many amici [disagree] strongly with the District's predictive judgment that a ban on handguns will help solve the crime and accident problems that those figures disclose. ${ }^{* * *}$ First, they point out that, since the ban took effect [in 1976], violent crime in the District has increased, not decreased. $* * *$ Second, respondent's amici point to a statistical analysis that [concludes] that strict gun laws are correlated with more murders, not fewer. $* * *$ Third, they point to evidence indicating that firearm ownership does have a beneficial self-defense effect. ${ }^{* * *}$ Fourth, respondent's amici argue that laws criminalizing gun possession are self-defeating, as evidence suggests that they will have the effect only of restricting law-abiding citizens, but not criminals, from acquiring guns.

[T] he District and its amici [respond] with studies of their own. *** The upshot is a set of studies and counterstudies that, at most, could leave a judge uncertain about the proper policy conclusion. [But] legislators, not judges, have primary responsibility for drawing policy conclusions from empirical fact. [D]eference to legislative judgment seems particularly appropriate here, where the judgment has been made by a local legislature, with particular knowledge of local problems and insight into appropriate local solutions. *** For these reasons, I conclude that the District's statute properly seeks to further the sort of life-preserving and public-safety interests that the Court has called "compelling."

The District's statute burdens the Amendment's first and primary objective hardly at all. [T] here is general agreement among the Members of the Court that the principal (if not the only) purpose of the Second Amendment is found in the Amendment's text: the preservation of a "well regulated Militia." *** To begin with, the present case has nothing 
to do with actual military service. [And] the District's law does not seriously affect military training interests. The law permits residents to engage in activities that will increase their familiarity with firearms. They may register (and thus possess in their homes) weapons other than handguns, such as rifles and shotguns. ${ }^{* *}$ And while the District law prevents citizens from training with handguns within the District, [the] adjacent States do permit the use of handguns for target practice, and those States are only a brief subway ride away. [G]iven the costs already associated with gun ownership and firearms training, I cannot say that a subway ticket and a short subway ride (and storage costs) create more than a minimal burden.

The District's law does prevent a resident from keeping a loaded handgun in his home. And it consequently makes it more difficult for the householder to use the handgun for self-defense in the home against intruders, such as burglars. [But] there is no clearly superior, less restrictive alternative to the District's handgun ban [because] the ban's very objective is to reduce significantly the number of handguns in the District ***. [A]ny measure less restrictive in respect to the use of handguns for self-defense will, to that same extent, prove less effective in preventing the use of handguns for illicit purposes. If a resident has a handgun in the home that he can use for self-defense, then he has a handgun in the home that he can use to commit suicide or engage in acts of domestic violence. ${ }^{* * *}$ $[T]$ he District law is tailored to the life-threatening problems it attempts to address. The law concerns one class of weapons, handguns, leaving residents free to possess shotguns and rifles, along with ammunition. The area that falls within its scope is totally urban. ***

The majority derides my approach as "judge-empowering." I take this criticism seriously, but I do not think it accurate. *** Application of such an approach, of course, requires judgment, but the very nature of the approach-requiring careful identification of the relevant interests and evaluating the law's effect upon them-limits the judge's choices; and the method's necessary transparency lays bare the judge's reasoning for all to see and to criticize.

The majority's methodology is, in my view, substantially less transparent than mine. *** "Putting all of [the Second Amendment's] textual elements together," the majority says, "we find that they guarantee the individual right to possess and carry weapons in case of confrontation." Then, three pages later, it says that "we do not read the Second Amendment to permit citizens to carry arms for any sort of confrontation." Yet, with one critical exception, it does not explain which confrontations count. It simply leaves that question unanswered.

Nor is it at all clear to me how the majority decides which loaded "arms" a homeowner may keep. The majority says that that Amendment protects those weapons "typically possessed by law-abiding citizens for lawful purposes." This definition conveniently excludes machineguns, but permits handguns, which the majority describes as "the most popular weapon chosen by Americans for self-defense in the home." But what sense does this approach make? According to the majority's reasoning, if Congress and the States lift restrictions on the possession and use of machineguns, and people buy machineguns to protect their homes, the Court will have to reverse course and find that the Second Amend- 
ment does, in fact, protect the individual self-defense-related right to possess a machinegun. On the majority's reasoning, if tomorrow someone invents a particularly useful, highly dangerous self-defense weapon, Congress and the States had better ban it immediately, for once it becomes popular Congress will no longer possess the constitutional authority to do so. *** There is no basis for believing that the Framers intended such circular reasoning.

I am similarly puzzled by the majority's list [of] provisions that in its view would survive Second Amendment scrutiny. *** Why these? Is it that similar restrictions existed in the late 18th century? The majority fails to cite any colonial analogues. ${ }^{* * *}$

The argument about method, however, is by far the less important argument surrounding today's decision. Far more important are the unfortunate consequences that today's decision is likely to spawn. Not least of these [is] the fact that the decision threatens to throw into doubt the constitutionality of gun laws throughout the United States. I can find no sound legal basis for launching the courts on so formidable and potentially dangerous a mission. In my view, there simply is no untouchable constitutional right guaranteed by the Second Amendment to keep loaded handguns in the house in crime-ridden urban areas.

\section{Points for Discussion}

\section{a. The Judicial Role and the Counter-Majoritarian Difficulty}

In holding that the challenged regulations were unconstitutional, the Court noted that "the enshrinement of constitutional rights necessarily takes certain policy choices off the table." Notice that in making this statement, the Court is also asserting its authority both to determine the meaning of the Constitution and to invalidate democratically enacted laws. Is it problematic to permit the Court to act in such a counter-majoritarian, and arguably anti-democratic, fashion? Or is it essential that the Court do so? This question will be the subtext of all the material that follows, but it will be the particular focus of Chapter 2 .

\section{b. Structural and Institutional Arrangements or Individual Rights?}

Justice Scalia concluded that the Second Amendment protects an individual right to keep and bear arms for purposes unrelated to participation in a state militia. Justice Stevens, by contrast, would have held that the Amendment is principally a structural protection to ensure that Congress cannot disarm the state militias. Which view do you find more convincing?

Notice that Justice Scalia did not deny the structural aims of the Second Amendment, and that Justice Stevens did deny that its structural aims advance the interest in liberty. The first several Parts of this book will focus on the principal structural and institutional arrangements that the Constitution creates, federalism and separation of powers. As we will see in detail, the Framers clearly viewed these arrangements as essential to preserving individual liberty. The remainder of the book will focus on the Constitution's explicit and

direct protections for individual rights. Does it make sense to treat structural provisions and rights provisions as separate and discrete features of the constitutional scheme? 


\section{c. Interpretive Methodology: Originalism v. Non-Originalism}

In interpreting the Second Amendment, Justice Scalia sought to determine its meaning at the time of the Framing. He relied on contemporaneous sources, such as eighteenthcentury dictionaries and state constitutional provisions. Justice Breyer focused on the competing state and individual interests implicated by the challenged regulation. Was his approach non-originalist? Or did he simply seek to apply the original meaning of the Second Amendment to modern circumstances? Which approach did you find more sensible or convincing?

\section{d. Interpretive Methodology: Originalism v. Originalism}

Like Justice Scalia, Justice Stevens took an originalist approach to the Second Amendment, but he reached a very different conclusion about the Amendment's meaning. To the extent that the two Justices relied on similar materials, whose arguments did you find more convincing? What does the fact that they relied in part on competing sources say about the viability of originalism as an approach to interpreting the Constitution?

Did their inquiries have the same objective? Notice that Justice Scalia focused principally on what the language of the Second Amendment likely would have meant at the time of its ratification. Justice Stevens spent considerable time addressing the Amendment's drafting history. Does that mean that Justice Stevens was attempting to discern Madison's-and the other Framers'-intent in ratifying the Amendment? Is there a difference between the original "objective meaning" and the original "intent"? Is there a difference in the evidence that one might use to establish meaning and intent?

Notice also that Justice Scalia read the individual phrases in the Second Amendment atomistically, asking what each phrase means before assembling those individual meanings into one, broader meaning. Justice Stevens, by contrast, read the Amendment more holistically, with a particular emphasis on what the preamble suggests about the meaning of what Justice Scalia referred to as the "operative" clause. Given that many of the Constitution's most important provisions are written at a high level of generality and often are ambiguous in their application, what is the role of text in constitutional interpretation? Whose approach in Heller to the text did you find most convincing?

\section{e. Level of Scrutiny}

As the competing opinions suggested, the Court often assesses the constitutionality of government action by evaluating the governmental interests advanced by the action and the burden that the action imposes on the constitutional right at issue. For example, the Court has never interpreted the Equal Protection Clause of the Fourteenth Amendment absolutely to prohibit the government from treating different classes of citizens differently. Some classifications_-such as a law providing that only persons over sixteen years of age are eligible to obtain driver's licenses-do not seem problematic, and accordingly are subjected only to "rational basis review," under which they are upheld as long as the classification is reasonably related to some legitimate governmental interest. Other classificationssuch as laws that deny government benefits on the basis of race-are deeply suspect, and 


\section{Make the Connection}

We discuss levels of scrutiny in Chapter 10, and in Chapters 8, 11, 12 , and 14 we consider their application in detail.

accordingly are subjected to "strict scrutiny," under which they can be upheld only if they are narrowly tailored to achieve a compelling governmental interest. Still other classificationssuch as those that distinguish on the basis of gender-are subjected to "intermediate scrutiny," which falls somewhere between rational basis review and strict scrutiny. The various levels of scrutiny generally reflect a judgment that few constitutional prohibitions are absolute, and that some government actions that burden protected rights nevertheless are defensible.

What level of scrutiny did the majority apply to the challenged regulations? Did the majority consider the government's interest in the challenged regulations? Or did the majority simply suggest that regulations that would have been prohibited in 1791 are prohibited today? Does the majority's suggestion that many regulations of the right to bear arms would be constitutional shed any light on this question? What level of scrutiny did Justice Stevens apply?

Justice Breyer explicitly proposed a test under which the challenged regulation's constitutionality turns on whether the statute "burdens a protected interest in a way or to an extent that is out of proportion to the statute's salutary effects upon other important governmental interests." Is this approach preferable to the majority's apparent approach of recognizing categories of absolutely forbidden regulations and of clearly permissible regulations?

\section{f. Role of Precedent}

Before $\underline{\text { Heller }}$, the Court had decided very few cases that even circumspectly interpreted the Second Amendment. Perhaps the most important was United States v. Miller, 307 U.S. 174 (1939), a brief opinion whose reasoning, as the competing approaches in $\underline{\text { Heller }}$ show, was far from clear. What is the role of precedent in interpreting the Constitution? Suppose that the Court in Miller had clearly held that the Second Amendment protects the right to keep and bear arms only in conjunction with participation in a state militia. Would the Court in Heller have been bound by that holding if it concluded that the Court in Miller had failed properly to discern the original meaning of the Amendment? When is it appropriate for the Court to overrule prior decisions that interpreted the Constitution? In answering this question, consider what remedies exist when the Court "errs" in interpreting the Constitution.

\section{g. The Constitution and Ambiguity}

The issue in $\underline{\text { Heller }}$ was both of great importance and seemingly fundamental. Why do you think it took the Court over 200 years squarely to resolve that issue? In fact, as we will see throughout this book, a surprising number of important constitutional questions have never been addressed by the Court. As you read the materials that follow, consider why, and whether constitutional ambiguity is a good or bad thing. 


\section{h. Role of the Dissenting Opinions}

Why did Justices Breyer and Stevens write such extensive dissenting opinions? Dissenting opinions do not carry any precedential value, as they represent the views of a minority of Justices. Are dissenting opinions simply an expression of disagreement for disagreement's sake? Are they templates for criticism of the Court's decision? If so, to what end? Are they in effect intended as the groundwork for a future change of course on the Court? In this book, we will regularly see dissenting opinions. If nothing else, does the Court's frequent inability to achieve unanimity suggest anything about the nature of constitutional interpretation? 


\section{Point-Counterpoint}

\section{Does Heller represent originalism's triumph or its failure?}

Point: Gregory E. Maggs

"Originalism" is a doctrine saying that judges should interpret the Constitution according to its original meaning. Proponents of originalism disagree on some points, such as whether judges should focus on the original intent of the Framers, the original understanding of the ratifiers, or the original objective meaning of the Constitution's text. But they all agree that none of these meanings change over time and that judges should not allow current policy considerations to affect their interpretation of the Constitution.

One common objection to originalism is that it does not produce certain results. The argument supporting this objection is that the text of the Constitution and the relevant historical materials are often too sparse or inconclusive to produce definitive answers to current constitutional issues. This deficiency may prevent judges from determining answers to important constitutional questions. Worse, it may allow judges to decide cases according to their own political preferences and then cover up what they are doing with make-weight arguments resting on vague historical documents.

At first glance, the Supreme Court's decision in District of Columbia v. Heller, 128 S. Ct. 2783 (2008), might appear to support this objection to originalism. Justice Scalia's opinion for the Court and Justice Stevens's dissenting opinion both claim to use originalist methodology, but they reach different conclusions. This result may suggest to some that originalism cannot answer the question of what the Second Amendment means. In addition, the Court's conservatives concluded that the gun control law at issue was unconstitutional, while the Court's liberals concluded that it was not. Because conservative politicians tend to oppose gun control, and liberal politicians tend to favor it, this division of the Justices might suggest that politics determined the Justices' positions.

But further reflection should reveal that Heller does not represent a failure of originalism. Any method of constitutional interpretation may produce differing conclusions. But originalism appears to be generally more determinate than other interpretative methods. Most of the cases in this book contain both majority and dissenting opinions, yet in very few of these cases did both sides attempt to use originalist methodology. Heller is an example, as is U.S. Term Limits v. Thornton, 514 U.S. 779 (1995), but not many others come to mind. Has any other method of constitutional interpretation produced comparable certainty?

As for policy preferences, a closer look casts doubt on suspicions that either the majority or the dissent in $\underline{\text { Heller }}$ was just voting for the outcome that it favored as a matter of policy. The District of Columbia statute was among the most extreme gun control laws in the nation. Even without knowing what the individual Justices actually think about gun 
control, it is difficult to believe that all of the members of the dissent, who generally favor personal rights, would want laws that effectively ban all handgun ownership. The majority meanwhile went out of its way to make clear, in dicta, that many kinds of gun regulations are still constitutional. Again, it is hard to imagine that all of the Justices in the majority would favor every possible regulation. Instead, $\underline{\text { Heller }}$ appears to represent a good faith effort to determine what the Second Amendment originally meant, and is thus a triumph of originalism.

Counterpoint: Peter J. Smith

Originalism's proponents contend that it is the only legitimate approach to constitutional interpretation because (they say) it is the only approach that accords to the Constitution an objectively identifiable fixed meaning and, in so doing, prevents the Justices from imposing their personal policy preferences under the guise of constitutional interpretation. Yet the dueling opinions in $\underline{\text { Heller }}$ demonstrate why originalism fails to live up to its promise.

The 27 words of the Second Amendment provoked over 100 pages of interpretive analysis, and led two incredibly intelligent, historically well-versed, and widely respected Justices to diametrically different interpretations. And this should not be surprising. Most constitutional provisions are written at a very high level of generality—such as "Equal Protection" or "Due Process" - that give few hints about their "original" meaning as applied to concrete circumstances. And even those-such as the Second Amendment—that seem to speak at a higher level of specificity often are susceptible to multiple (and irreconcilable) interpretations. (The language of the Amendment, with its prefatory and operative clauses, is particularly obscure.) Add to these textual ambiguities the historical ambiguities that originalism invites-Would a "reasonable person" in 1791 have understood the first half of the Second Amendment to qualify the second half? Even assuming we can find enough evidence of such understanding, what if reasonable people in 1791 (like reasonable people today) disagreed about the meaning of the Amendment?_and originalism rarely produces meaning any more determinate than any other approach to constitutional interpretation.

If originalism is unlikely (at least in difficult cases, which, after all, are the only ones that end up seeing the light of day in a courtroom) to produce determinate meaning, then originalists' claims about how it constrains judges begin to fall apart. A judge seeking the original meaning of the Second Amendment can focus either on early militia provisions in state Declarations of Rights (as did Justice Stevens) or instead on the even-earlier English Bill of Rights or the writings of Blackstone (as did Justice Scalia); on contemporaneous dictionary definitions (as did Justice Scalia) or instead on contemporaneous state militia laws that used similar words (as did Justice Stevens). A judge can read early state constitutional provisions referring explicitly to the right to bear arms for self-defense either to confirm (as did Justice Scalia) or to refute (as did Justice Stevens) the view that the Second Amendment similarly protects such a right. And although there is no obvious reason to doubt the sincerity with which the Justices approached the question, one cannot ignore that the Justices widely viewed as the most conservative sided with the view preferred by political 
conservatives and that the more liberal Justices sided with the view preferred by political liberals. Originalism, it seems, is not nearly as constraining as its proponents claim.

Legal scholars have long debated the original meaning of the Second Amendment. But historians recognize that any such question is not susceptible to one authoritative answer; the Constitution, after all, was ratified by collective decision-making (in each state, no less), which reflected a dizzying array of (often dueling) intentions, expectations, hopes, and fears. One can debate whether the majority or the dissent had the better of the arguments. But it is time that we stopped pretending that this particular approach to constitutional interpretation is any better than others at establishing rules for judges to do what they must: to exercise judgment. 